Portland State University

PDXScholar

$5-1972$

\title{
Illusion und Verfremdung: Begriff, Form und Wirkung; Bezogen auf Bertolt Brecht: "Der gute Mensch von Sezuan,"
}

Robert E. Folk

Portland State University

Follow this and additional works at: https://pdxscholar.library.pdx.edu/open_access_etds

Part of the German Literature Commons, and the Other German Language and Literature Commons Let us know how access to this document benefits you.

Recommended Citation

Folk, Robert E., "Illusion und Verfremdung: Begriff, Form und Wirkung; Bezogen auf Bertolt Brecht: "Der gute Mensch von Sezuan,"' (1972). Dissertations and Theses. Paper 953.

https://doi.org/10.15760/etd.953

This Thesis is brought to you for free and open access. It has been accepted for inclusion in Dissertations and Theses by an authorized administrator of PDXScholar. Please contact us if we can make this document more accessible: pdxscholar@pdx.edu. 
ILLUSION UND VERFREMDUNG, BEGRIFF, FORM UND WIRXUNG

Bezogen auf Bertolt Brecht,

"Der gute Mensoh von Sezuan"

von

BOBEBT E. FOLK

A thesis submitted in partial fulfiliment of the requirements for the degree of

MASTER OF ARTS

in

GERMAN

Portland State University

1972 
TO THE OFFICE OF GRADUATE STUDIES :

The members of the Comittee approve the thesia of Robert E. Folk presented May 10, 1972.

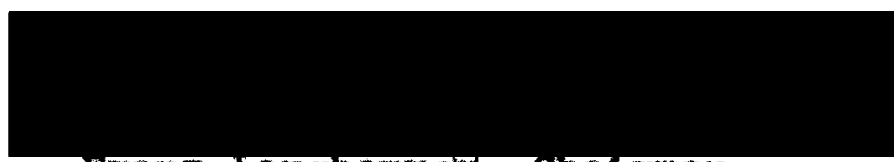

Franz Langhamet, chaiman

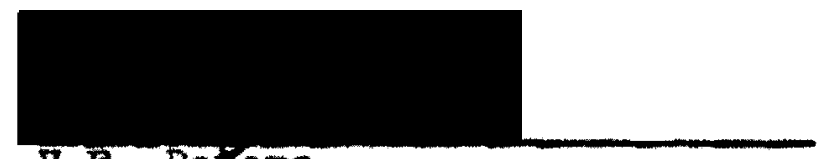

B.F. PeCers

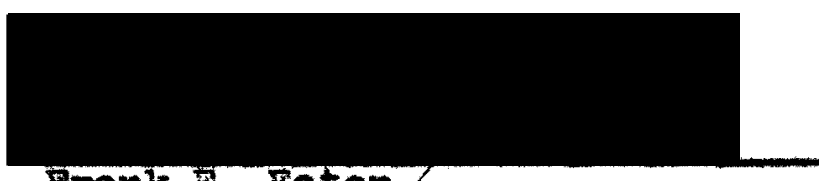

Frank Faton?

APPROVED,

a. Carol Healy, Chalman, Dppartment of Forelgn Languages Darid T. Clark, Dean of Graduate Studies

May 10, 1972 


\section{VORWORT}

Diese vorliegende Untersuchung geht von einer ganzheltilchen Tendenz in Hinblick auf die Behandlung der Brechtsohen Verstehenswelse von Illusion und Verfremdung aus, nlmlich devon, nicht nur die Begriffe Illusion und Verfremdung auf ihre ihnen innenwohnenden Strukturen hin zu bestimmen, sondern auch belde aufelnander zu bezlehen, und zwar so , dab elne gegenseitige Zuordnung zur Geltung gebracht werden soll, denn Brechts dichterische Konfrontation mit der Illusion 1st nur hinsichtilch des Gegenbegriffs der IIlusion, der Verfremdung, zu verstehen. Dlese Gegenberegung von Illusion und Verfremdung soll nach Brecht dem Zweck des Theaters dienen, namlioh der Aufdeckung der W1dersprtohe in dem kap1talistischen System, weshalb Brecht auch das Theater das "dialektisohe" nennt. Es handelt sioh um die Gegenberegung, die dem Zusohauer ine kritische Haltung den abgebildeten geselischaftichen Vorgungen (auch letztlich den elgenen gegentber) ermoglichen soll.

Dieser ganzhe1tilchen Tendenz ontsprechend w1rd rersucht, ron den grundlegenden begrifflichen und theoretischen Wurzeln der ganzen zun Thema gemachton Problematik her die real1sierten Intentionen in der Brechtsohen "Praris" darrustellen. Deshalb gliedert sich diese Arbelt in drel Telle: 1) Illusion, 2) Verfremdung und 3) Darstellung und Konkre- 
tion des Hauptmittels der Brechtsohen Verfremdung, d.1. die Sprache.

1. Te1l, Es stellt alch Innerhalb der Untersuchung heraus, daB belde Begriffe (IIlusion und Verfrendung) auf eine fundamentale Struktur hin durchsichtis gemacht werden konnen, die im Ausgang ron der Behandlung der Illusion konstrulert w1rd. Bel der Erarbeltung dieses Begriffs tritt zutage, daB kein Autor elne zufriedenstellende Definition anzubleten vermag, auch nioht Brecht selber, der sich lelder nlcht "exakt-syatemat1sch" aber die Illusion gelubert hat, deshalb 1st es fur mlch erforderlich, diesen Begriff Induktiv, d.h. anhand zweler ausgewhlter Theorlen, zu formieren. D1eser Illusionsbegriff wird dann welter auf die Brechtsoho Aurfasaung von Illusion abertragen, das bedeutet, d1. Auffassung Brechts von Illusion wird in inn integriert. Dieses wird dadurch erbracht, daB der erarbeltete Begriff mod1flzlert wird, und zwar derart, daB die Illusion sich in zwel Arten aufspaltet. M1t dieser Fassung wird es sodann mog11ch seln, sowohl Brechts Akzeptieren als auch selne Ablohnung der Illusion elnzufassen und deutlich zu machen.

2. Te11, Was die begriffllohe Fassung der Verfremdung anbelangt, so werden die von Brecht zahlreloh und zerstreut gegebenen Definitionen aurgenommen. Es wind sodann der Versuch gemacht, die Verfremdung, so wie sie im Dienste der Brechtschen Intention steht, als ProzeB zu demonstrieren. Ebenso wird bel dieser Gelegenhelt die Verfremduns selbst 
- entsprechend der doppelten Struktur der Illusion - getelit. Zum AbschluB dieses Telles wird genauer auf den Inneren Zusammenhang von Illusion und Verfremdung eingegangen bzw. das Innere Verhaltnis zwisohen belden erlatert. 3. Tell, SchlieBlich wird die ron Brecht selbst angebotene Konkretion des Houptmittels der Verfremdung (vom geschriebenen Text aus gesehen) betrachtet (und dies voljzieht sich vorwiegend am Guten Menschen von Sezuan, 1ndem Brechts Methodik in der Sprachgestaltung im Hinblick auf die Verfremdung systematisoh untersucht wird.

In dieser Arbelt ware es noglich gewesen, stattdessen, daB $1 \mathrm{ch}$ von der Illusion ausgehe, von der viel erbrterten "E1nfthlung" auszugehen und damit den schon oft beschrittenen Weg zu gehen. Diese Rlohtung habe 1ch aber nicht elngeschlagen, um auch die selte der Illusion als solche, von 1hrer Beglelterscheinung (der Einfthlung) getrennt, zur Geltung kommen zu lassen. Dies hat zur Folge, daB $1 \mathrm{ch}$ mich nlcht in groBe Auselnandersetzungen mit den verschiedensten Interpretationen unterschiedicher theatra11soher Wirkungsintentionen hinsichtlich der Elnfuhlung (siehe u.a. Aristoteles, Cornelile, Lessins, Schadewaldt, Kommerell) einlesse. Die W1chtigkelt dieser Ausfthrungen mochte 1ch nicht abstreiten; meine Absicht hat eine positivere Motivation, namlich diese, daB $1 \mathrm{ch}$ daran glaubte, man könne auch im Anhalt an den Illusionsbegriff die Brechtsche Verfremdung behandeln, und zwar dahingehend, daB 
Brechts W1rkungsintention als Gegenbewegung auch gegen die Illusion (und nicht nur gegen die Elnruhlung) herausarbe1tet verden konnte. Dooh hoffe $1 \mathrm{ch}$, daB $1 \mathrm{ch}$ mich selbst nlaht in elne "Illusion" begebe, wenn loh melne, Ioh ware auf diese Welse zu fruchtbaren Ergebnissen ftr das Veratandnis des Brechtschen Theaters gekommen. 
INHALTSVERZEI CHNIS

Selte

vonwORT $\quad \ldots \ldots \ldots \ldots \ldots \ldots \ldots \ldots \ldots \ldots \ldots \ldots \ldots \ldots \ldots$

\section{ILLUSION}

EINLEITUNG

I. NICOLAI HARTMANNS THEORIE DES ASTHETISCHEN GEGENSTANDES

Zwelerle1 Gegenstand des asthet1schen Gogenstandes

Zwelerlo1 Seln des asthet1sohen Gegenstandes

N1colal Hartmanna Ablehnung der Illugion

II. KATE HAMBURGERS KONZEPTION DER ILLUSION..

III. ABSCHLIESSENDE BETRACHTUNG UND BESTIMRUNG DES ILLUSIONSBEGRIFFS PUR DEN WEITEREN VERLAUF DIESER UNTERSUCHUNG

IV. EINFUHAUNG IN DEN ILLUSTONSBEGRIFF

BEECHTS

V. UATERSUCHUNG DER ILLUSION BEZOGEN AUF B. BRE CHT

D1. theaterkonstitulerende Illusion und 1hre stelgerung .................

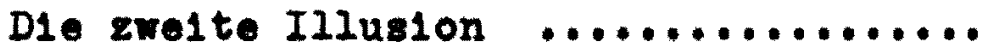

B. Breohts Intention hinsiohtlich der Illusion $\ldots \ldots \ldots \ldots \ldots \ldots \ldots \ldots \ldots \ldots \ldots \ldots$ 
VERFREMDUNG

EINLEITUNG $\ldots \ldots \ldots \ldots \ldots \ldots \ldots \ldots \ldots \ldots \ldots \ldots \ldots \ldots \ldots$

I. HAUFIGKEIT UND TRADITION DER KUNSTLERISCHEN VERFREMDUNG UND BRECHTS ABGRENZUNG DAVON ....

II. VERFAEMDUNG BEI BAECHT IM HINBLICK AUF

THEATRALISCHE HITTEL $\ldots \ldots \ldots \ldots \ldots \ldots \ldots \ldots \ldots$

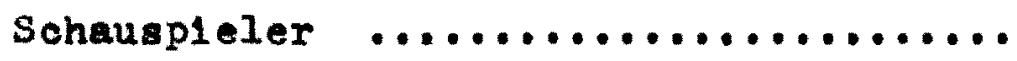

Buhnenbildner $\ldots \ldots \ldots \ldots \ldots \ldots \ldots \ldots$

Musiker und Choreograph,$\ldots \ldots \ldots \ldots \ldots$

III. VERFREMDUNGSPROZESS $\ldots \ldots \ldots \ldots \ldots \ldots \ldots$

Verfremdung auf der Ebene des Roflexes

(Vorgtufe) ...........................

Verfremdung auf der Ebene der Rerlexion.

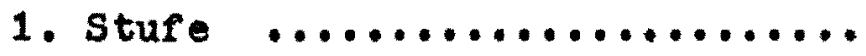

62

2. Stufe $\ldots \ldots \ldots \ldots \ldots \ldots \ldots \ldots . . . . .64$

3. Stufe $\ldots \ldots \ldots \ldots \ldots \ldots \ldots \ldots .6 . \ldots 6$

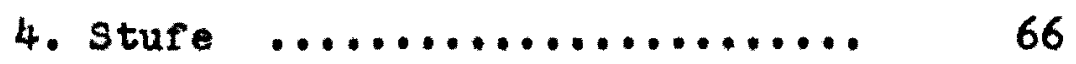

5. Stufe $\ldots \ldots \ldots \ldots \ldots \ldots \ldots \ldots . .6$....... 67

UNTERSUCHUNG DES HAUPTMITTELS DER VERFREMDUNG

EINLEITUNG $\quad \ldots \ldots \ldots \ldots \ldots \ldots \ldots \ldots \ldots \ldots \ldots \ldots$

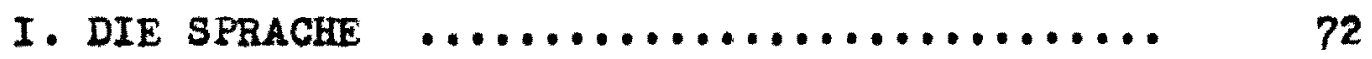

Festigke1t der sprachlichen Formulierung 74

Verfremdung selbstverstandilch gewordener Pormulierungen ..................

D1rekte verknderungen $\ldots \ldots \ldots \ldots . \quad 81$

Unangemessene Verwendungen ...... 85

Relat1vierungen durch Nachsatz ... 90

Wortliche Auffassungen $\ldots \ldots \ldots \ldots .92$ 
Verfremdung duroh neue verfremdende

Formulierungen $\ldots \ldots \ldots \ldots \ldots \ldots \ldots \ldots \ldots$

Scheinbar unangemesene Verbindungen zweler Begriff sfelder ......

Verbindungen zweler a1rekt gegensatzlicher Begriffe ..........

Verfremdende Verbindungen zreier sich direkt unterstutzender Beerifre

D1alekt1 ache Darstellungen eines elnzigen Begrifrs 
AN ABSTRACT OF THE THESIS OF Robert $E$. Folk for the Master of Arts in German presented May 10, 1972.

Title, Illusion und Verfremdung: Begriff. Form und Wirkang. Bezogen auf Bertolt Breoht, "Der gute Mensoh von Sezuan".

APPROVED BY MEMBERS OF THE THESIS COMMITTEE!

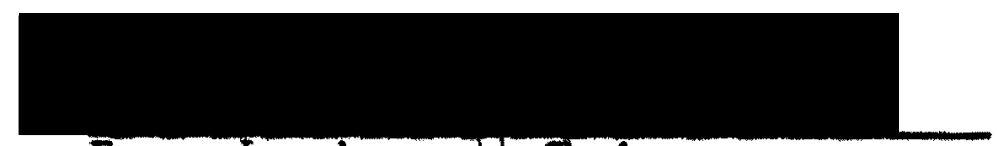

Franz Langhamery Chalrman

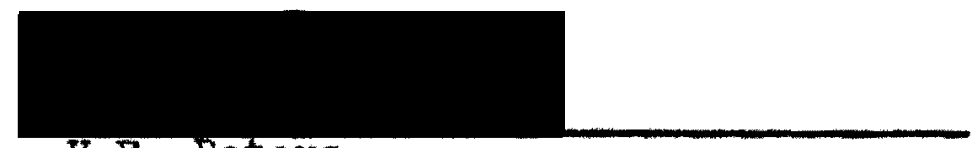

$$
\text { H.P. Poters }
$$

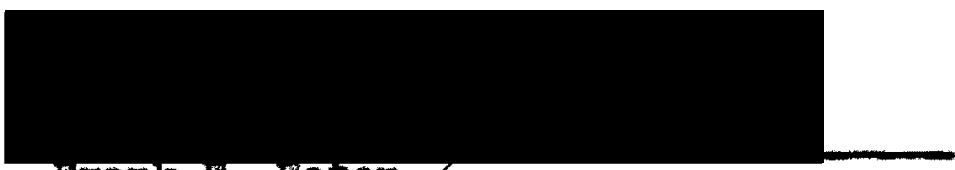

Perhaps Bertolt Brecht's most obrlous intention in connection with the theater was to help change the economio oonditions, under whioh the people in capltalistio soc1ety 11ve. But, according to h1m, this oould only be possible if the people were to develop a crition attitude and avareness of the contradictions that exist in such a soc1ety. In which "exploiters explo1t the explo1ted", to put it in Brecht'g atraightforward terminology. It was h1s goal in the theater to help the spectator achleve this orltical att1tude. Howerer, 1t was his opinion that this could only 
be done by rejecting the traditional theater of 1llusion, In which the spectator 1dentifies completely with the characters and 1s "manipulated" by the supposed foelings of the hero and by the supposed necessity in the order of occurrences and by the supposed necessity of the occurrences themselves. To be oritical means, of course, that not only are the feelings of the spectator involved, but also his thoughts. In order to introduce thought into the spectator, 1t was necessary for Breoht to break the spectator's 1llusion that he was partiolpating in the actions on stage and that these actions were real. Th1s led to the most significant of Brecht's theater contributions, which in English 18, perhaps, best called "allenation". Actually, 1t $1 \mathrm{~s}$ more acourate to say that h1s particular use of this element was h1s contribution. That is to say that his application of certain 1llusion-destroying teohniques was not simply for aesthetic purposes, but rather for socletal purposes. Each of Brecht's "alionations" leads in fact, in the 1deal theater situation, to revealing of some contradiction in the depicted societal conditions.

It was my goal in this thesis to analyze the "allenation" a countermovement to the theatrical 1llusion. In order to do this, it was necessary to ansiyze the theatrical 1llusion as well, which oomprises the first part of the thesis. The second pert is concentrated on "alienation". On a critical basis of acceptance and rejection of two se- 
lected thinkers on the subject of 111 usion I was able to arrive at a concept of 1llusion. whlch would also encompass Breoht's own writinge ooncerning the tople. Since Breoht did not research the concept of 11lusion in a systematic manner, it was disoovered in the course of researohing his writings that Brecht was referring to two different phenomena with the same word, "1llusion". Purther, I discovered that 1t was possible to divide his "alienation" technlque Into two different types, which corresponded to the ilmitation and hindranoe respectively of the two types of 1llusion.

I also attempted to oonstruct a step-by-step process, through whioh the sectator goes whenerer an "allenation" appears in a drama by Brecht. Th1s "allenation-process" begins with the application of the "alienation" and proceeds to the hoped for change in aoclety.

In the third and last pert of the thesis I analyzed the main medium of "allenation", which is the langrage 1tself. This was done with the purpose in mind of research1ng Brechts "allenation" method where it would be most fruitful. And since I was limited to the written text (a live presentation would reveal less of Brecht than of the director of the play), it was obrlously the most appropriate research object for this purpose.

In order to simpilfy it for the reader. I I1mited my referenoes to specifio dramas as muoh as possible to one play, namely "The Good Woman or Sezuan". 


\section{ILLUSION}

\section{EINLEITUNG}

Um in den Illusionsbegriff elnzurthren, mobhte $1 \mathrm{ch}$ zunuchst von spezifisohen Auffasengen dieses Begriffs ausgehen in der Welse, daB diesbeztglich eine kritische Auselnandersetzung ron Verstehenswelsen vorgenommen wird mit der $A b-$ sloht, das Verstandnis Bertolt Brechts von Illusion dadurch zu ermoglichen, daB zundohst durch Anlehnung an die dargestellten Verstehenswelsen oder Abhebung von diesen ein Begriff von Illugion erarbeltet werden soll. Davon auggehend bezlohe $1 \mathrm{ch}$ mioh zuerst auf N100lal Hartmann, dessen Ausarbeltung des Illusionsbegriffs meines Erachtens sohr gut dazu belzutragen vermag, dem Begriff (nach der selte der thusohung, um vorzugreifon) im allgemelnen zu verstehon, sodann ziehe $1 \mathrm{ch}$ kate Hamburgers Austuhrungen vor allem deshalb heran, well 1hre Auffassung sloh schon spez1f1scher der erarbelteten und der Brechtsohen Aurfassung annuhert.

I. NICOLAI HARTMANNS THEORIE DES ASTHETISCHEN GEGENSTANDES

N1colal Hartmann setzt in selner "Asthet1k" Illualon und Tluschung gleloh und halt sie far eln wesentliohes Moment des "Hegelschen Scheins", wobel das Dargestellte nloht da se1, aber dem Anschauenden so entgegentrete, als ware 
es real da. Dieses Phlnomen lehnt $N$, Hartmann fur die "oohte Kunst" ab aufgrund einer sehr kompliz1erten Kunsttheorlo, dle loh nur ganz kurz zusammenfassen werdel diese $\mathrm{Zu}-$ sammen fassung beschrlint sich auf das, was wir unbedingt brauchen, um seine Auffaseung von Illusion $\mathrm{zu}$ verstehen und sodann seine Ablehnung der Illusion begreiflioh zu machen.

Zwelerle1 Gegenstand des athet sohon Gegenstandes

Der Asthet1sche Gegenstand ${ }^{1}$ impliziert "zwelerle1 Gegenstand", elnen "realen" und elnen "1rrealen". Der reale Gegenstand 1st der, der alles elnsohlleBt, was der Botrachter wahmimmt, was den Sinnen gegeben 1at. Der 1rreale Gegenstand 1st der, der alles das umraBt, was rermittelst des Bealon ersohe1nt. Der 1rreale Gegenstand ist das Erscheinende, das in dem Berrubtsein des Betrachters hervorgerufen wird, in anderen Worteni der geistige Gehalt dea dsthetischen Gegenstandes.

Dieser Auftellung ontsprechend hat die Betrachtung eInes Aathetischen Gegenstandes "zwelerle1 Sohau", "Schauen erster Ordnung" und "Schauen zwe1ter Ordnung". Sohauen er-

1 Ioh verwende diesen Hartmannsohen Ausdruck hier m1t Bedenken, well der Ausdruck die sehr berecht1gte Frage fordert, "Was helBt athetisoh?" Ich hitte ach das Wort "schon" verwenden konnen, aber die Frage bliebe dieselbe. Es lohnt sich elgentiloh nioht fur unsere Zweoke das "Asthet1sohe" oder "das Sohone" sehr t1ef zu untersuchen, denn darum geht das ganze Buoh. Es gentigt far unsere Absiohten in bezug auf Illusion, diese Ausdrtcke mehr oder wenlger in der Form elner Vorausetzung vorzubringen. Derjenlge, der den Begriff in seiner Vollstundigkelt verstehen w11l, muB die Hartmannache "Asthet1k" selbst lesen. 
ster Ordnung 1st die sinnliohe Schau, d1e Wahrnehmung. Schauen zwe1ter Ordnung 1st das "spontan-1nnere Ersohauen" des Erscheinenden, die dbersinnliche Schau.

D1e Betrachtuns dieses obersinnlichen (des Irrealen) 1st ein produktives Soheuen, des nur in der Vorstellung fur das schauende Bemutsein besteht, "-- konkret und bunt, wie nur je das Erlebte .-., aber dennooh nloht erlobt, sondern spontan hervorgebracht...eln Gaukelwerk der Phantesie..."2 Kant nannte dies "E1nb1ldungskraft". Die Phantas1e ist kolne frele Phantasie, da sle durch das Sinnliche nach Hartmann "das Beale" bedingt 1st.

D1e Sohau zwe1ter Ordnung hebt das Schauen erster Ordnung "tber die alltagliche Hahmehmung hinaus und glbt [1hm7 den besonderen athetisohen Charakter;" os rugt "zum unmittelbar sinnlich Gegebenen etwas Neues" hinzu. 3 Dlese "zwelerle1 Sohau" bestimmt dann die Struktur des asthetisohen Gegenstandes, die Hartmann "zwelerle1 Gegenstand" nennt. D1ese Struktur, obwohl sle elne gespeltene 1st, wird als Einhe1t erlebt und emprunden. In der Natur z.B. wirken d1e Frahlingslandschart (das S1nnfallige) und die Frahlingsst1mmung (das Erscheinende) "als Einhelt, ohne Ldoke und Fuge". Im Gemblde etwa werden die Parbrleoken und die Leinwand mit der gemalten Landschart (dem Ersoheinenden) als

2 N1colal Hartmann, Aesthetik (Berlin. Walter De Gruyter \& Co., 1953), s. 18 .

3 Ebenda, s. 19. 
Einhe1t emprunden.

"D1ese E1nhe1t liegt in Erscheinen", sagt $N$. Hartmann. "Was erschelnen IABt, muB real seln, und was erscheint, kann nloht real soin, denn es besteht nur in diesem selnem Ersoheinen." 4 sie alnd untrennbar mitelnander verbunden.

\section{Zreierle1 Soin dee sothetischen Gegenstandes}

Dooh, obwohl der Asthet1eohe Gegenstand zwelerlel Gegenstand 1mpliz1ert und darstellt, dessen zwel Bestandtelle als Elnhelt wirken, hat der Gegenstand "zwelerlel Seln", das sloh gespalten manifestlert, und zwar als eln "reales" und ein "1rreales Se1n". W1r sind uns dieses "Gespaltenseins" Imer bewuBt. Nach der Selte des zwelerlel Gegenstandes des Kunstwerkes sind die gemalte Landschaft und das Material, das dazu benbtigt wird, sie darzustelien, nicht trennbar vonelnander, sondern bedingen sich vielmehr notwendig gegenseltigi denn die gemalte Landschart ist 1hrerselts aur das Material angewlesen, whrend andererselts das Material von der gomalten Landschart abhangig 1st insorern, als diese $1 \mathrm{hm}$ erst seine Form verleiht, belde zusamen reprasontieren die Elnhelt des asthetisohen Gegenstandes. Jedoch nach der selte des zweierle1 Selns des athetisohen Gegenstandes wissen wir sehr wohl, daB die erschelnende Landsohart (gemalte Landschaft) nicht die reale Landschaft in unserem Leben 1st. Im Drama zum Belapiel wirken elnerselts

4 Hartmann, s. 34. 
der erschelnende Wallenstein und der Schauspleler (das Rea1e) als Einhelt, der Eine erschelnt in Anderon. Dooh andererseits 1st uns standig gegenwirtig, daB der duroh den Schauspleler erschelnende Wallenstein nloht der Wallenstein als soloher 1st oder, um es kantisoh auszudrtioken, nioht der Wallenstein "an sich" 1 st.

N. Hartmann nennt dieses Gespaltenseln "ein schrobendes Dase1n". Der Gegenstand 1st da und auch nicht da. D1eses Schweben emprinden wir im Sohauen und im GenieBen als "Zauber des Sohbnon". "Nur Indem wir [den Gegenstand7 als ungestorte Elnhelt erloben und dennoch den Gegensatz von Dasein und Niohtdasein in ihm spdron, konnen wir die Magie des Erschelnungsverhultn1sses erfahren." 5

Vom berelts Gesagten ausgehend, kann jetzt die Illusion etwas deutlicher gemacht rerdeni Die Illusion (TAuschung) 1st nach Hartmann vorwlegend dadurch bestimmt, daB das gedoppelte Dase1n (das sohwebende Dasein) des asthet1schen Gegenstandes fúr den Betraohter aufgehoben 1 st.

\section{Nicolal Hartmanns Ablehnuns der Illusion}

D1e Hartmannsche Ablehnung der Illusion kann in vier Granden zusammengeraBt werden.

1) Die erste Begrandung beruht in Wesentlichen daraur, daB die Einscheltung der Illusion die Ausschaltung der Asthet1k bedeutet. Wenn die Emprindung des gespaltenen Da- 
selns bel der Betrachtung elnes Kunstwerkes fehlt, nimnt das Erscheinende einen W1rkliohkeltscharakter an, oder in anderen Worten: Das Erscheinende verliert seinen Nichtdeseinsoharakter, so daB nur noch der Daseinsoharakter tbrig blelbt. D1e Illusion, so hat slch orgeben, verhindert gerade das Asthetische Schauen und macht das Kunstwerk zum N1cht-Kunstwerk.

2) Naoh $N$. Hartmann wire die Illusion in allen Ktinsten ohnehin unmoglich. Indem der Betrachter sich lmmer dieses Gespaltenselns zwischen dem realen seln und dem 1rrealen Seln bemut 1at. "Kelnem Betrachter wird es einfallen, das Landschaftsbild fur die reale Landschaft, das Portrat fur die Person zu halten." 6 Und um dies auf das Theater zu bezlohen, Kelnem Zuschauer wird es elnfallen, die durch den Schauspleler ersohelnende Flgur fur die reale Flgur zu halten, "auch wenn es noch wahr 1st, daB der hingegebene Betrachter seine reale Umwelt verg1Bt", denn oln Rest dos UmweltsbewuBtseins bleibt immer vorhanden.

3) Sogar wenn es ktinstlerlsch mogllch wire, diesen Best des Umweltsbewabtselns zuniohte zu machen, ware es moral1soh unmbglioh fur den Betrachter, dlese vorgetusohte W1rklichke1t zu genieBen, d.h. rtr den Betrachter "gelassen dazust.tzen, genleBend sohauend und horend, Zeuge rafrinierter Intrige oder gar des Mordes und Totschlages zu se1n*.?

6 Hartmann, s. 36

7 Ebenda, s. 111. 
Es ware unmoglich selbst im Fall "nur tiefen seelischen Le1dens. Die Bthne warde damit auch eine ganz ralsche Zumutung an Ihn stellen. Der Sinn des tragisohen Spleles muBte sioh in sittliche Rohelt verkehron, der des komisohen in Herzlo81gke1t. Keln Theater mutet dem Zuschauer so etwas zu." 8 Vlelmehr gibt es "In der Schausplelkunst Elnsohrankungen des Reallsmus, die St1lislerung der Sprache duroh den Vers, dos Buhnenb1ldes durch die gerahmte Blldhartigkelt selbst, durch dle Rampe, und manches mehr" 9 wodurch schon der Zusohauer daran gehlndert wird, sloh in die auf der Buhne dargestellte S1tuation vollkommen hinelnzuleben.

4) M1t diesem Punkt lst der v1erte Grund N. Hartmanns Ablehnung der Illuaion eng verknipft. Denn ein Grund, warum w1r in das Theater gehen oder aberhaupt die Kunst betrachten, besteht doch darin, ein wenlg unsere Umwelt zu vergessen, "eln Abrallen des Alltags und der Sorge von uns, ein Gejostsein und eine Entspannung" 10 zu finden. "W1r flachten uns in diesen schwebenden Zustand, wenn wir dem Drang und dor seelisohen Belastung entrliehen wollen,"11 Nach N. Hartmann "sohlelcht sich der Irrtum erat ein, wenn wir das als Flucht in elne Welt des Scheines deuten wolien.

8 Hartmann, S. 111. Hartmann hat hier d1e moral1sohen Aspekte nicht veiter herausgearbeltet.

9 Ebenda, s. 36.

10 Ebenda

11 Ebenda 
Handelte sich hier wirklich um Schein oder Illusion, so whrden wir nur elne Last mit einer anderen vertauschen, wir whion das Erschelnende fur real halten und damit ein neues Elngeapanntseln erfahren."12 Damit will Hartmann sagen. ware es schon, moralisoh mbglioh und tberhaupt moglich in der Kunst Illusion zu haben, gelinge es uns nicht, dem E1ngeapantsein der allagliohen Belastung zu entfliehen (elne 21elrorstellung, die anschelnend noch innerhalb des Hartmannschen Begriff der asthetischen Betrachtungswelse orlaubt 1st).

Was nach melnem Ermessen an dieser Aufrassung der IIlusion zu kritisieren 1st, 1st das Folgende. Es wird deut$11 \mathrm{ch}$ be1 $\mathrm{N}$. Hartmann m1t der ersten Erwannung dieses Wortes "Illusion", daB er damit elne totale Illusion, elne rollkommene Hingabe des Betrachters an die Tuschung aber die Wirklichke1t, ohne Rulokkehr in die Bealitut meint. Mit einer solohen Auffassung des Begriffs gibt es naturlich kelne Illusion in der Kunat. Aber es 1st gerade dieser "SchwarzWe1B-Gebrauch" des Begriffs, der slch fur unsere Zwecke aussohl1eBt. D1ese Konzeption laBt keinen Raum fur einen Greduntersohied der Illusion Ubrig, den Hartmann selbst 1mplizlert aber nloht zugibt. Das "Vergessen der Umwelt" stellt fur Hartmann keine Illusion dar, solange der Betrachter ein ganz winziges Kernohen des RealitutsbewuBtselns hat, Aber

12 Hartmann, s. 36. 
er verfangt sich in elner widersprtichlichen Ausdruokawelse, wenn or auf der elnen Selte dio Illusion verleugnet, aber s1. dann spler mit den Worten "Vergessen der Umwelt" herelniubt. Denn, wenn der Betraonter anluBlich des Kunstwerkes seine Umwelt vergiBt, mag os auch nur tellwelse sein, dann hat dies solnen Grund darin, daB die Illusion zum Te1l w1rksam geworden 1st. Es w1rd spter In dieser Untersuohung erluutert werden, daB selbst Brecht Cen Begriff kelneswegs so streng auffaBt, wenn or durch den Dramaturgen in Messingkauf sagt, "...es handelt sloh um elnen Graduntersohled der Illusion." 13

II. KATE HAMBURGERS KONZEPTION DER ILLUSION

Kulte Hemburger unterscheldet in ihrer Logik der D1chtung, anders als N, Hertmann, zwlochen Taschung und IIlusion in wesentlicher Hinsicht, diese Untersoheldung beruht aur der ron $1 \mathrm{hr}$ gesetzten Trennung zwisohen elner "Als ObStruktur" und einer "Als-Struktur".

D1. Als Ob-Struktur betrifft die Thuschung insorern, als diese etwas darstellt, als ob os die Wirklichke1t ware. D1es besagt, daB die Als Ob-W1rkl1ohke1t nicht die W1rklichke1t 1st, die sie vorgibt zu sein. $K$. Hamburger nennt in diesem Zusammenhang des Belsplel von den K1rsohen des Malers Zeuxis, die die Spatzen getulusoht hatton, woll die Spatzen

13 El1sabeth Hauptmann. Bertolt Breaht: Gesammelte Werke (Franirurt, Suhrkamp veriag, 1967), XVI, 515. 
81. Itr echte Kirschen gehalten hutten. D1e Als-Struktur hingegen, die man etwa belm Drama findet, erzeugt keine Tuuschuns, wohl aber elne Illusion. Whicend die Als Ob-Struktur In Form des Konjunktiv 1 rrealis beschrieben werden muB, we1l sie das Bedeutungamomont der Thusohung onthalt, drtokt d1e Als-Struktur die tatslohliche W1rkl1chke1t aus, nimlioh d1e N1oht-W1rk11chke1t der Daratellung. "D10 Als-Wirkilohke1t aber 1st Schein, Illusion ron Wirkllohke1t, und das he1Bt N1oht-W1rkliohkelt oder Fiktion."14 Sohillers Maria Stuart erscheint nicht, als ob sie die wirkliche ware, sondern als Theaterfigur, die den "Schein ron W1rkliohke1t" erzeugt. "Auch das Murchen ersoheint als Wirklichke1t, solange wir lesend oder zusohauend in inm rerwellen, doch nloht so, als ob os elne wirklichkelt ware." 15

Um zu dem Verstundinis dieses Zusamenhanges der belden W1rkl1chke1tsstrukturen zu gelangen, muB darauf reflekt1 ert werden, daB es hier um zwel rersohledene W1rklichkeltsberelche geht, die nur in einer ungekehrten Proportionalitat zuelnander stehen. Die elne W1rkilchkelt bezioht sloh auf d1. W1rkl1chke1t, die nachgeahmt wird oder werden soll, die andere auf die W1rkllohkelt des Theaters, d.h. auf die W1rk11chkelt, daB sioh der Zusohauer in einem theater befindet. Dadurch, daB die W1rklichkelt, die nachgeahmt wird oder wor-

14 kute Hamburger, D1e Log1k der D1chtung (stuttgart, Ernst Klett Verlog, 1968), S, 55.

15 Ebenda 
den coll, als Wirklichkelt negiert wird, und das bedeutet, daB der Zusohauer s1ch dessen gewiB 1st, daB die dargestellte wirklichkelt nur eine nachgeahmte und also oine scheinW1rkl1chke1t 1st, die als N1cht-W1rklichke1t mit der Wirklichke1t, daB der Zuschauer im Theater sitzt, zusamenfallt, kommt es zu elner gedoppelten BeruBtwerdung des zuschauers 1m Hinbliok auf die Wirkliohke1t.

III. ABSCHLIESSENDE BETRACHTUNG UND BESTIMMUNG DES ILLUSIONSBEGRIFFS FUR DEN WEITEREN VERLAUF DIESER UNTERSUCHUNG

Aus dleser Gegentberstellung der belden Theorien wird ersichtlich, daB K. Hamburger und $N$. Hartmann unter "Tluschung" dasselbe rerstehen, nlmich elne Tauschung im tblichen Sinne elnes Trugsohlusses. Whrend aber Hartmann die Illusion und de Tuschung glelohsetzt, unterscheldet $k$. Hamburger diesbeztglich wesentlich, und zwar in der Welse, daB fur sie der Begrife "Illusion", um Ihre Konzeption zusamenzufassen, das Bematseln der Thuschung oder das BemuBtse1n der N1cht-W1rkl1chke1t bedeutet.

W10 1ch schon erbrtert habe, kann $10 \mathrm{~h} \mathrm{~N}$. Hartmanns Auffasaung ron Illusion gerade wegen der darin liegenden terminologisohen E1nseltigkeit nicht zustimmon, mobte jedoch herausstellon, daB das, was or mit "Vergesson der Umwelt" melnt, elne groBe Rolle rar melne Aurfasung spielen 8011. Was K. Hamburger anbelangt, so mobhto $1 \mathrm{ch}$ betonen, daB $1 \mathrm{ch}$ ihre Verstehenswelse beztglich der Illusion tbernehme mit dem grundlegenden Zusatz, daB loh nioht nur das Be- 
muBtsein der Teusohung in dem Begriff "Illusion" enthalten sehe, sondern auch das Moment der Tuschung selbst. Wenn $X$. Hamburger von "verwellen" sprioht, so finde loh berelts da das Moment der Tuuschung als ein in die Illusion integriertes Moment angelegt, da in dem Wort "verwellen" auch dies, daB zum Tell die Umwelt vergessen w1rd, liegt, woraus rolgt, daB zum Tell elne Tauschung zustandekomet. Aber diese Selte wird von $K$. Hamburger nloht welterentwiokelt. Ioh hingegen will dieses Moment als Bestandtell des Illusionsbegriffs selbst zur Geltung bringen. Ioh sehe die Illusion als einen Begriff, der zwel scheinbar widerspritohliche Komponenten hat, nlmlich die Tluschung als TrugschluB und das BeruBtseln der Tuschung. Dleses Bewrutseln hebt wiederum zum Tell die Tauschung auf, aber nloht in vollkommenem MaBe, denn das Bemutsein der Tasohung Implizlert doch die Anresenhe1t der Tauschung und laBt dieses merkwirdige Verhaltnis nicht versohwinden.

Welterhin 18t es wichtig, die umgekehrte Proportionalitat dieses Verhaltnisses herrorzuheben, und zwar bedeutet eine Stelgerung in der Tluschung zugleloh olne Verminderung des Bemutseins lber die Tuschung. Dles gilt ebenso rur die entgegengesetzte Selte insofern, als elnem starken BewaBtsein ther die Tauschung oine schwache Taschung entspricht. Man kann also zur Verdeutlichung dieses verhaltnisses sagen: Je mehr der Zuschauer glaubt, die dargestellte Marla Stuart sel die reale historisohe F1gur, desto we- 
n1ger 1st er sich dessen bemaBt, daB sle elne von der Sohauspielerin gespielte Figur 1st. Es 1st abrigens ein Zlel dieser Arbelt, eventuell zu zelgen, daB es in bezug auf dio Illusion elgentlich nur die E1nseltigkelt dieser Proportional1tat (1m Ausgang ron der Starke der Tusschung) 1st, die Breoht stort, nlcht, daB es tberhaupt Illusion glbt, und dab es elne voll1g andere Art von Illusion 1st, die Breoht vollkommen ausachalten will.

Besonders mbohte $1 \mathrm{ch}$ darauf hinwelsen, um den Illus1onsbegriff ftr seinen Gebrauch auf einen Nemner zu bringen. daB, spreche $1 \mathrm{ch}$ ron elnem Zustandekommen oder E1nschrlinken der Illusion im Theater, von der Entstehung oder Einsohrlnkung elner Thuschung die Rede ist, mit dem Faktum im Hintergrund, daB diese Tluschung mie elne rollkommene 1st.

B1sher habe loh von Illusion geaproohen, als wre sle 1m Drama lediglioh eln moglichos Element. Jetzt moohte $1 \mathrm{oh}$ aber elnen schritt weiter gehen und das Fehlen ron Illusion ausachlieBen, denn melnes Erachtens kann es keln Theatersttlok ganz und gar ohne Illusion geben. Zwar fahrt die Absicht, die Illusion zu begrenzen, wie Hartmann mit Recht sagt, zu einer Elnsohrankung des Realismus in Drama ${ }^{16}$, aber solange nooh im Sttick eln gewisser Grad ron kealitatsahn11chke1t vorhanden 1st, gibt es elnen gewlssen Grad ron Illusion. Es 1st nun nloht moglioh, alle Realitatsthnliohkelt aus dem Stlck aususchalten, da das Theater gezmangen 
1st. Menschen als solche sprechen und agleren zu lassen. Sobald wir als Zuschauer Abblldungen von Menschenwesen sehen, machen w1I Assoziationen, die uns auf die Realitut verwelsen. DaB auch Brecht die Illusion daher nur begrenzen konnte, 1st von daher einleuchtend. DaB er sie nur begrenzen wollte, wird nooh gezelgt.

Whrend nach Hartmannscher Auffassung die Illusion entweder nullprozent1g oder hundertprozent1g 1st, das helBt, daB entweder "kelne Illusion" oder "totale Illusion" vorliegt, verstehe 1oh also unter Illusion alle Grade auBer null und hundert, in anderen Worten, man kann demzufolge von "wenlg Illusion" oder "starker Illusion" sprechen. Wenn loh von der Starke der Illusion rede, beziehe loh mich auf die Sturke der selte der Tuschung gegentber dem BemuBtseln aber d1e Tauschung.

Akzeptlert man die Mogliohke1t dieses Gebrauchs des Begriffs "Illusion", dann hatte man den Vorte1l, auch dann ron Illusion sprechen zu kblnnen, wenn es im Zuschauerraum nur eln ganz winziges Kernchen "Vergessen der Umwelt" glbt. D1es 1st elner Flasche analog, die nur ein Molekal Luft enthalt. D1e Antwort auf die Frage: "G1bt es Luft drin?" maBte, streng genommen, "Ja" sein, obwohl sle schwleris zu messen ware, und obrohl es dann, wenn die Existenz dieses elnzigen Molektls festgestellt ware, elnem viellelcht sohwer fallen warde, d1ese Prage mit dem elnzigen Wort "Ja" zuzugeben ohne erglinzenden Kommentar. 
B18 zu diesem Punkt haben w1r folgendes tber die IIlusion in Thester restgesetzt: 1) daB sle Gradunterschiede hat, jo nach der Theatersituation, 2) $A a B$ sle nlemals (1m Theater) elne hundertprozentige seln kann, 3) daB sie niemals aus elner Theatersituation rollkommen ausgesohlossen seln kann. Daraus erg1bt sloh, daB die Illusion ein sohrebender Zustand des Zuschauers 1st, in dem or tells s1ch se1ner realen Umwelt bemuBt 1st und sie tells verg1Bt, well er gelegentlich und zu elnem gewlssen Grad die Bthnengeschehnisse und Bahnengegenstande nicht far Reprasentanten elner nachgeahmten Reall tut hilt, sondern fir ursprtingliche und elnmalige Erelgnigse.

An dieser Stelle molchte ich einen kurzen Vorblick aur den welteren Verlauf dieser Untersuchung geben. Es soll Im Folgenden der Versuoh unternommen werden, den Illusionsbegriff, so wle er konstatiert worden 1st, auf B. Brechts Konzeption zu abertragen, das bedeutet, daB es meine Aufgabe seln wird, Brechts Auf fassung von Illusion in den erarbelteten Begriff zu 1ntegrieren. Zunilchst w1ll loh aber auf die psychologische Bes1s der Illusion elngehen, um sodann d1e konkrete Analyse der Illusion welterzurthren.

IV. EINFOHRUNG IN DEN ILLUSIONSBEGRIFF BRECHTS

Es ware Interessant, zu analysieren, was im Zuschaver selbst vorgeht, wenn die Illusionlerung zustendekomit. Aber es ware eine sehr komplizierte psychologische Unter- 
suchung, und Im Detall Ifge os auBerhalb des Berelohs der elgentlichen Absioht dieser Untersuchung. Es gentigt, glaube 1oh, nur elniges kurz zu erwahnon.

Je starker die E1nb1ldungskraft (d.h. die Phantasie) des Zuschauers 1st, desto starker wird foder wonlgstens kann) die Illusionlerung sein. Es ist hier bemerkenswert, daB man "passive" ron "aktiver" Phantasie unterscheldet. Passive Phantasie 1st nachrollziehend, d,h, eine Phantasle, die gewisse Informationen elner rixtiven situation geliefort bokommt, und die durch inre Iraft das Versetzen des Zuschauers in diese fiktive sltuation ermoglioht. Akt1ro Phantas1e 1st "d1e sohopferisohe Phantasio als olne der Voraussetzungen des Diohters, die nur duroh die Darstellungamogliohke1ten beschrinkt w1rd*.17 Diese 1st eine produkt1ve, kritioche, aussortierende Phantasie, Es 1st sehr wicht1g, diesen Untersehied zu erkennen, wenn man die Brechtsohe Theor1e 11est, denn manohes konnte sonst m1Brerstanden werden.

In "Das moderne Theater 1st das ep1sche Theatex", in dem Brecht erklart, warum das Theater sloh (als Eraatzbefrledigung) so sohleoht ron der Illusion 16st (nimlioh wo1l das Leben so schwer 1at und uns zuviel Sohmerzen bringt), spricht or aber "IIIusionen gegen die Realitat" in dor Kunst. d1. "nicht minder psyohisch wirkam" alnd, "dank der Bolle,

17 Gero ron W1lpert, Sechworterbuch der L1teratur (Stuttgart, Alfred kroner verlag, 1969), s, 566. 
die die Phantasie im Seelenleben behauptet hat". Mit diesem Satz verwelat er auf Freud (Das Unbehagen in der Kultur), der sagt, "Das Gebiet, aus dem diese Illusionen stammen, 1st das des Phantasielobena... Unter diesen Phantaslebefrledigungen steht der GenuB an Werken der Kunst, der... dem nlcht selbst Schopferischen duroh die Vermittlung des Ktinstlers zugangl1ch gemacht w1rd."18 H1er 1st offenbar d1e "passive" Phantasie gemelnt, und gerade diese Form wollte Brecht durch eine verbesserte Gesellsohart ganz unwlohtig ftur das Theater machen.

So sagt or 1m Yessingkauf, "Es 1st ein groBer Untersohied, ob jomand eine Vorstellung ron otras hat, wozu er Fhantasie braucht, oder eine IIIusion, wozu er Unverstand brauoht. Wir brauchen fur unsore Zwecke Phantasie, auch den Zuschauer wollen wir elne Vorstellung (Hervorhebung ron mir) ron elner Begebenhelt rermitteln, nioht elne IlIusion erzeugen." 19

Es 1st klar, daB, obwohl der erste Tell dieses zitats In Kontext des Megaingkaufs ich auf den Sohauspleler bez1oht, er auch auf den zusohauer abertragbar 1at, und daB d1ese Phantade die "akt1ve", die sohopferische 1st. D1ese akt1ve E1nb1ldungskrart entspricht dem berahmen "dr1tten Vers", den der Leser oder Zuschauer selbat in seiner

18 Anna Freud. S1gmund Freud, Gesammelte Werke (London, Imago Publishing Co., Itd., 1955), XIV, 439.

19 Hauptmann, XVI, 583. 
Vorstellung dichten soll. Marlanne Xesting bringt in Das eplache Theater mit dem Brechtschen Gedioht "Ulm 1592" ein glenzendes Belspiel des "dritten Verses":

"B1 sohor, $1 \mathrm{ch}$ kann fllegen", Sagte der Sohnelder zum Blsohor. "PaB auf, wie $1 \mathrm{ch}$ 's machl" Und or etieg mit so nen Dingen, Die ausahn wie Schwingen, Aur das groBe, groBe Kirohendach. Der Blsohof ging relter. "Das sind lauter so Lagen, Der Kensoh 1st kein Vogel, Es wird nie eln Mensoh fliegen". Sagte der Blschof zum Sohnelder.

"Der Sohneider 1at versohleden", Sagten die Loute dem B1sohor. "Es war elne Hatz. Selne Flugel alnd zerspellet. Und er liegt zersohellet Auf dem harten, harten K1rohenplatz," -D10 Glocken solien luten, Es waren nichts als Ligen, Der Mensoh 1st kein Vogel. E. wird nie oln Monsoh rliegen"

"D1e dritte Strophe, die besagt, daB heute die Monschen fliogen und der Blsohof unreoht hatte, 1st ron Breoht ausgespart, der Leser hat sie selbst zu rollz1ohon."21 D1eses Art1vwerden des Zuschauers, man konnte rast "M1tdichten" sagon, war ein zlel Breohts und 1st ein wesentliohes Merkmel in seinen melston Worken und wird auch in anderen Formen auBer dem "dritten Vers" versuoht.

20 Marlanne Kesting, Das epleche Theater (stuttgart, W. Kohlhammer Verlag, 1969), $3.62 \mathrm{f}$.

21 Ebenda, s. 63. 
Wenn B. Brecht rom "Urte1l", wle in "Klelnen Organon"Z2 oder vom "Kritiziamus" in Messingkaur 23 sprioht, meint or dis schaffende Phantasie, die sohopferlsche Vorstellung von elner anderen Daratellunganoglichke1t. Diese schopferische Vorstellung bewirkt wenlg Illusion, ja, verhindert sle sogar fur den Vorstellenden. Die andere, nachrollziehende Phantasie 1st es, die zum starisen Vergessen der Umwelt fuhrt. Sle tut das duroh elne "Elnfthlung", d.h. ein gerthlomaBiges, unreflektiertes Ergreifen der Personen und Situationen des Drames, in anderen Worten, durch ein Hineinverlegen des Ich in das Drama. Diese passive Phantasle lat es, die die starke Illusionierung ermoglicht.

Nachdem ich die Illusionterung als ProzeB des Zustandekommens der Illusion kurz bohandelt habe, mbchte loh nun auf die Illusion, die sioh so gesehen als Endprodukt der Illusionlerung gestaltet, zurackommen, um diese welter zu untersuchen und auf Brecht anzurenden.

Im nachsten Abschnitt wird os un eine Analyse der I1Iusion auf inre struktur hin gehen mit der Absicht, die Aurfassung Brechts hinsiohtlioh der Illusion mitelnzubezichen, un letzlioh Brochts Ablehnung der Illusion (genauer, elnen bestimmten Tell der Illusion betreffend) gegentber deutlioh

\footnotetext{
22 Hauptmann, XVI, 694.

23 Ebenda, S. 651 .
} 
machen zu können.

V. UNTERSUCHUNG DER ILLUSION BEZOGEN AUP B. BRECET

D1e theaterkonst1tulerende Illusion und 1hre stelgerung

Insofern das Vorhandense1n, Sprechen und Agleren von Menechenfiguren auf der Buhne, zumindest von anthropomorphischen Gestalten, rar das Theater unentbehrlich sind. mochte $10 \mathrm{~h}$ sie als theaterkonstitulerende Momente kennzelehnen. De die Illusion elne autoratisohe Folge daraus ist, daB aufgrund dieses Faktums Assoziationen auf das reale Leben unvermeldilch sind, wodurch elne Einfuhlung in diese Mensohenfiguren zustandekommt und zuglelch der Eindruok, es handele sloh un die Boalitat, kobnen wir auch diese Illusion, man habe os mit der Realltat zu tun, als theaterkonst1tulerend bezelchnen.

D1e Illusion auBert sioh in der Glelohsetzung von

1) Schauspieler und Figur, 2) Vorfthrung und Geschehn1s,

3) Buhne und realem ort usw. Sie w1 rd aus dem oben angegebonen Grund hervorgerufen und kann in ihrer Intensitat gestelgert werden. Das bedeutet, sie hat Graduntersohiede, d1. durch die rolgenden N1ttel charakterlsiert werden. 1) durch die Wahrsohelnliohkelt (Realitatsahnlichke1t) des Inhalts und der Darbletungsform 24,2 ) duroh bestime

24 Unter Darbletungsform verstehe 1ch alle poet1schen und theatralischen Mittel, die verwendet werden, um den Inhalt zu bertragen. Obwohl belde Elemente (Inhalt und Darbletungsform) oft vonelnander untrennbar sind, z.B. In 
Darbletungsformen in der Gestaltung der Figuren (ror allem psychologische und theatralische), die spez1fisch darauf abgezielt sind, eine starkere Einfuhlung zu erreichen, und 3) durch bestimmte Darbietungsformen, die spezifisch dazu benutzt werden, elne Notwendigke1t des Verlaufs der Bthnengeschichte glaubhaft zu machen. Die Wahrachelnlichkelt (im Inhalt und in der Darbletungsform), obwohl sie in der Tendenz durch inre Realitutsahnlichkelt die Illusion ste1gert, well mehr Assoziationen auf das reale Leben dadurch ermoglicht werden, kann doch ron dem zwelten und dritten Element praktisoh unwichtig gemacht werden, namlich durch eine speziflsch abgezielte, besonders starke Elnfthlung und durch eine geschickte Zusammenverimipfung der Geschehnisse. Diese Konzeption wird verstandicher, wenn man slch elne Skala der Wahrscheinlichkelt rorstellt, ale sich ron Wahrschelnlichke1t an dem oinen Ende b1s zu Unwahrsohelnlichke1t am anderen hinzieht.

bezug auf die Sprache in einem Kunstwerk, konnen wir sie provisorlsoh um der Klarhelt willen scheiden. Inhalt ist der stoffliche, wertfrel nacherzahlbare Tatsachenablauf dea stuokes samt dem gelstigen Gehalt (Lebenshaltung, Weltanschauung usw.). Eine solche Trennung in der Sprache wire vereinfachend gesagt die folgende: Wes gesagt wird und was damit somelnt 1st, gohoren zum Inhait. H1e os gesagt wird lob Prosa oder Vers, ob gesprochen oder gesungen, ob geschrien oder geflastert) gohbrt zur Derbietungsform. D1e Tatseche elner Handlung und was die Handlung geistig repr:sentlert, entsprechen dom Inhalt. Wie diese Handlung dargestelit ird, 1st wiederum Darbietunssform. Der dramat1sohe Ort wire Inhalt, wahrend das theatrallsohe Aussehen dieses Ortes (Buhnenbild) zur Darbietungsform gehbrte usx. 
Da $1 \mathrm{ch}$ in dem, was Breoht uber diesen Zusammenhang sagt, elne Jbereinetimmung mit meiner hier vorgelegten skaIa sehe, unteratutze loh in Folgenden meine Konzeption mit einigen seiner elgenen Austahrungen.

Im Naturalismus, in dem eine extrene Wahrecheinlichke1t. ja elne photographische Genaulgke1t angeatrebt wird, wrde man festatelien kbnnen, daB alle dre1 11luaionaste1gernden Homente (Wahrscheinliohkelt des Inhelts und der Darbletungeform, atark abgezielte Elnfahlung und angertrebte Tauschung einer Notwendigke1t des Ablaufa) in atarken MaBe gegenwurtis alnd, mit besonderer Betonung auf dem ersten und aritten Punkt. D1e Wahrsoheinliohke1t wird duroh die Genaulgre1t des abbildes erreloht. Nichts wind dargestelit. was nloht in realen Leben vorkommen whrde. In Gegente1l oogar, d1e Darstellung soll elne direkte Wldersplegelung des Lebens seln. Diese Wahrsohelnliohkelt versturkt de EInfahluns, die owleso schon durch pajchologisch-ktnstlerische M1ttel angestrebt w1rd. Denn duroh die extrene Rea11tatsuhnl1 chke1t bekomint der Zuschauer d1e grobte Bas1: flir Assoziationen af das reale Leben, die auch ala Basis rur die Einfunlung dient. Das dritte Moment (die getauschte Notwendigke1t des Ablaufs) wird auf die folgende Welse erreloht, D1e "photograph1sohe" Teohn1k des Neturalianus erzeugt den moligen, autorituren Sohein einer exaiten W1edergabe der W1rkliohke1t, und ole ist nur ein Scheln, denn obwohl die dargesteliten Dinge. Verhiltnisee und Geschehnis- 
se erakt abgeblidet werden, 1st dies nur ein Te1l der W1rk110hke1t, nioht die ganze Wirkilohke1t, nimlioh olne ganz spez1f1sche W1rkl1ohke1t, die den Zreoken des Dramat1kers dient. B. Brocht sagt in Messingkauf: "Die (naturalist1sohen, RF) Sttokesohrelber arranglerten nattrich die Vorglnge obenso fle1Big wie die nichtnaturalistisohen. S1e rombinierton, 11eBen weg, veranstalteten Zusammentreffen von Personen an unwahrachelnlichen stellen, rergrbberten die e1non Vorgange, verfelnerten d1e anderen und so welter."25 Bestimmte unveranderliohe situationen werden dergestellt und durch d1. Sohein-Autoritat der photographisohen W1edergabe verfest1gt. "Das Publ1kum murde dezu gebrecht, elne ganze Wenge unhaltbarer Zustande, nun, zu rthlen, daB sie eben unhaltbar waren."26 Auch die starke Elnfahlung in d1. F1guren hat elnen rersohlelernden Effekt in bezug auf die Kausal1 tat der Geschehnlsse, we1l der Zuschauer m1t selnen Inw aufgezwungenen Gorahion wenig Mbglichkelt hat, dartber zu rerlektieren, ob der dargestellte Ablaur der Gesohiohte notwend1s sel oder nioht, Er 1st, Brechts Melnung nach, zu sehr in die gegenwitigen Probleme der Plguren gerthismiBis verwlokelt. B. Brocht sagt in keseingkauf, "In ouch (die Naturalisten, $R F$ ) fuhlte man alch $01 n$, und in die Welt riohtete man aloh $1 \mathrm{n}$. Ihr wart, wio ihr wart, und die Welt

\footnotetext{
25 Hauptiann, XVI, 515.

26 Ebenda, 3.517.
} 
bl10b, w1e s1e war."27

Aber gehen wir auf dieser skala elnen kielnen Sohritt in die Blohtung der Unwahrsoheinlichke1t, Im Realiamus nim11ch, der, st1ltypolog1sch gesehon, dem Naturallswus sehr nahe komnt, troten auoh alle drel 11lusionsatelgernden Momente auf, Aber wenn man genauer h1nsohaut, wird man elne fer unsere Zweoke wichtige Akzentrerlagemung restetelien können. E1n klein wenls Gewloht wird rom Anstreben der Gonaulgke1t weggenomen und auf elne boabsioht1gte stelgerung der Einfuhlung gelegt. Breoht erkilirt es folgendermaBen! D1. Beal1 aten hatten einen zlomlioh schematischen Helden rorgefthrt, mit "moglichst wenlgen Elnzelztgen.... dam1t or uglichst viele Zuschauer "deckte" ,28 Das helBt, deB der Held im Realismus elgentiloh unroalistischer (bzw. unnaturalistischer) als die Figuren in Naturaliamus wire. "Wenn der Zuschauer sich In Ihn einlebte, fthlte er, wie or d1. S1tuation "meisterte" .29 In a1esem vbergang rom Naturallsmus zum Real1smus (nloht chronologisch gesehen) wird also eln wenlg auf die Wahrscheinllohkelt verzlohtet zugunaten der gestelgerten Elnfthlung in den Helden. Doch was das dritte Moment (getursohte Notwendigkelt des Verlaurs) anbetrifft, hat der Realismus genau dieselbe versohlelernde W1rkung auf die Kausalitat der Geschehniafolgerung wie der

27 Heuptmann, XVI, 520.

28 Ebenda, s. 519.

29 Ebenda 
Natural1smus, und zwar aus denselben Granden (Wahrsohein110hke1t und E1nfuhlung).

Oberspringen wir nun einige Zwlschenstadien in unserem Spektrum und betracht on w1r seln anderes Ende, namlioh elnen Dramentyp, der olne stirkere Unwahrsohelnliohke1t beInhaltet, wie $z \cdot B$, einen solohen In der Ant1ke.30 Im aristotel1schen Theater, w1e es in der "Poet1k" konstatiert murde, komat das Theatererlebnis (gew1sse Katharsiswirkungen durch Furoht und Mitleld, bzw. Sohreoken und Jamer) remittelst elnes Elnfuhlungsaktes zustande. Da, wo die grenzenlose Elnfuhlung in den Helden mit Abstand die allerwloht1gste Z1elsetzung war, muBte die Illusion, man berinde sich am realen Ort, auch von hochster Intensitat sein. Diese Illusion murde zum Tell durch die Elnfanlung selbst unterstutzt, denn belde sind unlosbar inelnander verwickelt und wirken gegense1tig aufe1nander. Zum anderen Tell wurde d1ese Illusion durch das obengenannte dritte Moment geste1gert, nomlich durch die gettuschte Notwendigke1t des Verlaufs. Nun, manches im aristotelischen Theater orschien, objekt1r betrachtet, hochst unwahrsohelnlioh, z.B. In bezug auf den Inhalt der Fall, in dem in Elektra die Leute ron den pythischen Splelen berichten (Python war eln Drache). Arlstoteles behauptet in seiner Poet1k, man solle sowleso

30 D1ese Konst1tulerung des spektrume enthait den Nachte1l, auf starken Verelnfachungen zu boruhen, errulit aber die Funktion, far unsere Zwecke auselohend zu sein, und bekomint von daher ihren stellenwert. 
solohe unstimmigen Mythen nlaht konstruleren, "dooh wonn man es tut, dann soll es inen Ansohein von Wahrsohelnliohke1t haben, selbst wenn es unwarsoheinlioh 1st, und dann geht ean.31 An elner anderen stelle sagt er, daB, obwohl es ein Fehler se1, Unmogliches zu dichten, es in Ordnung se1. "renn sie (die poetisohe Dichtung, RF) damit ihr ziel errelcht..."32, und dieses $\mathrm{Z1el}$ ist, die Unwahrsohelnlichke1t als rahrscheinlioh vorzutusohen. EIn Belspiel fur Unwahrscheinlichkelt in der Darbietungsform ware der Chor oder die gerelinte, in Versen gesproohene sprache. Breoht velst selbst auf diese Unwahrsohelnliohke1t hin, wenn er 1m "Klelnen Organon" uber die Ant1ke sagt, "Unkorrekthe1t, selbst starke Unwahrschelnliohke1t storte (den Zuschauer In der Antike, $\mathrm{BF}$ ) wen1g oder gar niaht, sorern nur die Unkorrekthe1t eine gew1sse Kons1stenz hatte und die Unwahrsohelnlichkelt von derselben Art blieb," 33

Was $1 \mathrm{ch}$ mit diesem Spektrum sagen w11l, 1st folgendes, In allen Illuslonsdramen blelben zwel Elemente konstant, 1) d10 starke Betonung auf der Entstehung elner besonders Intensiven Einfahlung und 2) eine glaubhaft notwendige $\mathrm{Zu}$ sammenstellung der Geschehn1sse, die a) 1m aristotelisohen Drama sloh m1t der von Brecht genannten "Kons1stenz" begnig-

31 Ariatoteles, Poet1k, Hrag, Olof G180n (stuttgart, Ph111pp Reolam Jun., 1969), S. 62f.

32 Ebenda, 3. 64.

33 Hauptmann, XVI, $665 \mathrm{f}$. 
te, und die durch eine starke Elnfthlung versohlelert murde, und die b) 1m Natural1smus-Realismus als fest und unveranderl1ch "photographiert" marde, und die duroh die starke Elnfahlung eine Undurchsichtigkelt gewann. B. Brecht nennt das zwe1te Element Im neunten Absatz des "KleInen Organon" d1. "Illugion elnes zwingenden Verleufs der Geschlohte" (Hervorhebung von mir). Daraus erg1bt s1ch, daB, gleloh ob die Darbletungsform und der Inhalt dahin tendleren, wahrsoheinlioh odor unwahrsohelnlioh zu sein, das sogenannte "Theatererlebnis" mit starker Illusion und Einfthlung trotzdem zustandekomen kann. D1e entscheldenden Elemente sind d1e spezifisch poet1schen und theetralischen Mittel, die verwendet werden, um die Elnfthlung zu stelgern und um die Illusion elnes zwingenden Verlaurs der Geschlehte zu soharren.

Whrend $1 \mathrm{ch}$ bia jetzt in melner Konzeption die gethuschte Notwondigke1t der Geschehnlsfolge als drittes Moment in der stelgerung der theaterkonstitulerenden Illusion behandelt habe, werde $1 \mathrm{ch}$ nun dieses Moment als elgenstundiges festsetzen, nimlich als Illusion elnes zwingenden Verlaufs der Geschichte $(8.0$.$) oder als zwelte Illusion mit$ der zlelrorstellung, afgrund der Trennung der Illusion in "orste" (theaterkonstituierende) und "zwe1te". die Intention B. Brechts in bezug auf die Illusion begrelfiloh zu machen. D1ese Modifikation melner Konzeption dahingehend, daB 1oh jetzt von zwel Arten der Illusion spreche, hat den vortell. 
vermittelst dieser Fassung die Aufrassung Brechto nioht mur -1nbeziehen zu konnen, sondern sogar die Intention Brechts in deutlicher Welse darstellen zu können.

\section{Die zuelte Iluelon}

Die zwelte Illusion wird wederum durch die resultierende stelgerung der ersten Illusion und Elnfuhlung verstarkt, Indem der Zuschauer sozusagen von den Emotionen des Schausplelers gefangen 1st und machtlos hierhin und dorthin gezogen wird. D1es verhindert nach Brecht elne kritlsohe Haltung den Vorgangen gegendber und damit auoh die Erkenntn1s, daB der Verlauf der Gesohiohte abinderbar 1st. Das h1eBer Der Zuschauer ware nioht 1mstande, d10 Kausal1tut und Folge der Vorgange in Frage zu telien und damit ihre Notwendigkelt zu bezweifeln. Hier sel kurz angemerkt, daB das oben Gesagte ftr Brecht 1m groBten MaBe angtoBig sein muB, zumal os gelner politischen Intention zuwiderlufet.

Aus dlesem Tell der Untersuohung ergeben sloh rar uncere Zwecke zwe1 wiontige Punkter 1) die Entstehung und stelgerung der erston theaterkonstitulerenden Illusion, man habe es mit der Realitat zu tun, und 2) die Entstehung o1ner zwelten Illusion elnes zwingenden Vorlaufs der Geschiohte, d1e die erste dann veretarkt. Welterhin hat die erste Illusion (zusamen mit der Elnfuhlung) wederum elne Wirkung auf die zwolte, indem sle duroh d1e Verhinderung elner krit1sohen Haltung im Zusohauer elnen versohlelernden Effext In bezug auf d1e Kausalltit der Vorgunge hat, eine verschle1- 
erung, die die zweite Illusion sozusagen in sicherhe1t bringt.

B. Brechts Intention hinglohtlioh der Illusion

An den eben Erlauterten setzt nun B. Brechts Krit1k e1n, denn er wollte die (erste) Illusion und damit zugleloh auch d1e Einfuhlung begrenzen, um das Denken des Zusohauers frel zu setzen und zu aktivieren, um elnen Kritiziamus im Zuschauer zu erzeugen, den dleser dann auf selne elgene ge- lischaftilche situation verwenden sollte. Um elnen solchen Kritiziamus zu ermutigen, whe es notig, elne gewisse D1stanz des Zuschauers gegentber der Handlung zu schaffen. D1eser Abstand bedeutet elne Begrenzung der Elnfuhlung und Realitatahnliohkelt und die Ausschaltung der zwelten Illusion, nimlich der getlusohten Notwendigkelt des Verlaurs der Gesohichte.

Es 1st ke1neswogs wahr, daB Brecht mir die Natural1sten, Bealiaten und alten Griechen kritisiert hat, or nahm gegen jedes Theater, das zu viel Illusion erzeugt, elne ablehnende stelle eln. Er nennt alle 1llusionsanstrebenden Theaterversuche "Elnfuhlungs-, Fiktions- oder Erlebniadramat1 $\mathrm{x}^{-34}$ und vergle1cht sie mit elnem Karussell. "Am besten whlen wir olnes jener weltlufigen Karusselle, die una auf holzermen Bossen oder Autos oder plugzeugen an allerhand auf die wande gemalten Darstellungen von Gebirgs34 Hauptmann, XVI, 542 . 
landschaften vorbbertragen. Wir konnen auoh elnes finden, das uns in f1ktive gefuhrliohe Umgebungen sohleppt. F1kt1verwelse relten, fliegen, ateuern w1r selber...D1e Sensatlonen wechselni Wir haben elnerselts das Gefthl, ron dem Mechanl smus unwelgerlich mit gerissen zu werden.... andrerselts die Fiktion, selber zu dirigieren." 35

Damit will Brecht sagen, der Zuschauer gebe sich zuerst der Illusion hin, daB or realen Ort sei, dann ferner gebe or slah infolge des Miterlebens der Illusion hin, daB er aktir sel, d, $h$, dab er selbst mitmache, daB or die situation melstere. Aber whrend der ganzen Zelt habe er auch das Gefthl, daB d1e Geschehnlase inn unhaltbar mitfortreiBen.

In "Kleinen Organon" beschrelbt er d1e Zuschauer solcher Dramat1k als Leute, die schelnen, als ob sle ron jeder Tutigkelt entbunden selen und als "solohe, mit denen etwas gemacht wird".,36 Dieses Mitfortgerissenwerden (melst unbemaBt) des Zuschauers ist es eben, was B. Breoht mit Illusion eines zwingenden Verlaufs der Geschiohte ansprioht. Ganz entgegengesetzt dell Z1el der Sohaffung elner solohen Illusion waren selne Hauptziele gesellschartiloh-okonomisohe Z1ele, das helBt, Erat duroh die Erkenntnis, die er dem Zusohauer ermoglichen w1ll, daB d1e Dinge und Verhaltnisse verknderbar olnd, lat os, wie er melnt, dann auoh moglioh,

\footnotetext{
35 Hauptmann, XVI, 5416 .

36 Ebende, S. 674 .
} 
aur die Dinge und Verhiltnisse selbst EinfluB zu nehmen in der Form des aktiven Verunderns.

D1e zwelte Illusion kann nioht zustandekommen ohne die erste da sie jedoch die erste veratlukt, erreloht Brecht in selner 210lsetzung, de zwelte Illusion aususohalten, auch zuglelch elne Verminderung der eraten Illusion.

Be1splelhaft fur Brechts Methodik zur Ausschaltung der zwelten Illusion sind selne Erlaterungen 10 67. Absatz des "Klo1nen Organon", "D1e Geschohnlsse durfen s1ch nioht unmerklich rolgen, sondern man muB mit dem Urtell dazwlsohen kommen kolnnen." Eben jene "zwingende", und damit unbezwe1felte, oder auch rersohlelerte Kausalitut (In diesem Fall etwa d1e Szenenabrolge) als eln Haupticonstituens der zwe1ten Illusion w1ll Breoht "aufbrechen". und, Indem er so die Bandlungsabrolge mit Reflexionsknoten 37 versieht, beseltigt or damit zum Te1l auch de zwe1te Illuelon. Wle B. Breoht d10se Illusion beseltigt, wird splter ganz asfunriloh behandelt, Vorilurig aber gentigt dleses Belapiel, un uns eine einloltende Ahnung daron zu versohaffen.

Um 08 noch olnmal zu betonen, Diese Versohledenhelt der Illusionen 1st sehr wlohtig, un B. Brechts Angriff gegen die Illusion zu verstehen. S10 lat ort tbersenen wordon.

37 Hauptmann, XVI, 694. 
Elnige aufgetretene M1Breretindinisse beztig71ch B. Breohts Aurressung der Illudion. dex Elnfahluns und der Gefahle

Es gibt elne ganze Re1he von M1Bverstundnissen un die Breohtschen Theorien, besonders in bezug auf die stellung der Gerthle aberhaupt. Es lot oin glemiloh verbreiteter Glaube gewesen, daB Breont die Emotionen abschaff on wollte, samt der Elnfthlung und "Illugion", um das "kalte" Denken 1ne Theater einzurthren.

D1eses erwelst sich als Irrtum. Brecht versuchte das M1Breratundnis zu korrigleren, 1ndem er sagtel "Das episohe Theater beklmpet nicht die Emotionen, sondern unteraucht sie und moht nloht halt bel ihrer Erzeugung. Der Trennung ron Vernunft und Gerthl maoht sioh das durohsohnittliche Theater sohuldig. Inden os die Vermunft prairtisoh ausmerzt. Selne Verfeohter sohrelen belm geringsten Vermuch, etwas Vernunft in die Theaterpraxis zu bringen, man wolle die Gefunle ausrotten." 38 Brecht verbrachte verhaltnismb1g viel Ze1t mit der Aufgabe, seine Kritiker daron zu uberzeugen, dab or niohts gegen Gerahle habe. "Uns arangen die Gerthle zur auBersten Anspannung der Vernunft und die Vernunft re1nigt unsere Gerahle", sagt er in selnen Sohriften.39 Die 21tate sind zahlreioh. In Mensingkauf sagt er, "Oh, 1oh habe niohts gegen Gerthle. Ich atimme zu, daB Gerthle notig sind, demlt Darstellungen, Neohahmungen ron Vorrulien aus

38 Kuthe Bul10ke-We1ler, D1e Dramaturste Brechte (Berlin, Bensohelverlag, 1966), 3.51 .

39 Ebenda, s. 53. 
den menschlichen Zusammenleben zustande komen konnen, und daB d1e Nachahmungen Gerahle erregen massen." 40

Kathe Ballake melnt, daB eeine "Ablehnung der Elnfuhlung...v1el m1Brerstanden und fohlinterpretiert worden" sel, weil viele von seinen Kritikern eine undialektisohe Betrachtungewe1se des Erikenntnisprozesses gehabt hitton. sie setzten Elnfunlung glelch Emotionen und Denken glelch BemuBtsein, "obwohl oplteatens se1t Lenin, desaen Werke zu Breohts Handb1bliothek gehorton, klar 1st, daB omotionale (sinnl1che) und rationale (logisohe) Erkenntn1s zwe1 sturen in e1nem elnheltilchen ProzeB sind, daB die Erkenntnis nur als dialokt1sohe Elnhelt ron S1nnlichen (lebendige Anschauung) und Fationalem (Donken) existiert", 41

In Inren Ausfahrungen sagt $K$. Bllioke, daB Breoht die Emotionen nioht absohaffen wollte, doch wohl die Einfahlung. Whrend 1oh dem zuerst Genannton zustimme, so mus loh doch das Letztere vernelnen. Naturlioh wollte Breoht die Einruhlung begrenzen und hielt sle far nioht sohr fruohtbar, 1I JbermaB sogar fur wohlooht. Das MLBverstundnts kam zum Te1l wegen el nes etras ubertrelbenden Argumentationset1ls zustande, der in selnen Sohrirten mahrnehmbar 1at, und der hochstwahrsohelnlioh die Wirkung seiner unterlegenon kampelage war. Er hatte tatakchlioh elne zahlrelohe und allohtige Opposition zu tberzeugen. Des ruhrte anfangs zu olner zien-

$$
\begin{aligned}
& 40 \text { Hauptmann, XVI, } 506 . \\
& 41 \text { Btlloke-Weller, s. } 51 .
\end{aligned}
$$


lioh starken Polemik, ontw1ckelte oloh abor allmbhlioh in selnen splteren Jahren gläoklicherwel se zu elner praziseren Ausdruckewe1se.

DaB or die totale Aussohaltung der Elnfthlung nicht fur unbedingt notwendig hielt, HuBert sich in Messingkeur. "Ioh kann mir Elnfthlung als Grenzfall rorstellen, ohne daB Sohaden geachleht. Durch elne Relhe von Vorkehrungen konnte man schad on vermeiden. Sie mabte unterbrochen werden und mur an bestimmten stelion stohen, oder ganz, ganz schwach sein und gemischt mit krlftigen anderen Operationen...Wenn loh sloher sein konnte, das inr den ungeheuren Untersohied zwischen dem neuen Splel und dem alten, das auf roller Einruhlung beruht, als kaum wenlger ungeheuer sehen konntet, wenn 1ch ganz schwaohe Elnfahlung fur moglioh erklare, dann whrde 1 oh es tun." 42 Diese voralohtige Erlaubnis zelgt elne allmahliche Lookerung seiner Polemik.

Eric Bentloy behauptet mit reniger Vorsicht, aber mit Beoht, glaube 1ch, "Da Breoht unverme1dich in polenische Auseinandersetzungen verwiokelt wurde, muBte er die negative Selte dieser Inszenlerungsart aberbetonen, die ratsache, daB sle die Illusion der W1rkllohkelt zuniohte macht. Und selne Kritiker, die Immer berelt sind, zu behaupten, das eplsche Theater verleugne die Grundprinzipien des west11chen Dramas, rinden hier elnen groBen Steln des AnstoBes. S10 haben, denke 1oh, recht, wenn sie die Illusion als zu42 Hauptinann, XVI, 584. 
gehorig zur Schausplelkungt und som1t zum ganzen Theater betrachton. Unreoht haben sie nur, wenn sie annohmon, dab der erzahlende Real1st (Brecht, BF) die Illusion ganz und gar aussohaltet. Der Grad der Illusion 1 st ausschlaggebend, und ein geringerer Grad von Illusion muB nioht unbedingt weniger dramatioch sein als ein hoherer."43

E. Bentley steht nicht allein mlt dieser Aufrasaung, denn Joser Jusowsk1 z.B. sohre1bt, "Breoht konnte dem Jen1gen, der" eine volle Illusion in Theater haben w1ll, "entgegnen, daB sogar der emprangliohste Zusohauer, sowle or sich nicht der Illusion ergibt, sioh daraber klar wird, daB es Illusion 1st. Denn obgleloh dieser Zusohauer ersohtttert wird, well othello Desdemon und dann sioh selbst ermordet, wird er dooh nlcht so ergriffen sein, daB er sioh mundert oder gar gekrinkt 1st, wenn die belden Toten... aufstehen und sioh vor dem Publikum verbeugen." $4 / 4$ Diese Fahigke1t des Zuschauers, gagt Jusowsk1, "sich der Illusion hinzugeben und glelchzeltig zu wisen, daB es Illusion ist" benutze Brecht. 45

Hier wird deutlich, daB Bentley und Jusowsicl mit dem glelohen Illusionsbegriff operieren, wie loh anfangs (in

43 Erio Bentley, "D1e Theaterkunst Brechts," in S1nn und Forg (Berlini Ratten und Loening Vorlag, 1957), s. 164.

44 Joser Jusowsk1, "Bertolt Brecht und sein "Guter Mensoh" ", in Sinn und Form (Berlin, Butten und Loening Verlag, 1957), s. 208 .

45 Ebenda, s. 209. 
melner Konzeption) die Illusion charakterisiert und diese spater theaterkonstitulerende Illusion genannt habe.

Breoht selbst sagte endich in einem Gesprich mit Ernst Schumacher, "Das Lampion der Illusion, der groBe Mond der Tausohung missen leuchten! Ich bin ja nioht gegen den schonen Soheln, in den alle W1rklichke1t aur dem Theater 1mmer geraten muB. Aber weder der Sohauspleler noch das Publikum sollten vergessen, daB der Zauber, der maglsche Schein zur Durchleuchtung der wirkliohen Welt dienen muB. "46 wir werden bald sehen, durch welche Mittel Brecht dieses "Lampion der Illusion" begrenzt hat.

46 Helge Hultberg. D1e usthetisohen Anschauungen Bertolt Breehts (Munksgaerd und Kopenhagen! Kunksgaerd International Booksellers and Publishers, Ltd., 1962), s, 182. 


\section{VERFREMDUNG}

\section{EINLEITUNG}

Eln fur die Brecht-Forechung unentbehrlicher Begriff 1st die sogenannte "Verfremdung". Bevor $1 \mathrm{ch}$ weiter in me1ner Untersuchung der Stellungenahme Brechts gegentber der Illuaion weiter gehe, liegt zunlohst die Aufgabe ror, diesen Begriff der Verfrendung, so wie Brecht inn aufraBt, zu behandeln.

Ganz Im allgemeinen bezleht sich die Verfremdung auf das Verhaltnis zwischen einem vertrauten objekt und seinem Betrachter und bedeutet fur den Betrachter einen plotz11chen Verlust an Selbatreratandlichke1t in Hinbliok auf das Wesen dieses Objekta. Eln gewohnter Gegenstand vermandelt aloh in einen ungewohnten. Ob tatsdohlioh oder nur in der Subjekt1vitat des Betrachters, verliert dieser Gegenstand aus 1 rgendwelohen Grinden geine blaherige Bedeutung (bzw. gerinnt elne neue).

E1n von Brecht gegebenes Belspiel der Verfrendung 1 m taglichen Leben wire durch die folgende Frage hervorgeruf on: "Hagt du dir echon elnnal delne Uhr genau angesehen? Der mich das fragt, we1B, dab $1 \mathrm{ch}$ sie sohon oft angesehen habe, nun, mit selner Prage, entzieht er m1r den gewohnten, daher m1r nichts mehr sagenden Anblick. Ioh sah sio an, un die Zelt festzustelion, nun stelle $1 \mathrm{ch}$, auf elndringliohe Art 
berragt, rest, daB 1 oh die Uhr selber nicht mehr elnes ataunenden Bl1ckes gewtrdigt habe, sie 1st nach vielen Riohtungen hin oin erstauniloher Mechan1smus. "47

D1e Verfremdung gewinnt eine groBe Wichtigkeit fur Breoht aufgrund der positiven selte dieses Phlnomens, nim11oh der neuen Erkenntnis, die daraus result1ert, "D10 W1senschaftier machen das selt langer 2 e1t, wenn ale bestimmte Erschelnungen...betraohten und der Betrachtung zufuhren. Um das D1ng zu begrelfen, tun s1e, als begriffen sie es nicht, un Gesetze zu entdeoken, bringen ale die Vorgunge in Gegensatz zu aberkommenen Vorstellungen! dadurch arbelten sie das Krasse. Besondere der eben atudierten Erschelnung heraus. Gewisse Selbatrerstandlichkelten werden so nioht selbstrerstandlich, frellich nur, un nun w1rklioh verstand 11 oh zu werden. 48

Hier in dieam Belsplel erhalt die Verfremdung eine dem Betrachter bemuBte Punktion, d.h. s1e lst fur bestimmte Zweoke aus Ihrer Zural11gkelt herausgehoben worden. In diesen Sinne der beabsiohtigten Funktion der Verfremdung verwendet Brecht den Ausdruck "Verfremdungsefrekt" oder die Ablurzung "V-Erfekt".

Im "Kleinen Organon" definiert Breoht, "E1ne verfrendende Abbildung 1 st elne solohe, die den Gegenstand zwar er-
47 Hauptmann, XV. 355f.
48 Ebonda, S. 362 . 
kennen, Inn aber doch zuglelch rremd ersoheinen 1aBt."49 Der selbatverstundliche, alltagliche Gegenstand "wird in gew1sser Welse unveratindlioh gemacht, das geschieht aber nur, um Ihn] dann un so verstandlicher zu machen." 50 Bezogen auf die Dramatik konnte die Verfremdung nooh der Meinung Breohts auch zum Vortell dieses Verstandliohmachens verwendet werden, nimlich zum Vortell elnes fur den Zuschauer neu gewonnenen Veratindnisses selner gesellsohaftlichen Zustand11ohke1t. Der V-Effekt virde elnen Krit121smus des Zusohauers gegentber der Auffthrung erzeugen, und zwar einen doppelten. D1e Krit1k des Zuschauers 1n Brechtsohen Theater betreffe 1) die Darstellung des Sohausplelera, d.h. selne Kunst und 2) "d1e Welt, die er deratellt (s011 g1e so ble1ben?) *.51 Brecht meint allexdings, man habe auoh in ab11chen KunstgenuB elne hohere stufe, die krit1ech genleBe, -aber d1e Krit1k betrifft hier nur das Art1 tisohes den segenuber ist es etwas ganz anderes, wenn nicht die kanstlerlsche Darstellung der Welt, sondern de Welt selber kr1t1 sch, widersprechend, distanzierend betrachtet werden mol1".52 Er melnte velter, daB es im "alten" Theater wohl auch Verfremdungen gegeben habe, aber s1e klmen hauptalloh$11 \mathrm{ch}$ da vor, wo "Fehler" gemacht worden selen. "Wenn die

49 Heuptmann, XVI, 680.

50 Ebenda, XV, 355.

51 Ebenda, s. 377 .

52 Ebenda, s. 378. 
Perloke des KOniga Lear sich verschiebt, tritt zutage, daB da nicht Lear etirbt, oondern der Sohauspleler Meyer, und der et1rbt naturiloh nicht. Seine "Absiont", uns zu tursohen, 1st entdeckt rorden, w1r sind bose, nioht we11 or uns tluschen wollte, sondern we11 or es nioht konnte." 53

Der Natural1amus trefre af Kritik, wenn er "die IIlusion nicht herstelien" konne. "Es goht ouoh (den Naturalisten, RF)" w1e Breoht im Kegsingirauf sagt, "wie dem Hypnot1 eeur, dem d1e Hypnose miBl1ngt. Dann kr1t1siert der kl1ent cen Aprel, der eine zitrone 1etim54

Bel aleser in d1esen Fall zufuligen Verfremdung 1 at far Brecht mur die eine selte der Kritik vorhanden. D1e Frage bezlont alch nur aur das Abb12d, aber nioht auf das Abgebildete.

E1ne reltere, vorher erwahnte, von Breoht auf den Naturaligmus gerichtete Kritik beruht sowieso auf der photographischen W1edergabe der W1rkl1chke1t. "Vorgunge und Peraonen des Alltags, der umittelbaren Ungebung, haben Ifir uns etwas Natarliches, well Gewohntes. Ihre Verfremdung dient dazu, a1e uns aufa111в zu machon."55 Doch dem Naturalisten kime es gerade "auf die Natarliohkelt an, und so sohion alles bel $1 \mathrm{hm}$ viel zu nattriloh, als daB man sioh dabel aufgehalten hatte, es elgens zu untersuohen".56 Man

53 Hauptmann, XV, 363.

54 Ebenda, XVI, 521.

55 Ebenda, XV, 347.

56 Ebenda, XVI, 516. 
untersuche je fur gewöhnlioh auch nlcht selne elgene Wohnung oder seine EBsitten.

Daraus wird es in bezug auf den Naturalismus nach Brecht doutlich, daB, wenn elne auf eln naturalist1sches Stack gerlchtete Kritik Im Zuscheuer zustandekome, sio nicht durch das stuck selbst beabsichtigt murde, und daB sie nach Brecht nur die ktnstlerische Selte der Daratelluns betrifft, nlemals die Gesellschart, ron der das Abb1ld gemacht wurde.

\section{HAUPIGKEIT UND TBADITION DER KONSTLERISCHEN} VERFREMDUNG UND B. BRECHTS ABGRENZUNG DAVON

Es muB hier gesagt werden, daB der Begriff der Verfremdung kein neuze1tlicher 1st. W1e loh sohon in meiner Behandlung der theaterkonet1tulerenden Ilusion zu zelgen versucht habe, 81 bt es versohiedene 11lusionseinsohrankende M1ttel, d1e benutzt werien, un das Drama (besser gesagt, d1e Kunst aberhaupt) Innerhalb des Berelchs des Asthet1schen zu behalten, Indem es gerade die restlose Illusion im sinne ron totaler rausohung vermeldet.

D1e Ant1ke und andere Dramatiken lieBen die Sprache durch Vers fremd erscheinen. Masken, Chore, Mus1k und v1ele andere real1titafrende Formen haben sioh in die Theatertradition elngebettet.

Brecht wirde dies alles zugestehen und nonnt selbst zahlrelche Kunstler (Innerhalb und auBerhalb des Theaters). die die verschiedensten verfremdenden Methoden angewandt 
haben. James Joyce $z \cdot B$, verfremde sowohl "dle Darstellungsart (hauptsilchlich durch hlufiges und schnelles Wechseln) als auch die Vorgange".57 Cezanne verfremde in selner Kalere1, wenn er "dle Hohlform elnes GeraBes uberbetont. Der Dadalsmus und der Surrealismus benutzen Verfremdungseffekte extremster Art. Ihre Gegenstinde kehren aus der verfremdung nloht wleder zurllck,"58 "Wenn in Granewalds Altarbild der Evangelist selber ins Bild gestellt 2st, wird die Kreuzigung verfremdet."59 Auch die ostasiatische Sohausplelkunst habe schon lange V-Effekte gebraucht, besonders in den st111sierten Gesten. Als Vorbllder der Verfremdung nennt Brecht StraBenverktufer und Z1rkusolowns.

Reinhold Grimm in Kom1k und Verfremdung behauptet sogar, daB d1e Kom1k berhaupt elne Verfrendung se1, und ex wird eine weltgehende Unterstutzung ron Helmut Amtzen 60 bekommen. Brecht selbst sagt, "Allgemein angerendet wird der V-Effekt in der Kombdie, besonders der nledrigen. Kehrt eIn Ehemann spt in das chellche Schlafgemaoh zurtol und belausoht $1 \mathrm{hn}$ die vollig wache Gattin belm Herelnschlelohen, so wird dieses zur Ergotzung des Publikums verfremdet." 61 Es gibt noch viele Belapiele. Es lohnt sloh aber

57 Hauptmann, XV, 364.

58 Ebende

59 Ebenda, s. 367.

60 Helmut Arntzen, "Kombd1e und ep1sohes Theater," Deutschunterrioht, Jg. 21, Hert 3 (Jul1 1969), S. 67-77.

61 Heuptmann, XV, 366. 
nlcht, alle hler aufuzahlen, da diese sohon ausrelohend genug sind, die Hafigkelt und Tradition der kanstlerischen Verfremdung aufzuze1gen.

Brecht sah den Wert dieser Mittel, Indem sle sicher11ch die Elnrthlung verhinderten. Aber bezogen auf das Theater melnte or zu erkennen, daB $1 \mathrm{hr}$ Gebrauch zum grobten Tell durch den technischen Fortschrltt des Theatralischen ausgemerzt worden sel. In der Ant1ke, bel Shakespeare und in der deutschen Klassik nimlich war das Theater noch nicht 1mstande, die sinnliche W1rkl1chke1t effektir nochzuahmen, we es die Naturalisten getan haben. ober das Theater der deutschen Klassik z,B. sagt Brecht, "Lle Llohterfekte waren nooh primitiv, denn es gab die elektrische Blrne noch nicht. Wo mangelhafter Geschmaok die Abendsonne zul1eB, verhinderte mangelhafte Maschinerle die voli1ge Berdokung.... zumindest wo es bel der Tauschung versagt, zalgte sich das Theater noch als Theater. .62

Was d1e Verfremdung anbetriff, will Brecht uns ze1gen, dab der Naturalismus die allerletzte ungleckliche sture elnes Frozesses sel, In dem dieses Mittel allmahlich aus dem Theater hinausgeschoben worden sel zugunsten der restlosen Illualon, man befinde sloh am roalen ort.

Hier 1st viellelcht eine geelgnete Stelle, Brecht hinsichtlich selner Kritik am Naturalismus selber anzugro1fon. Brecht streltet dem Natural1 mus jegliohe Hoglichkelt

62 Hauptmann, XV, 251. 
des Reflektlerens des Zuschauers aber das Dargestellte ab m1t der Begrundung, das naturalistische Vorgehen warde in starkster Welse eine Elnfuhlung des Zuschauers in die auf der Buhne dargostellten F1guren produzieren loder ermogl1chen). die damit den Zuschauer in elne rollkommene Unfre1he1t des Denkens versetzen warde. Elnerse1ts 1st dies fur mich oin AnstoB der Kritik, daB Brecht meiner Ansicht nach auf zu polemische Welse die Naturalisten in inrer gesellschaftlichen Wirkung kritisiert, da es nooh lungst nicht bewlesen worden 1st, daB es im natural1st1schen Theater unmbglich 1st, elnen Kritiz1smus Im Zuschauer ontwickeln zu konnen. Es 1st zunindest denkbar, daB auch ein Zuschauer des natural1stischen Dramas (gerade wenn dieaes Drama $z . B$. ein politisches 1st). zur Reflexion uber das dargestelite gesellschaftliche system trotz der zugegobenen relativ atarken EInfuhlung angeregt wird, ganz abgesehen davon, das Brecht dem Zuschauer in zu geringem KaBe ein elgenes selbatundiges Denkrormbgen unterstelit und inn zu sehr in einer Abhanglgke1t vom Dargesteliten gebunden sieht.

Andererse1ts sehe $1 \mathrm{ch}$ es als fragwardig an, daB Breoht behauptet, die Naturalisten warden elno restiose IIlusion daningehend, daB es sich auf der Bahne un dio Wirkliohke1t selbat handle, erzeugen. Es 1st zwelfellos richt1g, daB das naturalistisohe Theater olne starke Illuaion zu erzeugen vermochte. Dooh tut man gut daran, auch hier die Grenzen dieser IIlusion zu bemerken. Sogar die Naturalisten 
haben, trotz 1hres Anstrebens, die opt1sche W1rklichkelt nachzuahmen, den Rahmen der Bthne belbehalten, durch dessen Storung der Realitatsahnlichielt de Illusion bereits begrenzt w1rd. Wenn auch der Naturalismus elne "photograph1sche" Wledergabe llefern wollte, so hat schon das Photo selnen das B1ld von der Realit tabgrenzenden Rahmen.

Dies soll nicht den Anschein erweoken, als ob 1ch mit dieser Anmerkung Brecht in grundlegender, well seine Intention betreffender, Hinsicht kritisleren wollte. Ganz das Gegentell 1st der Fall, da loh namlich Brechts Richtung se1ner Ausfahrungen zugt1me. Eg kam mir lediglich darauf an, den hlex ron $1 \mathrm{hm}$ vorgelegten polemischen Akzent innerhalb selner Kritik an den Naturalisten herauszustrelohen.

Es darfte gefragt werden, warum 1 oh in dlesem Zusammenhang den V-Effekt im nichtbrechtschen Theater erortert habe. Diese Behandlung I1ndet, glaube 1oh, 1hre Berecht1gung nioht nur als Hinwels auf die Tatsache, dab der VEffekt in bezug auf die Begrenzung der theaterkonstitulerenden Illusion bel Brecht nicht erst- und einmalis 1st, sondern auch als Wegwelser zum Verstandnis von Breohts umfassenderen und von ihm kritisoh begrtindeteren verwendung des V-Effekts im Hinbliok auf seine spezifisohe Wirkungsintention, die eine fur die Brecht-Forsohung wichtige Differenzlerung innerhalb des Begriffs involviert.

Der Gebrauch des V-Effekts in ablichen Theater beruht nomlich vorwiegend nur auf der Begrenzung der theaterkon- 
st1 tulerenden Illusion, Indem er aus rein lsthetisohen Granden die Dinge und Verhaltnisse des realen Lobens auf der Buhne fremd erscheinen 1aBt. Insorem das Theater zu einom gewlssen Grad diese Verfremdungen gebraucht, um immer nooh als "Theater" sich realisieren zu können (und das helBt innerhalb des Rahmens des Asthet1schen ble1ben zu konnen). fande loh es legitim, dieses Mittel der Begrenzung der theaterkonstitulerenden Illusion selbst als theater konstitulerend zu nennen.

D1ese theaterkonst1tulerende Verfremdung hat an $81 \mathrm{ch}$ wenis mit Brechts gesellsohaftsverandernden Wirkungsintention zu tun, oder besser gesagt, in nur indirekter welse. III gewohnlichen Theater manifestiert sloh der V-Effekt als rein kthstler1sches Mittel. De aber Brechts 21ele tber den Bereloh der Kunst b1s in den Bereloh des Politischen hinausgehen, hat er fur den V-Effekt elne neue Begrindung gefunden, deren Form sich zwar ktinstlerisch auBern leBt, aber deren Funktion gesellsohaftlioher Art 1st.

Brecht verwendet allerdings diese theaterkonstitulerende Verfremdung, da elne zu starke theaterkonstitulerende Illusion, einsohlieBlich der begleltenden Einfahlung, elner Verschlelerung der Kausalitat der Vorgange dient. Infolgedeasen war es, so wie Brecht meinte, um den Kausalkomplex der Gesohehnisse zu entlarven, zuerst notwend18, der theaterkonst1tulerenden Illusion 1hre verhullende starke zu entziehen. Daduroh gewinnt der von m1r genannte theaterkon- 
stituierende V-Efrekt elnen wichtigen stellenwert in der Theorle und Kunst Bertolt Breohts.

Dleser von Breoht begrandete Doppelfunktion des VEffekts entspreohend, die letzten Endes auf e1ne Veranderung der Gesellschart abgezlelt 1st, untersohelde ich zw1schen zwe1 Verfremdungsarten, oder besser gesagt, zwischen zwe1 verschledenen Stufon in einem elnheltilohen VerfremdungsprozeB, D1e erste verfremdungsart entspricht der Begrenzung der theaterkonstitulerenden Illusion, und die zwe1te, der unmittelbaren Beseltigung der Illusion elnes zwingenden Verlauf der Geschlohte.

Aber beror loh mit elner tiefgehenderen Gllederung dleses Verfremdungsprozesses fortfahre, moohte $1 \mathrm{ch}$ zunlohst ein par aligemelne Erluterungen zu der Verfremdung in Brechtsohen Theater goben.

\section{VERPREMDUNG BEI BRECHT IM HIN-} BLICX AUF THEATRALISCHE MITTEL

Obwohl diese Untersuchung slch rorwlegend auf des Produkt des Stuckeschrelbers konzentriert, nlmlioh auf den geschrlebenen Text, betreffon die Formen und Mittel des V-Effekts alle Produzenten der Sohausplelkunst, auch Sohauapleler, Bthnenbildner, sogar Musiker und Choreographen (der Beg1sseur wird nattirlioh auch intensiv am Theaterprodukt betelifgt seln, aber alne Lelatungen auBern sioh nur mittelbar durah Sohauspleler, Bthnenbildner, Musiker, Choreogra- 
phen usw.). Da Brecht diese schon austhrlich genug in se1nen Schriften behandelt, und da sie uns in dieser Untersuohung nur indirekt interessieren, lohnt os sich nioht, slch lange dabel aurzuhalten. Doch konnten elnige beschre1bende, zusammengezogene Bemerkungen letztlioh dazu dienen, unser Gesamtbild des Brechtschen V-Effokts ein wenig aufzurunden. Daher sel folgendes gesagt.

\section{Schauspleler}

Der Schauspeler darf sich nioht restlos in selne Rolle verwandeln. Er muB selne Flgur so darstellon, daB der Zuschauer alch 1mmer dessen berubt 1st, da stehe der Schauspleler und or splele olne Rolle. Dies Phanomen nennt Breoht im "Kleinen Organon" "In zwelfacher Gestalt auf der Bthne stehen".63 Das bedeutet, Der Schauspieler "ze1gt" selne Flgur, antatt sie zu "erleben". Da die Gerthle dea Publikums nicht die der Figur sein sollen (des Publikum soll an andere Moglichkelten denken kobnen), sollen selne elgenen Gerthle nicht die selner Figur seln; sle kbnnen Ihnen sogar midersprechen. Dieses "Ze1gen" setzt voraus, daB die "Bezlehung des Schauspielers zu selnem Publikum...die allerfreieste und direkteste" sein muB.64 Er zelgt seine F1gur dem Zusohauer, und das bedeutet, daB er mit den Zusohauern kommuniziert. Er kann sich sogar direkt an das Publi-

\footnotetext{
63 Hauptmann, XVI, 683.

64 Ebenda, XV, 406.
} 
kum wenden und $\mathrm{zu}$ ihm reden.

Die situation des Schauspielers soll nloht als ein spontanes, erst jetzt entstehondes Erelgnls rorkomen, sondern als schon lange elnstudiert und getbt, damit der $z u-$ sohauer nicht mit der Versuchung konfrontiert wird, sich in die Illusion, er befinde sloh am ort eines erst- und elnma11gen Geschehn1sses, hineinzubegeben. D1es bedeutet, daB der Schauspleler selnen Dlalog "zitieren" soll. "DaB der Text nloht momentan entsteht, daB er memorlert 1st, etwas Fixiertes, braucht er nioht vergessen zu machen...Er z1t1ert elne P1gur, er 1st der Zouge bel elnem ProzeB, .65

Die Haltung des Schausplelers habe nach Brecht otwas W1dersprtohliches in sioh. Ex konne selne Flgur mit den Gerahlen ausstatten, die sie haben soll, und er konne deutileh machen, wenn de Flgur inre Worte momentan ausatoBe (er ontwlokele sogar selbst Gefunle), "aber es alnd nicht notwendig dieselben wie die der P1gur", 66 und or spreche in der "Vergangenhe1t", whirend die P1gur in der Gegenwart spreche. Wehmen wir an, die Flgur sagt etwas, was sle wahr glaubt. Der schauspleler kann ausdrtoken, muB ausdrtaken konnen, daB es unwahr 1st, oder, daB das Sagen dieser Wahrhe1t verhungnlsvoll 1st oder anderes." 6 ?

65 Heuptmann, XV, 351.

66 Ebenda, S. 352 .

67 Ebende 
Der Schauspleler verfremdet geine Plgur und zelgt zuglelch selne Xritik an den gesellsohaftlichen Implikationen dessen, was er selbst darstellt, un dasselbe rur den Zuschauer zu ermbglichen.

\section{Bahnenb1laner}

In seinen Sohriften tber Bthnenbau behauptet Brecht, manche Bahnenbildner warden Ihren Ehrgelz dareln setzen, daB man, Ihre Buhne betrachtend, glaube, an elnem w1rkilchen Ort des wirklichen Lebens zu sein. 68 Der Bthnenbilaner im Brechtschen Sinn werde hingegen von diesem ablichen Zwang befrelt, "wenn er belm Aufbau der Sohauplatze nlcht mehr die Illualon eines Baumes oder einer Gegend erzielen muB".69

Das Buhnenb1ld werde auf das Not1gate beschränt. So wie der Parabelerzunler alles, was or fir seine Parabel braucht, zelgen will, zelge der Buhnenblidner der Parabel auch die Sachen, mit deren Hilfe die Parabel gebaut werden soll. Aber was "auf der Buhne steht, muB mitsplelen, und was nicht mitspielt, muB nicht auf d1e Bunne".70 Dies erzeugt eine verfrendende Karghelt, die Brecht fur seine Buhne verwlrkl1cht sehen w1ll. Und diese Karghelt werde auoh daduroh unterstatzt, "daB der nioht1llusionistische Buhnenbau sich mit Andeutungen der Merkmale Begnigt, mit Abstrak-

68 Hauptmann, XV, 451.

69 Ebenda, XVI, 698.

70 Ebenda, XV, 453. 
tionen arbeltet, so dem Beschauer die Mhe des Konkret1alerens aufbardet. Er tritt der Lahmung und Verktmmerung der Phantas1e entgegen." 71

Das Buhnenb1ld verfremdet also dle optische W1rkl1ohkelt duroh Weglassen der Perlpherle und durch selne in Form ron Andeutungen magere Wledergabe der Dinge, die 1hrer Anresenhelt auf der Buhne dem Gltok zu danken haben, daB slo regen des Inhalts 1rgendwann elnmel, se1 os nur kurz, in den Brennpunkt kommen.

Mustker und Choreograph

Was die Theatermusik und Choreographie anbelangt, muB gesagt werden, daB dlese Mittel ron romhereln schon durch 1hre Unwahrschelnlichkelt das abgoblldete Leben verfremden. E1n sterbender Mann $2 . B$. 1st roal. Aber wenn or glelohze1t1g anfungt zu alngen, verfrendet or dieses Erelgnis. Dooh wenn die Musik benutzt wird, um auf suggest1re Welse stimmungen zu schaffen, konnte, wie wir in der Oper hulufig beobachten konnen, sie als starkes elnfuhlungsstelgerndes Werkzeug dienen. Eln groBer Tell der Musik bestehe aus diesen Ausmalungen subjeirtiver St1mmung. "Solohe Musik gestattet, da sle den Horrer emotional soglelch ansteckt und glelchstimmt, keine Beurtellung (rationaler und emotionaler Art) der betreffenden seelenstimmung selbst" 72 und ware fur das

\section{Heuptmann, XV, 453.}

72 Ebenda, s. 487 . 
Illuslonstheater daher an besten da elngesetzt, wo die Vorglinge auf der Buhne elne solche Beurtellung gestatten warden.

Deshalb sollten in Brechts Theater "die Schauspieler nioht in den Gesang 'Ubergehen', sondern $1 \mathrm{hn}$ deutlioh vom Ubrigen absetzen...D1e Musik muB sich 1hrerse1ts durchaus der Glelchsohaltung widersetzen, die lhr gemelnhin zugemutet wird und die zur gedankenlosen Dienerin herabwardigt".73 sie solle also nloht "beglelten", es sel denn mit Komment. "S1. begnige sich nicht dam1t, slch 'auszudrticken', Indem s1. sich elnfach der Stimmung entleert, die sie bel den Vorgangen beful1t."74 Die Mus1k solle sich selbstandig etablieren und in lhrer Welse zu den Themen stellung nehmen. Das bedeutet, daB die stimmung der Musik der stimmung der Buhnensituation wideraprechen konnte. Zum Belapiel in e1ner Auffuhrung von Brochts Kaukas1 schem Kre1dekre1s, könnte, an elner stelle, wo der sunger die auf der Bthne pantomimisoh dargestelite Rettung des Kindes durch die Magd besohrelbt, "elne kalte und unbewegte singwelse...die schrekken elner zelt entbibBen, in der Matterlichkelt zu selbstmorder1soher Sohwlohe werden kann".75

Und in bezug auf die Choreographle, die Brecht nur sehr lurz behandelt, sagt er in "Klelnen Organon", "Schon

73 Hauptmann, XVI, 697.

74 Ebenda

75 Ebenda 
d1e Eleganz elner Bewegung und die Anmut elner Aufstellung verf rendet. ...76

Diese kurze algemelne referierende Behandlung genugt fur jetzt, oine einleitende Verstundnisbasis des Verfremdungseffekts als Ausgangsatof zu fundamentleren. Es wird damlt klar, daB alle Schauspielkunste, Kostemsohnelden und Maskenmachen elngesohlossen, In der Absloht der Brechtschen Verfremdung zusammenarbelten, un die Illusion zu durohbrechen.

\section{VERFREMDUNGSPROZESS}

Der folgende Entrurf ist eln durch Projektion hergestellter, auf zahlrelohen Erlauterungen und Anwendungen von Breoht beslerender Versuoh, Bioh suf den V-Effekt in selner Ganzhe1t, d.h. von colnem Ansatz im Drama bis zu dem erhofften Besultat in der Gesellschaft, von Breohts Wirkungsintention auggehend zu konzentrieren, um diesen for ung zu konkret1sieren. Ob dieser VerfremdungsprozeB tatsachlich 1II Zuschauer stattfindet, d.h. sloh im Zuschauer effektiv realisiert, soll jetzt zum grobten Tell spekulation bleiben. Was uns an Brechts Verfremdungstheorien interessiert, soll sich hier auf die mogliche Realiaation der Theorie in der Praxis besohrtiken, denn die Vollziehung von Brechts Intention kann, wenn aberhaupt, nur unter den besten Voraussetzungen in Zuschauer ala sloher gelten. Dies wird uns e176 Hauptmann, XVI, 698 . 
nen zumindest klareren Einblick in das Hodurch, die dramat1sche k1ttel betreffend, In Brechts Herum, bezogen aur die geselleohart11ohe Intention, geben konnen.

Es sel an dieser Stelle geangt, daB die folgende Untersuchung vorwiegend an dom stilok Breohts Der gute Mensoh Ion Sezuen orient1ert 1at.

Verremdung bef1ndet sich gelegentlich und teliwe1se auf zwel versohiedenen Ebenen, 1) auf der Ebene des Reflexes und 2) auf der Ebene der Rerlexion.

Verfremduns auf dex Ebene des Befleres (Vorstufe)

Auf der Ebene des Reflexes wird eine verhultnismaBig gedankenlose Rerlerberegung Im Bembtsein des Zusohauers erzeugt, die thn wookt und aufmerksan macht, Der Te11 der Verfremduns, der sich auf aleser Ebene beflndet, aberrascht den Zuechaver durch olne Storung der Gerohnhe1t, durch eine D1 shermonie, etnen M1BKlang, Elne Erwartung wird enttuscht (bzw. ein Unerwartetes tauoht auf). wie otwa die plotzlicho Unterbrechung eines bis dahin flieBenden Dramas.

Auf dieser Ebene wird nooh niont reflekt1ert, denn kein sinngehalt wird hier in diesen stadiun vorfremdet. Der Te11 dea V-Effekta auf dieser Ebene betrifft elgentI1oh die Techn1k, ale selbst kelne inhaltilohe substans aurwelat. Schon bel der Elnsetzung eines solohen V-Efrekts auf der Ebene des Reflexes hat or selne Aufgabe berelts erledigt. nimlich das Aufmerksammachen und das automat1sche Len- 
ken des Augenmerks auf den begleitenden sinngehalt dieser bestimmten stelle im stfok, eln Sinngehalt, der absichtiloh von Breont durch diese "AuBerliche" Verfremdung in den Vordergrund gertokt wird. Zum Belsplel, Wenn shen Te plotzlioh mitten in der Artion sioh an das Publikum wendet und direkt zu uns spricht, werden w1r aus irgendwelchen Illusionen, wir befinden uns beim Beobachten elnes realen Geschehnisaes. gerlasen und gofort zum Bemubtaein gebracht, daB wir in e1nem Theater sitzen.77 Hierdurch wird der sinngehalt dessen, was geaggt wird, auf verfremdende welse ine Lloht gebraont. D1es tritt dann besonders stark auf, wenn in der d1rekten Anrede elne Prage ans Publikum gestellt wird. Denn h1er wird sehr deut $11 \mathrm{ch}$, daB der Zuschauer aus selner Rolle des passiven Hbrers herausgehoben wind und zur alrekten Aktivitat aufgefordert wird, denn eine Frage verlangt eine Antwort.

77 DLes xird in sturkerem MaBe zu Brechts Zelt der Fall gewesen sein, da heutzutage praxt1soh jeder relohiloh die Gelegenhe1t hat, Verfromdungen verschiedenster Art in der Kunst zu beobechten. Wenn diese nioht aliein duroh die gegenwartigen Tendenzen der Kanste vermittelt werden, die ohnehin verfrendender sind, alo sio zu Brechto Zelt waren. dann hat man sich durch das Pernsehen und K1no in aure1chendem MaBe an Verfremdendes gewbhnt. Jedenfallo versuche $1 \mathrm{ch}$ in melner Untersuchuns, uns als Zuschauer in die Schuhe der domaligen Zusohauer zu versetzen, um deutlioher ale intendierte Wirkung Brechts darzustellen. Doch 1st es melne Ansioht, daB es auch heute noch Hensohen glbt, die in BrechtBohen Theater in eohter Welse Verfrendungen erleben. Auf der anderen Selte hoffe loh, dab nienand os mir lbel nimmt. wenn er sich beim Lesen dieser Untersuchung, wie er meinen mag. falscherrelee in das kollektive "wir" hinelngezmungen sleht. 
D1esea Stadium der Verfrendung wird nicht nur durch direkte Anrede ans Publikum errelcht. Der stindige Wechsel zwischen Prosa und Vers liegt auch auf dieser Ebene. Der Vers, w1e 1oh sohon erortert habe, 1st durch seine Unnatur11ohke1t sow1eso verfremdend, aber eln stundigea Wecheeln ron einer stilebene zu der anderen muB diese W1rkung nooh veraturken. Indem w1r mit jedem Weohsel einen stob bekomen, stattdessen, daB wir kont1nuleriloh mit elnem st11 (ae1 os Vers oder Prosa) mitrlieBen konnen. Dieses Hittel wire oft mit einer direkten Anrede ane Publ1kum gekoppelt, un den Errekt noch weiter zu stelgern.

E1ne belsplelharte Stelle aus dem Guten Menschen ron Sezuen 1 st der Monolog Shen Tea bel threr Entdeckung, sie eel sohwanger. 78 D1e stelle rungt in Prosa an:

- Freudel Ein kleiner Mensch entsteht in meinem Le1be. Man sieht noch nichts. Er 1at aber schon da. Die Welt erwartet inn in gehoimen. In den stadten heiBt os schon: Jetzt kommt elner, mit dem man roohnen muB. (S10 etelit Ihren kleinen Sohn dem Publikum ror): Eln Flieger I

Von de gent ale unnittelbar in einen reinlosen Vero uber, der einen neuen hymischen Ton hat, motiviert duroh die HInrendung zum Publikum:

Begrabt einen neuen Eroberer Der unbekannten Gebirge und unerrelchbaren Gegenden! Elnen

Der d1e Post von Mensoh zu Mensoh

ober die unregeamen waten bringt!

Der Honolog wim denn intim, als sie durch eine von einer

78 Hauptmann. IV, $1568 \mathrm{f}$ 
Pantomime beglelteten Prosapasage 1hrem 1maginuren Sohn

auf spielerische We1se die Welt zelgt und mit ihm splelt,

(s1e beginnt auf und $a b$ zu gehen und 1hren kleinen Sohn an der Hand zu nehmen), Komm, Sohn, betrachte die WeIt! Hier, das ist ein Baum. Verbeuge dich, begriBe 1hn. (s1e macht die Verbeugung vor.) So, jetzt kennt Ihr euch. Horoh, dort kommt der Wasgerverkdufer. EIn Freund, glb $1 \mathrm{hm}$ dle Hand. Sel unbesorgt. "B1tte, eln Glas frisohes Wasser fur melnen Sohn. Es ist warm." (s1e glbt I him das Glas.) Aoh, der Polizist! Da machen w1r elnen Bogen. Vielleloht holon wir uns ein naar K1rschen dort, im Garten des reichen Herm Feh Pung. Da helBt es, nicht gesehen werden. Komm, Vaterloserl Auch du w1llst K1rsohenl Sachte, sachte, Sohnl (s1e gehen vorsicht1g, slch umbliokend), Nein, hier herum, da verb1rgt uns das Gestruluch. Nein, so gradlos drauf zu, das kannat du nloht machen, in diesem Fall. (Er schelnt sie wegzuziehen, sie widerstrebt,) w1r mbssen vernanftis seln. (PIotzlich gibt sie nach.) Sohon, wenn du nur gradezu drauflosgehen w1llst... (S1e hebt ihn hoch.) Kannst du d1e K1rschen errelohen? Sohleb in den Mund, dort sind sie gut aurgehoben. (S1e verspelst selber e1ne, die er $1 \mathrm{hr}$ in den Mund steokt.) Schmeckt roln. Zum Teufel, der Pollzist. Jetzt helBt es laufen. (S1e rliehen.) Da 1st die strabe. Buhig jetzt, langsax gegangen, demit wir nioht aurfallen. Als ob nicht das Geringste geschehen ware...

Ein we1terer Wechsel der Sprachrorm in gesungene Kinderre1-

me beendet den Monolog,

Elne Prlaume ohne Grund

Oberfiel 'nen Vagabund.

Doch dor Mann war auBerst quiok

B1B die Prlaume ins Genlok.

W1cht1g zu bemerken 1st, daB wir auf dieser stufe des

Prozesses noch kelne inhaltliche Verfremdungen analysieren. Was uns bel shen Tes Passage hier interessiert, 1st ledigI1ch der St1lwechsel, nloht der sprachllohe Inhalt. Dieser wird durch don sprungharten luBeren Aufbau allerdings in den Folus geschoben, aber noch nioht verfremdet, da eine Bearbeltung des gelstigen Storfes die Reflexion beschaftigt 
und schon zur nlohsten stufe gehort.

Dem AuBeren Aufbau tberhaupt wird von Brecht elne s1oh auf der Beflexebene befindende, verfremdende Funktion gegoben. Der gute Mensoh ron Sezuan besteht aus zohn Szenon, einem Vorsplel, elnem Ep1log, eleben Zw1schensplelen und mehreren Songs, die dberall in das Stllok elngesohoben worden sind. Vom unmittelbaren Eindruok dieser gesohuftigen, Begmentierten, hin und her zlehenden, offenen Form kommen wir nloht zu der Buhe, zu der wir bel elnem geradilnigen, ze1t- und ramilch, handlungamaBig elnheltilohen, geschlogsenen Drama kommen wirden. Oft ersoheint ein solches unterbreohendes Moment, wie $z \cdot B$, oln kurzes Zw1schenspiel vor dem Vorhang, zusammen mit elnem St1lwechsel und elner direkten H1nwendung zum Publikum (Shen Tes erstes Zwischenap1el). Unter diesen Umstanden falt es dem Zuschauer aberst sohwer, elne starke Illuslonlerung zu erleben. Breoht sagt 1m 67. Absatz des "Kleinen Organon", "Da das Publikum ja nicht elngeladen werde, sich in die Fabel wie in einen FluB zu werfen, un sich hierhin und dorthin unbest1mint trelben zu lassen, mossen die elnzelnen Geschehnisse so verimbet seln, daB die knoten auflulilg werden. D1. Geschehnisse durfen sich nloht unmerklich folgen, sondern man muB m1t dem Urtell dazw1schenkommen können." Und 1in vierton Nachtrag zur Theorie des Hessingkaufe he1Bt os In bezug auf diese Diskontinulerlichke1t, "Stundig zieht diese splelwelse alle Kraft aus dem Vergleloh mit der Wirk- 
11chke1t, das he1Bt, sle lenkt das Auge stundig auf die Kausalltat der abgebildeten Vorgange."79 D1e Zwisohensplele Im Guten Mensohen 2.B. runktionleren als diese aurfallenden "Knoten" oder Unterbrechungen in der Handlung, die den $\mathrm{Zu}$ schauer aufmerksam machen und $1 \mathrm{hm}$ die Moglichkelt geben, tber die Handlungen nachzudenken.

AuBer den direkten Anreden an das Publikum und dem AuBeren Aufbau (St1lweohsel gehort atreng genommen auch hierhin) gibt es noch elne weltere Verfremdung auf der Ebene des Reflexes, nümlich den Klang der Sprache, Unter dieser Verfremdung verstehe $1 \mathrm{ch}$ ungewdhnllohe Konsonant- und VokalKombinationen und -Wiederholungen (Alliteration, Relm, usw.), unnatdrlichen sprachrhythmus und -akzent, und unnatarliohe Satzmelodie (Tonberegung), Vers und song fallen automatisch in diese Verfremdungskategorle hinein, je ausgeprater die Form, desto verfremdender sind sie. Aber Prosa (die rea11stischste Sprache) lann auch verfremdend sein, wenn eine Klangkombination plotzlich auftaucht, die unsore sprachgewohnhelt stort, wie $z, B$. eln zufalliges Reimen.

Wenn d1e Frau in der ersten Szene des Guten Mensohen sagt, "Je sohlechter es ging, desto mehr murden wir. Ind je mehr wir wurden, desto schlechter ging es", 80 werden w1r, abgesehen vom Inhalt zunfohst, vor lauter klangliohen Wiederholungen auf verfremdende We1se "1rritiert". Wenn

79 Hauptmann, XVI, 655 .

80 Ebenda, IV, 1506. 
w1 diese satze analysieren, dam sehen wr, daB alle WOrter auber elnem genau zwelmal ereoheinen. Das elnelge, was sioh niont wiederholt, int das Wort "Und" und dieses erwelst aloh als Drehpunkt der ganzen, fast parabolisohen Tonkonbination.

H1er, wie bel all diesen V-Effekten auf der Fbene dea Rerlexes, bekomt der Zuechauer einen "tuBeren" autmerkaammachenden StoB, der den Weg fur den begleltenden Inhalt frel maoht, so deB dieaer in den Vordergrund au rioken vermag. D1eser Vorgeng iat in Frine1p nioht andera, ala wenn elner mit der Absioht, elnem anderon etras zu sagen, von hinten aur dio Sohulter dieves klopft, un zuerat selne Aurnerkeamke1t zu gowinnen. Nochden der andere in einen aufmexksamen Zustand gebracht worden ist, kowt dann ale Ausenge. Aber das Klopfen und dis ausage bratohen niont, wie in diesen Belepiel, aloh getremt zu erelgnen, sie konnen auoh glelchzel ts rorkownen. D1e ungewhnliohe Form der Ausage aer Frau in unseren Belopiel ans den Guten Lenahn agoht uns, aurgrund der klangliohen Wledertolung. eufnerkeaw in bezug auf das, was dor satz gedenklich beinhaltet. Und mit dieer etorfichen Bearbeltung sind wr aur der niloheten sture, in der Region der ferlexion.

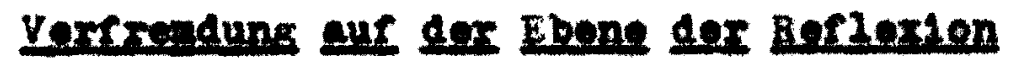
Aur der Ebene der Berlexion man der V-Efrekt mar ein

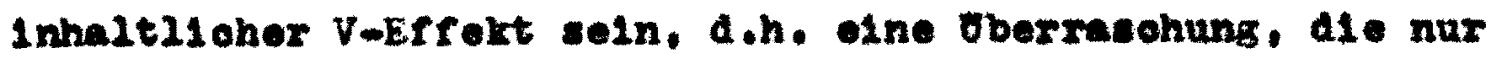
aurah elne Inhaltzohe Bedeutungertorung oder eln Bedou- 
tungs-In-Frage-Stellen zustandekommen kann. Der sinngehalt 1st auf dieser Ebene schon von vornherein in Brennpunkt.

Die Berelohe der Inhaltlichen Verfremdung sind die der Sprache und der Handlung als Mittel des V-Effekts und die der Sach- und Personenwelt als Objekte des V-Efrekts, wobel die Sprache und Handlung sich gegenseltig verfromden konnen. Dies bedeutet, daB die elnzigen inhaltilchen Verfremdungserfekte (bezogen nur auf den vorhandenen Text) die sind, die allein durch die sprache und kandlung vermlttelt werden konnen. Eine Person wird duroh lhre Sprache und ihr HandeIn verfremdet. Es konnte dann verwirrender-, aber mogI1cherwelse gesagt werden, die Person konne wiederum inr Handeln verfremden, indem sle von neuem plotzlioh gegensitzlich handele oder sprtohe, oder Indem Ihr "Charakter" Inrem Handeln widersprifohe. Doch moohte loh diese Formullerung rermelden, denn in den ersten belden Flallen ist es offensiohtlich wieder die Handlung und sprache, die das Handeln der Person verfremden, und in letzteren Pall 1st folgendes zu sagen, Der Charakter (oder Personliohkeit) einer Plgur kann sloherlich ihr Handeln verfremden, aber der Charakter der Plgur kann nux durch inr Handeln und inre sprache zum Ausdruck kommen, was wiederum bedeutet, daB die sprache und/oder die Handlung sich selbst verfremden. Wahrend die ersto Verfremdungsart auf der Ebene des Rerlexes hauptslchlioh der Begrenzung der ersten (theaterkonst1tulerenden) Illusion dient, entspricht diese zwelte 
Verfremdungsart auf der Ebene der Reflexion der Vernichtung der zwelten Illusion (eines zwingenden Ablaufs der Geschichte), wie ich in diesem Tell versuchen werde, zu zelgen.

Ich habe diesen Inhaltitchen VerfremdungsprozeB auf der Beflexiongebene in funf stufen aufgetellt, um selne Folge rom Ansatz bis zum Ende zu demonstrieren. Es ist rorausgesetzt, daB der Zuschauer bls zu der ersten Stufe dleser zwelten Vorfromdungsart sohon zur notwend1gon Aufmerksamke1t gelangt 1st.

1. Stufe. In der eraten Stufe dieses Verfrendungsprozesses wird elne gerohnliche Saohe oder elne gewohnliche Sprach-, Denk- und Handlungast in ungewdhnliohem Licht gezelgt, und 1hre bisherige Selbstverstindilohkelt wird dadurch in Frage gestellt, Eine vertraute Sache wird von einer anderen Richtung betrachtet. Sle wird sozusagen "unverstandlioh" gemaoht, um sie elgentlioh dann verstandlicher zu machen, in anderen Worteni sie wird in elnem dialektisohen Proze $B$ dargestellt.

Wenn Shul Ta dle Frage stellt, "Wle $s 011$ man slch von allen Sohwlchen frelmachen, vor allem von der todlichaten, der Lleber"81, sehen wir elne dialektisohe Zusammenstellung von zwe1 Berelohen, "Llebe" und "Schwlohe", In unangemessener Verbindung. Der Begriff "Llebe", der in der gewonnI1chen Denkhaltung zum Beroloh des Positivon gehort, w1rd

81 Eauptmann, IV, 1546. 
plotzlich als Negativum aufgefaBt. In diesem Zusammenhang wird es uns als Zusohauerm klar gemacht, daB der Begriff Llebe, der in der gewbhnlichen Denkhaltung ein Positives 1st, nicht allein im Berelch des Positiven betraohtet werden kann. Fur Shul Ta war dio Llebe durchaus negativ. D1e Polaritat dieser Berelche wird nooh welter hervorgehoben, Indem die negative selte durch eln Superlativ sestelgert wird, Die Liebe ist nicht nur elne todilche Schwlohe, sie $1 \mathrm{gt}$ die todlichste.

In dieser Stufe wird die Mogliohkelt anderer Vorstellungsmblichkelten aufgedeckt, Indem die vertraute Vorstellung als nur elne Moglichkelt von vielen gezelgt wird. Brecht sagt if vierten Nachtrag zur meorle des Mersingkaufg, "Um Gesetzlichkelten festzustellen, muB man die naturllohen Vorgange sozusagen verwundert afnehmen, das helBt, man muB 1hre 'Selbstverstindlichkelt' aurlosen, um zu lhrem Verstandins zu gelangen. Um die Gesetzlichkelt belm Fall eines geschleuderten korpers ausindig zu machen, muB man fur inn in der Phantasio noch andere MBgilohkelten sotzon; unter den gedachten Moglichkelten 1st die nattrilohe, tatstohliche dann die richtige, und die gedachten anderen Moglichkelten stellen slch als Unmoglichkelten heraus. Das Theater, das mit selnem V-Effekt elne solche staunende, errinderlsohe und krit1sche Haltung des Zuschauers bew1rkt, 1st, Indem es elne fialtung bewlrkt, die auch in den Wissenschaften elngenommen werden muB,...eln Theater des wissen- 
sohartlichen Zeltalters. Es rerwendet die Haltung, die sein Zusohauer in Leben elnnimmt, fitr das Theatererlebnis. Anders ausgedrackt, Die Elnfuhlung 1st nicht die elnzige, der Kunst zur Verfugung stehende Quelle der Gerthle." 82

2. Stufe. In der zwelten Stufe des Verfremdungsprozesses gesohioht folgendes. Duroh die Rerlexion des $\mathrm{zu}-$ sohauers und vermittelat der Evidenz in Stlok (Fakton, dis der Zuschauer bekomit) fahren aufgeze1gte w1deroproche al. Einzeltelle oder Einzelralle zur Ausprigung elnes Hauptw1derspruchs, der in selnem Entralten viele Konstellationen hat. Der Hauptwiderspruch lst gesellsohaftioher Art. Man sleht, un das vorige Belsplel weiter zu verfolgen, was elnem geschehen kann, wenn man liebt, Sobald shen Te versucht, ihre Llebe auszutben, wind sie von Sun "ausgebeutet" und inr Lebensunterhalt wird gerunrdet, aur den sie selbstrerstundich notwendig angewlesen 1st. Aber es wird auch deut11 on im Laure der Geschichte, daB Sun selbst se1nen Lebensunterhalt nicht siohern kann, ohne sohlecht zu se1n. Un 1hren ktinftigen Sohn zu lieben und inm ein sorgenloses Leben gewahren zu konnen, muB Shen Te inre Llebe zu Yang Sun aufgeben und ihn zusammen mit anderen (mittelst Shui Ta) erbermungslos ausbeuten. Herr Shu Fu 1at eln Ausbeuter, aber wenn er slch in Shen Te verliebt, wird er von Ihr (wleder als Shu1 Ta) selbst ausgenutzt usw. usw. Wenn man die zahlrelchen verschiodenen Konstellationen zusamen-

82 Hauptmann, XVI, 656. 
bringt, baut sich diese Widersprthlichke1t der Situationen zum Hauptulderspruch auf: "Man bann nlcht gut sein und auch leben", was auch abrigens als Leitmotir dieses Stuckes bezelchnet werden kann.

3. Stufe. D1e Kausal1tat dieser W1dersprtohe wird aufgeze1gt (bzw. vom Zuschauer gefunden), d.h. der widerspruch wird in selner relativen Bedingthelt als Menschenprodukt und nicht als Gegebenes gezelgt, was auch dadurch ausgedrdokt und symbolisiert wird, daB die Gotter in Guten Menschen letztiloh maohtlos sind.

Alle Zuschauer werden naturlioh nicht alle Verwendungen des V-Effekts wahrnehmen, ganz von der Verschledenhelt im Verstandnis dieser Hidersprichlichkelt zu schweigen. Es 1st auch kaum denkbar, daB Brecht elne einheltliche Wirkung auf sein Publikum mit jeden V-Effekt erwartet hatte. Wir konnen, glaube 1ch, eher das Gegentell annehmen. EIn Grand sogar fur die Vielzahl der verfremdungen in selnen sttoken w1rd zwelfellos auf das Konto der Erkenntnis dieses Aspekts der Rezeptlonsverschiedenhelt gehen. Jedenfalls wird os dem durchsohntttliohen Zusohauer (1m Idenlfall) klar, wenn nloht bel der ersten Verwendung des V-Effekts, dann vielleloht bel der hundortaten, daB das kapitalistische system, das letztlich von Menschen geschaffen murde, die Ursache dieses w1derspruchs 1 st.

Der geschlchtliche Aspokt, den Brecht far das Theater so wichtis fand, taucht in dieser Erkenntnisstufe zur 
Oberflache auf. Er betont die dialektische Lehre, daB die Gesohlohte sich in elnem stundigen VerwandlungsprozeB befindet aufgmund der "Synthosen", die aus den gegense1t1g reaglerenden W1dersprachen entstehen. "Was 1st, war nloht 1mmer und wind nioht 1mmer sein." 83 Dies bedeutet rur Brecht nichts anderes als, daB die Gesellsohart nioht lamer so geresen 1st, wie sle jetzt 1st, und daB sle in der Zukunft anders sein wird, oder, un die positive selte hervorzuheben, daB d1e in der Zukunft besser sein kbinnte.

4. Stufe. Mit der Aufdeckung der veranderbarke1t der herrschenden gesellschartlich-dkonomische Gultigkelt wird der Zusohauer dann, in der Absicht Brochts, die Synthese des dargestellten Hauptwiderspruchs selbst vollziehen. Dies entsprioht der von mir schon mehmals erwahnten "artiven" Phantagie, die durch ihre elgene Fahigke1t den "dritten Vers" mitdichtet. In Guten Menschen soll der Zuschauer d1e Losung des Problems finden. Brecht und das sttlck geben sie nicht. Der Ep1log stellt sogar direkt dem Zuschauer d1e groBe Frage: "Was könnt d1e Losung seln?...soll es ein andrer Mensch sein? Oder eine andre Welt? vielleloht nur andere Gotter? Oder kelne?"84

Nach der Schein-Losung (oder N1oht-Losung) der Gerichtsszene beginnt quas1 jetzt erst fur den Zuschauer ale elgentliche endgultige Rerlexionstatigkeit, basierend auf dem Ergebnis, welohes das sttck ze1gt, "Man kann nicht gut 84 Hauptmann, IV, 1604 . 
sein und noch leben".

5. Stufe. Der Zuschauer M1rd durch sein produktives Beteligtsein 1m Theater seine unter Umstanden neu geronnene kritioche Haltung dem gesellschaftlich-okonomischen System gegentber und sein daraus immer wleder neu entstehendes Verstandnis auf seine elgene reale gesellschaftliche Situation verwenden, von der Brecht das Abbild nehmen wollte. Daduroh, daB der Zusohauer die 1m Stllok gezelgte Entwicklung als rom Menschen gemacht erkennt und zuglelch den Grund der W1dersprtohlichkelten des im Stllok gezelgten mensohliohen Zusammenlebens und des herrschenden gesellschaftliohen systems elnsieht, wird die positive, namlich akt1ve, Selte im Zuschauer freigesetzt derart, daB dieser selne elgene gesellschartliche situation reflektlert, um selbst aktir zu werden in der Gestaltung des geselischaftI1chen systems. 85

AbschlieBend mochte $1 \mathrm{ch}$ den Zusammenhang von Verfremdung und Illusion betrachten, um die jewelligen Entsprechungen belder Komplexe auf inre Struktur hin durchsichtig zu machen.

Es hat sich durch diese Untersuchung ergeben, daB

85 Genau an diesem Punkt hat man dann oft rersucht. die politisch-marxistisehe Konzeption in Brechts stioken realisiert zu finden in dor Welse, daB man zu sehen glaubte, daB Breoht das kapitalistische system in negativer Form darstellte mit der Wirkungsintention, dem Zusohauer die negativen Auswirkungen des Kapltalismus zu zelgen, um $1 \mathrm{hn}$ auf die marxistisch-sozial1 stisch ausgeprigte Gesellsohaftsform hinzurthren, die oben - so gesehen - elne viel bessere, und d.h. menschonwdrdigere Gesellschaft anbleten wurde. 
elnerselts die theaterkonstitulerende Illusion und die Verfremdung auf der Ebene des Reflexes in Opposition zueinander stehen (s. Selte 47), und daB andererselts die Illusion einea zwingenden Verlaufs der Geschlchte und dle Verfremdung auf der Ebene der Beflexion elnander entgegengesetzt sind. Diese Entgegensetzungen sind $\mathrm{es}$, durch welche sie sich aufelnander bezlehen.

D1e Verfremdungen auf der Ebene des Reflexes mechen die Illusion, man habe es mit der Realitat $z u$ tun, durch Ihre den Zusohaver storende W1rkung immer wleder punktuell zuniohte, wodurch, auf das Ganze gesehen, dlese Illusion begrenzt bzw. klein gohalten w1rd. Die verfremdungen auf der Ebene der Reflexion bezlehen sich aur die Illusion elnes zwingenden Verlauf der Geschlohte Insofern, als diese die W1derapriche im Geschehen aufzelgen zusamen mit der Beflexion auf die Moglichkelt der Macht und des Eingreifens des Menschen, wodurch die Kausalitat der Geschehnisse aurgedeckt wird, so daB dlese IIlusion sioh aurheben muBi das hat zur Xonsequenz, daB dlese Art der Illusion auf diese We1se rbII1g ausgesohaltet werden soll, we es berelts rorher schon gesagt murde.

Auf diese zentrale Funktion des 2. Tomplexes hin gesehen kann der VerfremdungsprozeB wle folgt zusamengefaBt worden. Die 1. Stufe dlent der Hinfunrung zu den Wldersprachen, die 2. Sture erbringt die Tatsache der Widerspriohe, d1e 3. Stufe ze1gt den Grund d1eser Widersprtiohe, die 4, und 
5. Stufe bestimmen sich als die Folgerungen aus dieser "Elnsloht" und bezlohen sloh direkt auf den zusohauer, nimlioh die 4. Stufe belnhaltet die Porderung der Antwort auf die Frage, durch was elne Veranderung zu gestalten se1, die 5 . Stufe betrifft die Forderung nach der Anwendung der Erkenntnisse auf die elgene gesellschaftiche situation.

Zur Volletundigkelt dieser eben skizzlerten Struktur se1 noch kurz daran erinnert (s. Se1te 27f.), dab neben der Prennung der belden Illusionsarten auch elne gegenseltige Beelnflussung gegeben 1st. Dementsprechend kann die Trennung der belden Verf rendungsarten auch nloht so scharf gezogen werden, wie es oben dargestellt worden 1st, well sich belde wiederum aufelnander beziehen. Dies soll jetzt erlutert werden.

Zur Illusion, Auf der elnen Selte wird durch die vernichtung der Illusion elnes zwingenden Verlaufs der Gesohichte (2. Illusion) zugleich eine punktuelle Aurhebung der theaterkonstituierenden Illusion (1. Illusion) berirkt, aur der anderen Selte hilft die punktuelle Vernichtung der 1. Illusion an der Vernichtung der 2. Illusion mit.

Zur Verfremdung, Elnerselts fuhrt die Verfremdung auf der Ebene des heflexes (1. Verfremdung) zu der Verfremdung auf der Ebene der Reflexion (2. Verfremdung) hini andererselts bezleht sich die 2. Verfremdung 1ndirekt auf die erste Verfremdung, und zwar so, daB die 2 . Verf remdung zunllohst die 2. Illusion aufhebt, durch welche (wie oben ge- 
sagt) die 1. Illusion aufgehoben wird, was zur Folge hat, daB die 2. Verfremdung eben vermittelst 1hrer Ausschaltung der 1. Illusion auch die 1. Verfremdung in gewissem Sinne beinhaltet.

Um elnen kurzen Vorblick auf den welteren Verlauf zu geben, Im nlichsten Tell geht es darum, das Hauptmittel des Brechtschen V-Effekts, nlmlich die Sprache, zu analysieren. Melne Z1elsetzung 18t es. Breahts Methodik in seiner Sprachgestaltung hinsichtlich selner gesellschaftliohen Intention zu demonstrieren. Dabel wird diese Untersuohung ouf das Wesentlichste, und d.h. auf die Inhaltliche Selte der Sprache. beschrankt, was zur Polge hat, daB d1e Verfremdungen auf der Ebene des Beflexes nioht behendelt verden. Auoh wird in den angerahrten Belspielen der Verf rendungsprozeB auf der Ebene der Reflexion nioht augefunrt, sondern es solien zugunsten des Wlohtigsten, d.1. Ale Intention Brechts, nur Hinwe1se auf $1 \mathrm{hn}$ gegeben werden. 
UNTERSUCHUNG DES HAUPTMITTELS DER VERFREMDUNG

\section{EINLEITUNG}

In dieser Untersuchung hat sloh chon herausgestellt, daB es Im geschriebenen Text zwel Hauptquelien des Brechtschen Verfremdungseffekts glbt, namlioh die sprache und die Handlung. D1e Sprache steht unmittelbar vor den Augen des Lesers und hat den Vorte11. Objekt einer alustisohen und optigchen Examination zu sein, whrend die Handlungen me1st 1nd1rekt (und daher weniger konkret) aus der sprache cozusagen herauggelesen werden mtlsan.

Da $10 \mathrm{~h}$ in dieser Arbelt nur die sprache auf ihre Verfremdungen hin untersuohen rerde, so mbchte 1 oh an dieser stelle dieses Vorgehen zu rechtrertigen suchen.

Die Handlung we1st elnen veniger direkten und konkreten Charakter als die sprache auf, da der sprache ein bestimmtes System von Zelohen (beruhend auf der Obereinstimmung der Glieder einer bestimten sprachgeneinschaft) zugrundellegt, deshalb 1st es moglioh. sion bel der Untersuchung der sprache auf elne Norm zu bezlehen, die wlederum die MOglichke1t anbietet, in wisenschaftlicher Hinsicht diese im Stuck enthaltenen Konkretionen der Sprache zu betrachten. Die Handlung hingegen, der Brecht ebenfalls ein groBes Interesse gewidmet hat, beinhaltet nioht in dem fur 
die Sprache geltend gemachten Sinne dieses aberliche Gerist, das als wissenschaftilches objekt dienen könnte. Der letzte Grund besteht darin, daB alles in der Betrachtung des geschriebenen Textes, letztlich auch die Handlung, durch die sprache vermittelt wird. Das bedeutet, daB da, wo die Handlung sich abert, auch de Sprache betelligt 1st (abgesehen von den wenlgen Bthnenanwelsungen, die die Handlung auch vermitteln). Daraus ergibt sich, daB die Sprache das Hauptmittel der Verfremdung 1st.

D1e Notwendigkelt elner naheren Analyse dieses sprachI1chen Mittels, um Brechts Verfremdungstheorle genaustens zu verstehen, sthrt uns jetzt zu elner detallilerteren Untersuchung Brechtscher Texte, und zwar bis zu elner Betrachtung subtilerer sprachlicher Nuancen. Ich halte mich, wo mogl1ch, an den ausgewahlten Guten Menschen-Text, dooh kommt es gelegentlich vor, daB ein deutlicheres Belspiel aus einem anderen Brecht-stick mitelnbezogen w1rd, dann aber nur, um einen bestimmten Punkt bezreiflioh zu machen, wo Dex gute Mensch sich als nicht ausrelohend erwelst.

\section{DIE SPRACHE}

Elne Frage, die man sioh hinsiohtlich der sprachl1ohen Untersuchung stellt, wenn man da eine Verfremdung finden mochte, bezleht sioh auf die allgemelne Mogliohkelt dieses Verfremdens. Wieso kann man uberhaupt die Sprache verfremden? Was fur Momente liegen in der Spraohe vor, die da- 
zu geeignet sind? Wie loh erortert habe (s. Selte 37fr.). entzieht das Verfremden dem Betrachter die Selbstrerstandl1ohke1t des betrachteten Objekts. Wenn man diesen Punkt aberlegt, l1egt der Gedanke nahe, daB d1e vorausgesetzte Basis fur ein verfrendendes verfahren gerade in der selbstverstundlichicelt liegt. Das selbatverstundliche in der Sprache hat aber auch seine Ursachen, die sich in der Festigke1t befinden. Und diese Festigkelt mochte 1 ch jetzt in den Blick rtcken, denn darin sind letztlich die Sohldssel zur Brechtschen Methode der sprachlichen Verfremdung zu sehen.

D1e Selbstrerstandliohke1t in der sprache beruht auf einer Festigke1t, die sich in zwel slch gegenseltig beelnflussende Berelche tellt, Festigke1t der Denkwelse und der Sprache. Ich will mich nlcht lange bel der Erorterung des Verhaltnisses zw1schen Denken und Sprache aurhalten, da diese weit tber den Unfang dieser Untersuohung hinausginge. Es gentlgt, glaube $1 \mathrm{ch}$, darauf hinzuweisen, daB das enge Verbundengein von Denken und Sprache elne bekannte Festatellung 1st, und daB dieses Verhaltnis sich nicht in der Tatsache erschopft, daB die Sprache lediglich als Kommunikationsmittel zur Mittellung der Gedanken dient, sondern daB die Sprache auch beim Denken selbst elne Rolle splelt. 86 Es

86 Gert Jager in D1e deutsche Sprache, Hrsg. Erhard Agricola, Wolfgang Fleischer und Helmut Protze (Le1pzig. VEB B1bliograph1sches Inst1tut, 1969), S. 31ff. Von jetzt an als DdS angefthrt. 
1st noch nicht wissenschaftlich gezelgt worden, inwlewelt die schon bestehende Sprache das begriffliche Denken formlert, aber das Vorhandenseln dieses Elnflusses laBt sich nicht abstrelten. Auf diese Tatsache mich statzend mochte $1 \mathrm{ch}$ behaupten, daB dle sprachllche Verfremdung, wle Brecht sle verwendet (abgesehen von den schon erwahnten sprachl1chen Verfremdungen, die auf der Ebene des Reflexes statt(inden ${ }^{87}$ ), sich telis auf die Festigkelt der sprachlichen Formulierung konzentrlert und tells auf die Festigkelt der Denkweise, die nicht immer elne festgepragte sprachliche Formulierung benutigt, sondern in elnem fixen Rahmen eine gewlsse Freihe1t im Verkntipfen der Worter erlaubt.

\section{Eestigkelt der sprechllahen Formulierung}

Die Festigkelt der sprachlichen Formullerung, und das helBt Wortverbindung, wird durch drel Momente bestimnt. namlich 1) wie haufig die Formulierung gebraucht wird, 2) wle groB die Auswahl unter den Verknlpfungspartnern 1st und 3) wie stark die einheltilche Gesamtbodeutung von den Bedeutungen der Bestandtelle abweloht. 88 Das erste Moment bedarf kelner langen Erlduterung. Es 1st einleuchtend, daB eine Wortverbindung sich dem SprachbewuBtsein um so fester einpragt, je hulfiger sie verwendet wird. Das zwelte Moment bezlent sich auf die syntaktische und semantische Kongruenz,

87 Wechsel der st1lebene (slehe s. 56ff.), Xlang der sprache (siehe S. 59), und Fragen (siehe S. 55f.). 88 Erhard Agricola in DdS, S. 586. 
die belde eine beschrankende W1rkung auf die Auswahl der Verbindungapartner haben. D1e Moglichkelten elner syntakt1 schen Wortverbindung sind durch die grammatischen Bedingungen bestimmt. 89 ober die Auswahlbeschrknkungen grammatischer Art hinausgehend werden die Wortverbindungen in Hinblick auf inre Bedeutung elngeschrankt. Diese sogenannten Bedeutungsbeziehungen wurden zuerst von Walter Porzig 90 beschrieben und dann (unabhang1g und orfenbar sich der Existenz des Porzigschen Aufsatzes nioht bemat) von Ernst Leisi" als "semantische Kongruenz" welter entwiokelt. Es handelt sich um die Bezlehung zwisohen Verb und subjext, Verb und objekt, Adjektiv und substantiv usw. Zum Belspiel, Das Verb "bellen" tritt nur zusammen mit dem Subjekt "Bund" auf, und diese Wortverbindung bildet ein einfaches kleines Feld (1rrefuhrenderwelse "syntakt1sches" Feld genannt ${ }^{92}$ ), das nur durch obertragung vergrobert werden kann (bellenKanone). Aber trotz der tbertragung blelbt dieses Feld relativ klein. Schon das Verb "springen" bringt elne grobere Subjekt-Auswahl mit sich. Eln Belspiel eines kleinen VerbObjekt-Feldes 1st "rallen-Baum" (gelegentlioh auch "Recke"

89 Wilhelm Bondz1o in Das. S. 555.

90 Walter Porz1g, "Wesenhafte Bedeutungsbezlehungen," PBB, $58(1934), 70-97$.

91 Erngt, felgi, Der wortinhalt (Heidelbers' Quelle und Meyer. 1967), $5.68-70$.

92 ple Bezelchnung kommt von Walter Porzlf wunder der Sprache (Born! Franoke Verlag 195?): S. if ftr GoIn vetonen, daB es Wortfelder gibt, die erst im Satz wirkam sind: im Gogensatz zu den "pérataktischen Foldern", bel denen nur Substantive oder nur Verben oder nur Adrerb1: on oder nur Prapositionen usw. nebeneinandersesteilt werden. 
und "Urte11"). Das Feld "blond-Haar" steht ftir elne Adjekt1v-Substant1v-Beziehung. Andere Feldbeziehungen lassen s1ch durch das AussohlieBen bestimmter worter lelohter erklaren, so kann man die Rlchtungaadverbien "her, hin" nicht mit Verben verbinden, die elnen Buhezustand ausdrucken, w1e z.B. "gchlafen", "ruhen", "liegen" (Verb-Adverb), und "zart$11 \mathrm{ch}$ " kann zwar be1 "rlustern" oder "atreloheln" ersche1nen, doch nlemals bel "fahren", "esoen", oder gar "schreion" und "schimpion" (Adverb-Verb).93 Das dritte featigkeitsbestimmende Moment, nimlich die Abrelchung der Gesemtbedeutung elner Wortverbindung von den Bedeutungen der Bestandte1le, bezieht sich auf die Idiomatisierung, das helBt, aur die Verbindung bestehender worter in der Welse, daB sich ihre Bedeutungen bel bestehenblelbender Trennung der Formen zu einer neuen, besonderen Gesamtbedeutung vereinigen. $94 \mathrm{Je}$ starker die Abwelchung dieser Gesamtbedeutung von den einzelnen Bedeutungen der Glieder 1st, desto fester 1 st die Wortverbindung.

Die Wortverbindungen, die duroh die drel oben genannten Momente elne gerisse Festigkelt errelchen, nennt man

\section{Bondz10. 3. 555.}

94 Agr1001a. S. 586. AuBer der Id1omat1 s1emung g 1 bt es naoh Agr1001a zwe1 andere Moglichkeiten fur die Entatehung von Redewendungen $(S, 587)$. elnmal durch den Ebertritt von festen Einheiten aus dem Bereloh der Sprichworter, Sinnspriche, zitate uar. in die Kategorle der Rederendungen, zum anderen durch die wortliche oder frele obersetzung von Redewendungen aus elner Fremdsprache. 
Rederendungen.95 Die Grenze zwisohen rest und nicht rest 1st schwer festlegbar. D1e Problemat1k diesbeztglioh wird von Erhard Agr1cola96 durch das folgende Belapiel elner "Iosen" Wortverbindung 11lustriert, D1e lose Verbindung "saure Gurke" bezelchne zwar melst die nach elnem beatimmten Rezept zubereltete Gurke, doch sel es mogl1ch, dab man elne Gurke meine, die sauer sel im Verheltnis zu wenlger sauren Gurken. Elne Umstellung dieser Wortverbindung sel sogar erlaubt ("dle Gurke 1st sauer" oder "sehr saure Gurke"). Ohne daB diese Modifizlerungen als storend emprund en wirden. Diese Freihelt habe man aber nicht mehr bel elner Verblndung w1e "der velBe Tod" deron grammat1sche Struktur zvar dieglelche sel, aber duroh die spezielle Variante von "we1B" bedeutungsmaB1g zu der unveranderbaren Gesamthelt "Tod in Schnee und E1s" verschmolzen se1.

Der Gesamtsinn soloher Wortverbindungen wle "der we1Be Tod" oder "blinder Alarm" In denen sich schon die Bedeutung des elnen Gliedes (we1B, blind) sehr weit ron se1nex Hauptbedeutung entfernt hat, 1st noch aus den einzelnen Gliedern zu erschl1eBen. Und obrohl die Bedeutung des einen Gliedes austauschbare Varianten hat ("der duroh E1s und Schnee verursachte Tod". "1rrttml1oher/unnbt1ger Alarm"), alnd solche Vortverbindungen fest genug, um Abrandlungen in der Zusammenstellung wie "sein Tod war we1B" oder "eln sehr

95 Agricola, S. 586.

96 Ebenda. S. $586 \mathrm{ff}$. 
blinder Alarm" zu verbleten. Sle bilden den obergang zu den "elnfachen Bedewendungen".

Be1 den elnfachen federendungen (z.B. "Abschled nehmen". "Anordnung treffen", "In Erwlgung ziehen") 1st ein Gl1ed, melstens das Verb, abgeblaBt oder zum Tell ungedeutet. Der Gesamtsinn 1st aber nooh aus den Einzeltelien zu entschltsseln, und die worter konnen noch in sehr begrenztem MaBe gegen bedeutungsnahe ausgetauscht werden.

"Feste Rodewendungen" dagegen ( $\mathrm{z}, \mathrm{B}$, "OI 1ns Feuer gleBen", "mir geht ein Licht auf") haben elne relatir feste inhaltilohe Bindung, die nur vereinzelt duroh bedeutungsuhnl1che worter ersetzt ("auf der Palme seln/sitzen") oder andere Wbrter erweitert werden kann. Die Bezlehung der E1nzelglieder 1st zwar durchsioht1g, d.h. die wortliohe Bedeutung hat schon elnen SInn, aber die Gesamtbedeutung kann dennoch nicht unmittelber exschlossen werden. S1e muB eine verallgemelnerte oder tbertragene sein, deren Schlussel melat die blldilch-metaphorische Form elnes inrer Glieder 1st. Obwohl manche Redewendungen elgentlich und metaphorisch zu verwenden sind ("tber den Berg sein"), gilt melst nur die abertragene Form.

Der Gesamtsinn von "erstarrten Redewendungen" ( $z \cdot B$. "Jemanden im St1ch lassen", "etwas aufs Tapet bringen", "das geht mir aber die Hutschnur 1") ist dermaBen umgedeutet, daB er vom gegenwartigen Sprachgebrauch aus gesehen unbegrtindet 1st. Der Sinn der Verbindung 1st auch nioht aus den Be- 
standtellen abzuleiten. Diese Rederendungen sind unzerlegbar, unablthzber und konnen nur ausnahmswelse erganzt werden. Das ubertragende B1ld 1 st melstens durch die historische Entwicklung so verblaBt, daB die Qbertragene Bedeutung, far die das B1Id stehen soll, das Elnzige 1st, was w1r heute noch haben.

"Festgepragte Satze" haben auBer der tber die Redewendungen hinausgehenden Struktur (d.h, sie sind in sich geschlossene Aussagen) alle Merkmale der Redewendungen, sind aber noch begrenzter in der Abwandelbarke1t. Belsplele dioser Kategorle sind, "hast du Tonel", (ooh) du ahnst es nlohtI", "das haut elnen hin (um) 1", "loh denke, mloh laust der Affel", "das (also) 1st des Pudels Kern!" Diese satze, d1e zum Te1l tatslohlich zitate und derglelohen sind, b1lden den vbergang zum Berelch der spriohworter, Z1tate, gefldgelten Worte, Sentenzen, Sinnsprtiche und Aphorlsmen. Fur unsere Zwecke brauche $1 \mathrm{ch}$ an dieser stelle nicht welter In eine Beschrelbung der Form und des Wesens dieser Wortverbindungen hinelnzugehen als zu sagen, daB sle melst elne erstarrte Festigke1t bes1tzen, die aufgrand des wiederholenden, oft lehrhaften Charakters Abwandlungen und wortersetzungen selten gestattet.

Es darfte gefragt werden, we dlese Aufgllederung der Wortverbindungen mit Brecht zu verelnbaren 1st, W1e $1 \mathrm{ch}$ am Anfang dieses Telles der Untersuchung angedeutet habe, ist es melne Absicht, zunlchst die Frage nach der allgemelnen 
Verfremdbarke1t der sprachlichen Formulierung zu behandeln. Ausschlaggebend ist die Festlgke1t dleser Formulierungen, da die Hafigke1t der Erschelnung von festgepragten sprachlichen Formeln sle in die Selbstrerstandliohkelt des Sprachbemabtseins einbettet, in der Regel sind die Formulierungen um so selbstrerstandlicher, je rester. Aber so schlecht diese Situation von Brechts Standpunkt aus gesehen seln mag, 1st sle nicht aussichtslos. Gerade well die Festigkelt die Selbstrerstandlichkelt konstatiert, bringt elne stelgerung In der Festigkelt der sprachlichen Formulierung elne ste1gerung in der Verfremdbarke1t dieser Formullerung mit sich. Das helBt, EIne elnfache Verthderung einer "Iosen" Wortverblndung zum Belsplel, die kelne wirkliche Festigkelt aurwe1st, stort das SprachberuBtsein des Horers/Lesers kaum oder gar nloht. Aber die Verlinderung elner Wortverbindung, die festgeprlgt 1 st und harig gebraucht w1 rd, raubt dem Leser oder Borer die bequeme Selbstverstundlichkelt der urspranglichen Formulierung ("das 1st des Sohwelnes Kernl"). Der Grund, warum Brecht ein so groBes Interesse an der sprachl1chen Verfremdung hat, 1st, wle immer, gesellschartlich bestimmt. Gerade die Selbstverstandichkelt will Brecht storen, denn "selbstverstandiloh finden" he1Bt so viel wie "nlcht mehr reflektierend beobachten und kritisch bearbelten" - eln Zustand, den er fur besonders gerahrlich halt, da "selbstrerstandlich" langst nicht "wahr" bedeutet, und weil Selbstverstandlichkelten in der Sprache Selbstrex- 
standichkeiten im Denken vertreten, bedingen, ja verfest1gen sogar. Hinsichtlioh des gesellschaftliohen Denkens fordert es den Marxisten Brecht geradezu dazu heraus, diesen veranderungsfelndlichen Zustand zu bekampren. (Auf diese Intention Brechts bin $1 \mathrm{ch}$ an mehreren Stellen schon elngegangen.) In welcher Welse dieses Beklmpfen vor sich goht, wird jetzt ausufthren sein.

\section{Verfremdung selbstverstandlich gewordsner Formullemungen}

Brecht verfremdet solohe Formulierungen, die uns durch Ihre Festigkeit selbstrerstandilch geworden sind, durch vier Hauptmethoden, 1) durch direkte Vertunderungen, 2) durch unangemessene Verwendungen, 3) durch relativierende Nachsatze und 4) durch wortwortiliche Auffassungen.

D1rekte Verlnderungen. Die erste Methode grelft die Festigkelt der Wortorinung und Wortverbindung direkt an, das AuBere der Formulierung sozusagen. Far Verfremdungen dieser Art erwelst sich Mutter Courage und Ihre Kinder als sehr fruchtbar. An einer Stelle, wo Mutter Courage angeslchts elnes unvorausgesehenen Uberfalls der Katholiken auf die Evangelisten, bel denen sie sich aufhalt, das Gesicht inrer Tochter Kattrin mit Asche einreibt, um sie vor den Gewalttaten der Soldaten zu bewahren, rechtfertigt sie dies mit der Aussage, "Sein Lloht muB man unter den Scherfel stellen, he1Bt es."97 Zunkohst wird das Bibelzitat 
dadurch verfremdet, daB seine auBerliche ordnung gestort w1rd, und weiterhin, weil es in ungekehrten sinne von Mutter courage wledergegeben wird. Die auf Verallgemelnerung beruhenden Autoritaten, wie in diesem Fall die B1bel, werden duroh solche Verdrehungen automatisch in Frage gestellt, und durch die entgegengesetzte 1nhaltliche 0rdnung des Z1tats w1rd man auf die verdrehte ordnung des Lebens in e1ner Gesellschaft, die rom Krieg orhebllch mitgenommen wird, aufmerksam gemacht.

In Guten Menschen reraucht Wang fur die drel Gotter Obdach zu finden. Als er elnen Hausbesitzer fragt, ob er die Gotter aufnehmen konnte, schreit der Hausbesitzers "Versohone uns mit delnen Gottern! W1r haben andere Sorgen 99 Hier wird olne relativ feste pormulierung ("Verschone uns mit delnen Klagen (") durch ein Ersetzen des letzten Wortes, das aufgrund der Natur der Aussage 1mmer in den Berelch des Negativen hinelngedrangt w1rd, durch ein Wort, dessen Inhalt im normalen Sprachgebrauch elnen hoheren, erhabeneren Wert hat, verfremdet, DaB die Gotter plotzlich ihre selbstrerstandilche, respektierte Position der Allmachtigen verlieren und von den Mensohen als nicht mehr als nur "Sor-

In der Sprachgesteltung Bertolt Breohts," Diss. Bonn 1960, S. 35. Das 21 tat 1st bel Hauptmann, IV, 1377 zu rinden. Be1 Hatth. 5, 15 helBt es, "Auch zundet man kein Iloht an und stellt os unter den Scheffel, sondern auf den Leuohter."

98 Heuptmann. IV. 1491. 
gen" behandelt werden, fthrt darauf hin, daB Breoht die Idee der gottgegebenen Zustandlichkelten nicht1g machen w111. Neoh Breoht 1 st des gesellsohaftliah-8konomleche System ron Menschen gemacht, nicht ron Gottern. Und die Menschen haben also auch allein die Macht, dieses System wlederum $\mathrm{zu}$ verandern.

E1nen thnlichen Fall finden wir euch in Mutter Courage. Als Mutter Courage und der Feldprediger spekul1oren, ob der Krieg bald zu Ende gehen kbnnte, well Mutter Courage aberlegt, ob sle neuen Vorrat elnkaufen soll, sagt der Feldprediger, es kbnne zu elner klelnen Pause kommen. Plotzlich könne der Krleg "an was Unvorhergesehenem Ins Stocken kommen", viellelcht eln obersehen, und "das sohlamassel" sel da. "Und dann kann man den Krieg wleder aus dem Dreck ziehen "99 "Etwas (melstens den Karren) aus dem Dreok ziehen" bedeutet "etwas in Ordnung bringen" Verfremdend wirkt die Verwendung des Wortes "Krieg" anstelle von etwas, was zumindest in Ordnung seln kann. D1e Haltung des Feldpredigers, die or mit elner lacherlichen Selbstrorstund110hke1t trät, drtokt aus, daB der Krieg etwas wirdiges und Gutes sel, diese Haltung soll nach Breoht wieder das vol11g verkehrte Gesellsohartsaystem (den Kap1tal1smus) aurzelgen, in dem der Krieg ein Element der Eristenz 1st. 100

\section{Hauptmann. IV, 1402.}

100 Ebenda, $X X, 271$. Breoht Z1tat, "Volker, die innerlich so aufgebaut sind wie die unsem, nimlich kapitalistisch, brauchen tatskchlioh Kriege, um existieren zu könen." 
Elne weltere verf remdende veranderung elner mehr oder wenlger featen Wortverbindung bezieht $81 \mathrm{ch}$ auf elne andere auoh in Mutter Courage $81 \mathrm{ch}$ befindende Ansplelung auf die hohe Elnschatzung des Krieges. Die Courage kauft aufgrund des Rates des Feldpredigers neue Vorrate ein. Aber in selben Jahr "droht" der Frieden 1hr Geschaft zu rulnieren, da im Frleden sie ihre Waren nioht verkauf on kann. Auf die verbrelteten Frledensgerdachte hin sagt Mutter Couragel "Sagen Sle mir nicht, daB Friede asgebrochen 1at, wo $1 \mathrm{ch}$ ben neue vorrate eingekauft hab." 101 Hier wird elne harig verwendete Hedewendung "der Krieg 1 st ausgebrochen" dadurch verfremdet, daB das Wort "Kr1eg", das elnen durchaus negativen Sinngehalt hat, mit selnem di rekten Gegensatz ausgetauscht w1rd. Durch d1eses Ersetzen erhalt das wort "Frieden" elne stutz1gmaohende pejorative Bedeutung und stellt dadurch die Frage, ob die selbstrerstundliche Denkwelse, die Frieden als Positivum im Kapitalistischen System (In diesem Sthok mit dem Fasch1smus verbunden) setzt, tatskoh11ch fur selbstverstandilch gehalten werden kann (slehe das rorige Beispiel). Angesiohts der Haltung von Mutter Courage scheint ein solcher Gedanie nicht nur fehl am Flatz zu sein, sondern direkt ralsch. Denn hier unterstatzen sioh belde Elemente (Krleg und Kapltallsmus) gegenseltig. Unangemessene Verwendungen. Verf remdungen von selbatverstandlich gewordenen Formullerungen mbsen, wie gesagt, 
nicht direkt verundert werden. Sle konnen also in ihrer resten gewohnlichen form stehenblelben und trotzdem in einem neuen Licht gezelgt werden oder selbst einen anderen Inhalt verf remden. Unangemessene Verwondungen, das he1Bt, feste Formulierungen, die sich in Kontexten befinden, zu denen sie elgentilch nicht passen, greifen nicht die Festigkelt der AuBerliohen Struktur der Formulierung an, sondern die Festigkelt der Gebrauohswelse der Formulierung. Eln entsprechendes Beispiel kommt an der stelle im Guten yengehen ror, wo Prau Yang erzlhlt, Ihr Sohn Sun habe sloh aus elnem "verkommenen Menschen in olnen natzlichen" rerwandelt. Sie hat Yang Sun zum "Tabakkon1g" Shul Ta hingebracht, der inm "ehrliche Arbelt" rtr selne Rehabilitierung anbletet. Die dankbare Prau Yang sagt dann zu Ihrem Sohn mit der Hoffnung. er wirde slch durch die Aufnahme in die Tabakfabrik bessern. "Du b1st vom rechten Weg abgew1chen. Versuch nun, durch ehrliche Arbelt wleder so welt zu kommen, daB du delner hutter in die Augen sohauen kannst." 102 In diesem Kontext nimmt die biblische Bedewendung "rom reohten Weg abrelohen" e1ne kap1tal1smusunterstatzende Bedeutung an. Nech Frau Yang 1st "der rechte Weg" der heg zum Aufatieg in der kapital1st1sohen Gesellschart. Der uraprangliche blblische Gebrauch dieser Formulierung, die eine Abkehr von der ohrist11 chen Eth1k besagt, wird 1nsofern verfremdet, als die Redewendung hler ausdrticklich die Abkehr ron der Lebenswelse 
auch des erfolgrelchen Kapitaliston beze1chnet. DaB 1m Kapital1smus der Mensch "nttzl1ch" seln muB, 1st elne typ1sche Brechtsche Andeutung. In der Kap1tal1stischen H1erarchle, in der Sun selnen Aufstieg zum "besseren" Menschen vollziehen soll und dies im Sttok tatslohlich errelcht, setzt dio Existenz "natzlicher" (und das he1Bt fur Frau Yans "guter") Menschen dies voraus, dab diese Menschen sich erst dann als "nutzlich" erwelsen, wenn sie durch kapitalistische Mittel benutzt, oder besser gesagt, ausgenutzt werden, und weiterhin, daB, un augenutzt werden zu konnen, d1e Anwesenhe1t von Ausnutzern vorausgesetzt 1st. Duroh die unangemessene Verbindung dieser Formulierung mit dem Kap1tal1smus wird die entsprechende Denkwelse, die kelneswegs nur fur Frau Yang ei ne Selbstrerstandlichkelt 1st, macht18 in Zweifel gezogen.

E1n wenlger direkt wirtschaftsbezogenes Be1splel e1ner Verfremdung durch eine unangemessene Bezlehung der Formulierung zum Kontext l1efert uns Wang im Guten Kenschen. In seinen Bemuhungen, ein zimmer fttr die Gotter zu finden, wid er von den vorbelgehenden Herren abgelehnt. In seiner daraus resultierenden Wut ruft Wang ihnen nach, "Die Gotter schelBen auf euch!"103 Die gebrluchliche derb-vulgare Redewendung "auf etwas/jemanden scheiben" bedeutet im metaphorischen Sinne "auf etwas/Jemanden verzichten". Zwar wird die Redewendung in ihrer Form und auch in 1hrer Bedeutung

103 Hauptmann, IV, 1494. 
nicht angetastet, und dies he1Bt, daB elne Verfremdung der Formullemung als solche nloht vorliegt, jedooh kommt eine Verfremdung daduroh zustande, daB diese besondere Redewendung, deren naturalistischen Ansplelungen in vornehmeren Zusammenhangen zumindest hochstungewohnlioh 1st, gerade mit elnem erhabenen 1dealialerten Sinngehalt verknuprt wird. Diese Verfremung der Gotter dient wleder dazu, die Gotter als nicht erhaben, nicht allmalchtig, 1m Gegentell, als nichtig zu entlarven. Hier wird noch einmal durohsich118, was Brecht letztlich damit sagen will, nimlich, Die Gotter haben die Welt, wle sle 1st, nlcht gemacht, sondern d1e Mensohen, die auch dazu befahigt sind, s1e zu verdndern. In den Brecht-Werken kommen oft Belspiele dieser Kategorle ror, besonders in Verbindung mit der Beligion. EIn anderes Mal sagt Wang zu den an der H1lfsbereltschaft der Stadt zwe1felnden Gottern, um sie rom Gegentell zu tberzeugen, "Alle Finger leokt man $81 \mathrm{ch}$ danach, euch zu bewirten."104 D1e Gegenuberstellung von Erhabenem und N1edr1gem verfremdet zundchst, aber hlor 1 st os bestimmt nioht ein Zurall, daB Breoht elne Redewendung gebraucht, die s1ch aus dem Essensberelch herle1tet. D1ese Verknapfung hebt die Tatsache herror, daB es in dem kapitalistischen Gesellsohaf tssystem Hunger gibt (w1e in Sezuan), obwohl es oft elne Selbstrerstandlichkelt in der Denkwe1se dieser Gesellschaft 1st, dab die Macht einer Veranderung der Zustand-

104 Hauptmann, IV, 1491. 
lichke1ten in der Hand Gottes liegt. Es rullt in diesem Sttlok auf, daB nur arme Menschen daran glauben, whrend die Relohen entreder sloh den Gottern gegenuber 1mmer ablehnend verhalten, wie bel Wangs Versuchen, oder sie werden gar nloht von Brecht mit den Gottern in 1 hrer gottlichen Gestalt in Verbindung gebraoht, sondern nur in mensohlicher Gestalt. Dies soheint mir eln Zelchen darar zu sein, daB Brecht sagen will, die Menschen mit Geld sind sich sohon Ihrer elgenen Macht bemuBt und wissen, daB Ihre Macht duroh das Okonomische system geschaff on wird, whrend die Armen sloh Immer noch auf die leere Hoffnung elnes gottlichen EIngrelfens stutzen. DaB die Gotter keine gottliche Macht haben. wird an zahllosen Stellen dargestellt. Die elnzige reale Macht, die von den Gottern in diesem Stack augedbt w1rd, manifestiert sioh elnzig und allein in der Form des Geldes, das sie Shen Te geben. Sonst erhalten sie nur duroh das Glauben der Menschen (Shen Te und Wang) und als Richter verkle1det (also Menschen) elne schelnbare tberlegenhe1t. D1ese Idee unterstutzend 1st es eine Tatsache, daB die elnzigen Menschen 1m Sttlck, die an die Gotter glauben (Shen Te und Wang), dlejenigen sind, die melsten unter dem system zu lelden haben. Dam1t ist ber noch nloht geangt rorden, daB Brecht die christliche Eth1k ablehnt. Eher 1 st das Gegentell der Fall, denn die Idee der Nuchstenliebe scheint zumindest ron diesem stack aus gesehen kelne unsympathische gesellschaftilche Haltung zu sein, nur zelgt sie sich in 
diesem System als selbstrernichtend. Wogegen Brecht angehen w111, 1st der Glaube der Armen, daB sie aus ihrer s1tuation durch etwas wie einen Deus ex machina gerettet werden ganz das Entgegengesetzte wird im Guten Menschen Gargestellt. Die Gotter missen sich selbst auf einer "rose Wolke" aus $1 \mathrm{hrem}$ untberwindbaren D1lemma herausheben. Eln anderes Belspiel elner Verfremdung durch die unangemessene Verwendung elner festgepragten Formulierung befindet sich in Mutter Courage. E1115, der alteste Sohn der Anna Flerling, versucht mit selnen Soldaten Ochsen von den Bauern fur das Heer zu stehlen. Er wird von den Bauern gefangen, aber duroh seine Sohlauhe1t 1st er 1mstande, die Bauern umzubringen und zwanzig Binder mitzunehmen. Der Feldhauptmann lobt inn und rechtfertigt die Tat daduroh, daB E1I1f etwas Gutes far des Feldhauptmanns "brave Leut" gelelstet habe. Dann unterstatzt er selnen Gedanken mit einem biblischen Zitat ron Jesus, "HelBt's nicht in der Schrift, Was du dem geringsten von melnen Brudern getan hast, hast ou m1r getani"105 D1e Verfremdung dieses Z1tats llegt nloht darin, daB die Form oder die Bedeutung verandert werden, sondern darin, daB das zitat seinen sinn behalt aber trotzden fur die Reohtfertigung elner vollkommen ant1-christlichen Tat wie elner Ermordung gebraucht werden kann. Der ungekehrte Gebrauch, d.h. dle voli1g fremde Um-

105 Hauptmann, IV, 1364. Auf dieses Belsplel we1st Gisela Deblel hin (s. 39). 
gebung, in der sich das Z1tat befindet, wirft Sohatten auf die verallgemelnerte Galtigkelt solcher lebensbestimmenden Autborltaten wie der Kirche, die zusammen mit anderen, po11t1soh-w1rtschaftilchen Elementen dazu befunigt war, ein solches Verbrechen wie den DrelBlgjahrigen Krieg zu begehen und im Namen des Christentums zu rechtrertigen.

Belativierungen durch Nechgatz. Der letzten Gruppe verwandt kommen oft reste Formulierungen vor, dle zwar auch durch den Kontext verfremdet werden, die aber hier nioht durch eine unangemessene Verwendung in Frage gestellt werden, sondern durch einen dem in der Formulierung enthaltenen Gedanken direkt w1dersprechenden Nachsatz. Der verfromdende Angriff konzentriert sich auf die angenomene Bloht1gke1t der Substanz solcher Formulierungen. Im Guten Mensohen kommt an der stelle, wo Wang obdach für die Gbtter sucht, und wo er als Ausrede fur die Ablehnung des Hausbes1tzers Tscheng den Gottern gegentber sagt, Herr Tsoheng wirde zu groBe Furcht vor dem Urtell der Gotter haben, der folgende Dlalog zustande:

Der dritte Gott, sind wir denn so furohterlich?

Wang: Nur gegen die bosen Monschen, nloht wahr? Man we1B doch, daB die Provinz Kwan selt Jahrzehnten von obersohwemmungen helmgesucht wird.

Der zwe1te Gott, So? Und warum das?

Wang: Nun, well dort ke1ne Gottesfurcht herrsoht.

Der zwelte Gott, Unsinnt Well sie den Staudam verfal- 
len lelBen. 106

D1e Redewendung "Gottesfurcht herrscht" w1rd in Gegensatz zu den festen Formullerungen der letzten Gruppe in ablichen Sinne gebraucht. Hier besteht keine Verfremdung durch Veranderung oder falsche verwendung, sondern durch den folgenden Satz, der mit der Selbatverstundichkelt des in der Redewendung 1mplizierten Inhalts schlagart1s fert1g wird. Die Idealisierung des Glaubens elner allmachtigen Gottlichke1t, die die Menschhelt belohnt oder bestraft, je nach dem Vorhandensein einer herrschenden Gottesfurcht, w1rd mit f1naler Geschlossenhe1t als Unsinn entlarvt. DaB elner der Gotter selbst mit lapldarer Sachlichkelt die Scharfe der Bealitat elnfuhrt, verleint dieser auf die cotter bezogenen Verfremdung besondere Betonung.

Nachdem die Katholiken elnen S1eg aber die Evangel1sten darongetragen haben, bel denen slch Anna Flerling und der Feldpredicer aurhalten, sagt der Feldprediger zu der Hutter Courage resignierend. "Wir sind eben jetzt in Gottes Hand", woraufhin die Courage antrortet, "I ch glaub nicht, daB wir schon so verloren sind, aber schlafen tu $1 \mathrm{ch}$ auch nicht nachts." 107 D1e Redewendung in der Aussage des Feldpredigers, die besagt, daB sle alle nichts mehr gegen ihre Lage tun kobnen und quasi nur auf die barmherzige schttzende

\author{
106 Hauptmann, IV, 1491. \\ 107 Ebenda, s. 1378.
}


Maoht Gottes warten konnen, wrd durch Nutter Courages Aussage auf komlsche Welse rerfremdet. Der Feldpredger, der dlese Forwul1erung mit elner Selbstrerstindlichkelt anwendat, 24Bt slah fur den Sohlag offen. Die Bedewendung hat zwal Selten. Elnerselts setzt sie roraus, daB der 3 preoher ol oh In einer aussiohtsiosen s1tuation berindet, aber andererselts wird die beruhigende poaltive Selte (ale Existenz - Ines schatzonden Gotted unmittelbar zum Ausdruok gebracht. Mutter Courages Reaktion daraur bolnhaltet auch diese Doppeleodtigkelt. Elnmal bezieht slch $1 \mathrm{hr}$ satz auf die reale S1tuation, ron der sio glaubt, daB wi nloht allzu sohlim se1. Aber zun andoren hort os siah an, als ob dis Auseage af des In-der-iand-jottes-seln bozogen w1rd, well der Feldprediger duroh selne worte, ohne os zu waen, daraur 2enkt. Sottes Hand wird plotzlich aus den Bereloh des Foaltiven gerissen und in den des Negativen gertoxt. Der Gebrauch dieser leeren phrase, deren elgentlicher Inhalt angeslohto der gesohilderten W1derspriohe dee DrelB1 guhrigen Krieges in der Tet abourd int, wrd auroh ale Relativierung. un es gering anzugeben, in rage geatelit.

Wortilohe Aufremingen. In da lotzten Belaplal glbt o In olnem Punkt olne gewlses Parallele zu den Verfreadungen dieser Gruppe, und zwar duroh die Moglichkelt eines der Formullerung entgegengesetzten Aurfassens des sinngehalts dieser Formulierung. In den nleheten Exompeln aber nutzt Brecht die besenderen ztlge der verblaBten tbertragenen Be- 
deutung 108 der Formulierung vollstens aus. Wle 1 ch schon auf Selte 74 (unten) erwahnt habe, 1st einer der Determ1nanten der Festigkelt einer Formulierung die Starke der Abwelchung der Gesamtbedeutung der Wortverbindung von den urspringlichen Bedeutungen der einzelnen Bestandte1le. Diese Abwelchung kommt durch elne obertragung elnes der Glieder zustande, oder die ganze Wortverbindung kann elne v8111g metaphorische Bedeutung haben. Aber daB die Formulierung Ihre "elgentliche" Bedeutung im Laufe der Zelt verloren hat, 1st uns melotens nicht mehr gegenwartig. Zum Belspiel war "der FuB des Berges" elnmal elne lebendige Metapher, bel der sowohl die elgentliohe Bedeutung des wortes "FuB" (dieser kann in der elgentlichen Bedeutung nur ein Tell des menschlichen Korpers sein) als auch die vbertragung als solche prasent waren. Durch 1hre lang Jahrige Entwlcklung aber 1st dieses metaphorisohe Element so we1t zurackgetreten, dab die abertragene Bedeutung des Wortes sioh zu einer neuen elgentlichen Bedeutung entwlokelt hat. Das he1Bt, "PuB" in dieser Formullerung ist nicht mehr elne vbertragung in dem Sinne, sondern elne weltgehend von dem Menschenkorper losge18ste Bezelchnung fur den untersten Tell des Berges. Die VerblaBtheit von Hetaphern 1st je nach der Entwicklung verschleden stark. In der Belspiel "In der Hand Gottes" hatten wir es mit einer Metapher zu tun, die sich noch im Pro-

108 Ich bezlohe mich auf Walter Porzig. Wunder der sprache, S. 122ff. (Metapher bedeutet vbortragung.) 
zeB des Verblassens berindet. Aber die Verremdung dieser Redewendung warde von Brecht nicht durch eine Ansplelung auf die elgentliche Bedeutung dieser errelcht, sondern durch ein Sp1el mit der Bewertung der durch d1e Metapher ausgedrackten situation. Dagegen werden feste ibortragene Formul1erungen v1e die folgenden duroh ein "elgentliohes" Verstehen verfremdet. E1ne wirksme Verfremdung hier setzt voraus, daB die elgentliche Bedeutung zurtokgerufen und die Metapher weder gegenuixtig als Metapher aufgedeckt w1rd. Gehen w1r jetzt von der Hand Gottes zu der Hand Wangs aber, die euf brutale Welse ron dem relohen Barbler Shu Fu m1t der Brennschere kaputtgesohlagen w1rd. Nachdem Shen Te 1 hm verspricht, sie warde einen Keineld for Ihn lelsten, da die Zeugen dieses Ereignisses zu viel Anget haben, gegen den Herrn Shu Fu auszusagen, verwandelt sich Shen Te in Shul Ta, bevor sle ihr Versprechen elnhalten kann. Ala Wang zu dem Laden Kommt, um mit Shen Te zun Gericht zu gehen, begegnet $1 \mathrm{hm}$ der redegerandte shu1 Ta, der sein Vorhaben zerschlagt. Spater, als Wang die Schlauhe1t Shu1 Tas bezeugen w11. sagt er, "Er 1st ein ungemein geschickter Mensch, Ich habe es am elgenen Le1b erfahren...-109 Im gewohnl1 chen Gebrauch 1st die Redewendung "am elgenen Le1b erfahren/spuren" nicht mehr im elgentilchen physischen sinne gemeint. sondern auf den allgemelnen Berelch des "pergonlichen Erfahrens" tbertragen worden. Die wortwortliche Verwendung 
dleser metaphorisohen Formulierung, die in dlesem Zusammenhang auoh ein elgentliches Auffassen von Leser oder Horer erzwingt, verfremdet die Formullerung und dient dazu, uns auf die gesellschaftilche Ungerecht1gkelt des "BrennschereErlobnl sses" Wangs zurthckzulenken, bel dem der arme Mensch nicht ranig 1st, dem Gesetz folgend gegen den Relchen anzugehen.

D1e Stelle im Guten Menschen, an der wang für die Gotter Unterkunft sucht, erwelst sich als eln richt1ges Konzentrat von sprachlichen Verfremdungen. Hier benutzt Wang -1ne feste Wortverbindung als er einen Herrn anspricht und versucht, Inn zum Aufnehmen der Gotter zu tberreden, "Gre1fen sle un Gottes willon zul"110 Diese sehr gebrduchliche Formullerung "um Gottes w1llen", die in unserem sprachgebrauch volikommen zum lediglichen Ausdruck der Betonung umgedeutet geworden 1 st, bekommt elnen verfremdenden StoB, Indem sie plotzlich in dieser situation of renbar wirklich zutrifft. Die Herren sollen tatskchlich um Gottes willen zugrelfen. Das wortwortliche Auffasen dieses sonst inhaltslosen Betonungsausdrucks verle1ht $1 \mathrm{hm}$ wleder Bedeutung und welst durch die Hervorhebung dieser elgentlichen Bedeutung noch elmal auf die elgentliohe Position der Gbtter, numlich euf 1hre Meohtlosigke1t hin.

Manche Belspiele decken mehr als elne Verfremdungsgruppe. D1e folgende Verfremdung 1llustriert diese Ersohe1- 
nung. Der Koch in Mutter courage abt Kritik am schwedisohen Konig, in dessen Dienst or steht. Dor Feldprediger, der dieser Kritik nloht zustimmt, halt inm ror, "SchlleBlich essen S1e sein Brot." Der Koch sagt daraufhin, "Ioh eB nloht seln Brot, sondern $1 \mathrm{ch}$ beoks inm."111 In diesem Fall sehen w1r, daB die Verfremdung dieser Redevendung " Jemandes Brot essen" sowohl duroh elnen relativierenden Nachsatz als auch durch ein Wortwortlichnehmen vollzogen w1rd. Der 1mplizierten Behauptung des Feldpredigers, nlm$11 \mathrm{ch}$ daB der Koch unverdient auf Kosten des Konlgs lebe, w1rd auf komisehe welse durch den darauf folgenden Satz w1dersprochen. Erstens melnt der Koch, or whrde dooh eine Gegenlelstung erbringen, und zwar duroh seinen Dienst als Koch. Zum anderen besagt die wortllche Aufrassung der Redewendung, daB der Koch, obwohl er als Koch tatig 1st, im D1enst des Konlgs nicht elnmal das Recht hat, das zu essen, was or selbst gebecken hat. In der Bedewendung steokt die selbstverstandlioh gewordene Haltung, daB der "Arbeltgeber" quas1 den gatigen "Brotgeber" verkorpert, D1e Tatsache, daB der Koch (unter zahllosen anderen "Arbeltnehmerm") in diesem Verhaltnis nichts geschenkt bekommt, im Gegente1l, daB er von seinem "Arbeltgeber" nur ausgenutzt w1rd, w1rd an dieser stelle entlarvt.

E1ne weltere mogliche Ansplelung diesbeztglich besteht

$111 \mathrm{G}$. Debiel fuhrt auf $\mathrm{S}$. $41 \mathrm{f}$, dieses Belspiel als Verfremdung elnes metaphorlsohen Zusammenhanges ror. Hauptmann, IV, 1375. 
In der wahrscheinlichen Herkunft dieser Redewendung. EIn altes deutsches Sprichwort lautet, "Wessen Brot $1 \mathrm{ch}$ esse, dessen Lied lch singe."112 Angenommen Breoht warde diesen Spruch kennen, enthielte der Satz in diesem Kontext -1nen welteren verfremdenden Widerspruch. Wenn der Kooh sagte danni "Ich eB nicht sein Brot", bedeutete das, daB er auch nicht "das Lled des Konlgs sänge", d.h. daB er auch nicht seine Prinziplen fur seinen Lebensunterhalt (sein Essen) aufgube. Aber der Koch bewe1st selbst, da er Im Dienste des Konlgs trotz seiner Kritik an diesem tatig 1st, se1ne von inm selbst bestrittene Kafliohkelt. Weiterhin soll diese Stelle auf die gesellsohaftlioh-8konomischen Verhaltnisse hinwelsen, die die Kaflichkelt der Menschen als einzige Moglichke1t des E1nkommens erzwingen.

E1ne der starksten Verfremdungen dieser Gruppe im Guten Menschen 1 st die folgende. Nachdem die Gotter erfahren haben, daB der "Engel der Vorstadte" Shen Te mit dem "Tabakkon1g" Shu1 Ta 1dent1sch gewesen war, begeben sie sioh In die Tuschung, alles sel doch in Ordnung, und wollen offensichtlich den ort so schnell we mbglioh verlassen. As Ende elner klelnen lyrischen Passage, dle von den Gottern gesprochen w1rd, erschelnt diese abschl1eBende Zelle, "Leb wohl, mach's gutiw113 Als normale Absohiedsworte haben

112 Karl Frlearloh Wilhelm Wander, Deutsches Spriohworter-Lexikon (Lelpz1g, F.A. Brockhaus Verlag, 1867), I, 479.

113 Hauptmann, IV, 1605. 
diese zwe1 haufig gebrauchten Formulierungen nlohts besonders Merkwtrdiges an sich. Aber das Auftreten dieser Worte in diesem Sttck und ror allem an dieser Stelle des Schelterns Shen Tes kommt elnem ausgesprochen absurd vor. Die Lacherllchke1t llegt daran, daB die elgentliche Bedeutung der abgeblaBten Redewendungen durch die in versohledentilcher Form oft wlederholte Themat1ki "Kan kann nicht gut sein und auch leben", oder um die in diesem Belsplel erschlenenen WOrter zu verwenden, "Man kann nlcht wohl leben und es auch gut machen," der Leltgedanke des sttickes hervorgehoben wird. Durch das wortwortliche Auffassen dieser Formulierungen wird die S1nnlosigkelt inres Gebrauchs in elner solchen geselischaftichen situation wie der Shen Tes aufgedeckt, um nach Brecht welterhin die S1nnlosigke1ten eines solchen Gesellschartssystems anzuprangern, die te1lwe1se durch die Selbstverstundlichkelt des sprachgebrauchs verschlelert werden.

D1e sprachl1chen Verfremdungen dieser Kategorlo tendieren dahin, die festen sprachlichen Gewohnhelten in Frage zu stellen, oder besser gesagt, Breoht grelft direkt die Formelhaftigke1t der sprache an, well elnerselts durch die Entstehung von sprachlichen Formeln das selbstandige Denken beeintraohtigt w1rd, denn es 1st fur die Menschen lelohter, -1ch in Formeln auszudrtlcken, als jedes wort auf seine Bedeutung hin so auszusuchen, daB es dem Gedanken genau entspricht, und wenn die Gedanken sohon durch festgeprigte 
Formullerungen beelnfluBt werden, orwelst $81 \mathrm{ch}$ das Denken selbst als elngeschrankt. Andererselts hat elne festgepragte Sprache den groBen Nachtell, daB die Pragunzen zu bestimmten Zeltpunkten in der Entwlcklung des gesellschaft11chen Zusammenlebens der Menschen entstanden sind, sie markleren sozusagen gewlsse Zustandlichkelton, Verhaltnisse, Denkwe1sen usw. der Gesellschaft der Entstehungsze1ten dieser Formullerungen, aber da das Zusammenleben der Menschen sich immer welter verlndert und nach Breoht verandern muB, ervelsen sloh die unveranderten sprachlichen Formulierungen zusammen mit inren Inhalten oft als nicht geltend. Aus diesen zwel Selten der formelhaften Sprache ergibt sich, daB in elnigen Fallen falsche oder veraltete Denkwelsen und Authoritaten aufrechterhalten werden, und daB durch die Selbstverstandlichkelt, mit der diese Formulierungen verwendet werden, auf subtile welse viele Ungerechtigkelten in der Gesellschaft versohlelert werden. Manche Formullerungen haben sogar von inrem Ursprung an gesellschaftliche Ongereoht1gkelten verhohlen.

In Brechts Aufsatz "Funf Schwierigke1ton beim Sohre1ben der Wahrhe1t" gibt er selbst einige Belspiele an, wie man durch elne bestimmte Wortwahl "Ilgen" vermelden kann. Zum Belsplel, Man sollte nicht "Volk", sondern "Bevolkerung" sohrelben, denn "das Wort 'Volk' besagt elne gewise Einheltliohke1t und deutet auf geme1nsame Interessen hin", whrend "Bevblkerung" die Wahrhe1t ("rersohlodene, auch 
elnander entgegengesetzte Interessen") ausdricke, die in dem Ersteren unterdrackt werde. Und wo gesellschaftliche Unterdrdokung herrsohe, sollte man naoh Brecht fur das Wort "Disziplin" das Wort "Gehorsam" wahlen, "we1l D1sz1plin auch onne Herrscher moglich $18 \mathrm{t}$ und dadurch etwas Edleres an sioh hat als Gehorsam."114 Hier laBt sich die allgemelne gedankl1che Grundelnstellung ron Brecht beztglioh selner Sprachgestaltung sohen. Auf der elnen Selte 1st es erforderlich, die "Lagen" der gesellschartlichen Verhaltnisse, die durch d1e Sprache masklert werden, zu entlarven. Auf der anderen Seite muB die "Wahrhelt" selbst in der sprachgestaltung dargestellt werden. Das Erstere versucht Brecht durch die oben herausgearbelteten Verfremdungen selbstverstandlich gewordener Formulierungen, Indem er der "Lage" ein Kontra bletet. Das Letztere versucht er weltgehend durch neue verfremdende Formullemingen, Indem er auf direktere Welse versucht, die widersprtchlichen Zustundlichkelten, wle in folgenden Tell dieser Untersuchung, aufzudecken.

Es war meine Absicht in der ersten Kategorie gewesen, zu zeigen, daB Brecht versucht, den Gebrauch solcher Formullerungen durch die oben genannten vler Verf rendungsgruppen abzubauen. Wenn w1r diese dialekt1sche Methodik des InFrage-stellens ${ }^{115}$ aufgrund des von Brecht intendierten Abbauprozesses "destruierend" nennen, dann gilt es, die ver-

$$
\begin{aligned}
& 114 \text { Hauptmann, XVIII, } 232 . \\
& 115 \text { Deblel, S. } 45 .
\end{aligned}
$$


fremaungen der nalohsten Kategorle "konstrulerend" zu nennen. Brecht baut hier kelne alten Formulierunsen ab, da er stattdessen in verfremdender Welse neue "wahrhe1tsenthaltende" Pormulierungen aufbaut. Belde Methoden dienen dazu, die Menschen zu der BewuBtwerdung lhrer Sprache zu erz1ehen, damit sie schlieBlich zu der BewuBtwerdung Ihrer elgenen sesellschaftlichen Verhaltnisse kommen. Aber hier 1st das dialextische Verfahren eln anderes. In der vorigen Kategorle stellt Brecht der sohon bestehenden Formullerung (These) elne Antithese entgegen. In der nachsten Klasse verfremdet er die gewohnliche Sprach- und Denkordnung, Indem er beldes: These und Antithese in elner neuen Formullerung 11efert, oder 1ndem der den Leser/Horer bereits mit einer Synthese versorgt.

\section{Verfremdung durch noue verfremdende Formulierungen}

Im letzten Te1l habe $1 \mathrm{ch}$ von Formulierungen gesprochen, denen elne sewisse Festigkelt in der Wortverknuprung innewohnt. In diesem Tell 1st es melne Intention, sprach11ohe Verfremdungen zu zelgen. die in den Bereioh der "rrelen" Wortverbindungen fallen.

Bel freien oder unfesten Wortrerbindungen 116 ist die Gesamtbedeutung aus der Summe der Elnzelbedeutungen zu ersohl1eBen. Das he1Bt: Die Bedeutungen der elnzelnen GI1eder beeinflussen sich in der Verbindung nicht gegeneeitis.

\section{Agricola, S. 588.}


$O b$ alleinstehend oder in der Verbindung, haben die worter dieselben Bedeutungen. Bel festeren Wortverbindungen haben w1r gesehen, daB gerade durch die besondere Wortverkmaprung elnzelne Bedeutungen sich verandern. Belspielswelse Abernimmt das Wort "we1B" in der Rederendung "der we1Be Tod" durch den ElnfluB des Wortes "rod" die bllahaft derstellende Funktion elnes Todes durch E1s und Schnee. Frele Wortverbindungen dagegen ("elne groBe/gelbe/nasee Banane/Zahnburate" oder "lelse/kaum/spater beruhren/sprechen") sind allein duroh den selbstandigen sinn der Bestandtelle und durch d1e auf diesen selbstandigen sinn der Bestandte1le basierende Absicht des Zusammenstellens zu begrtinden. EIn "blinder Alarm", haben wir gesehen, ist nioht ein Alarm, der ulrkl1ch blind 1st. Dagegen 1st eine "nasse Banane", obwohl dies elne selten erscheinende Wortverbindung 1 st, elne Banane, d1e tatadchlich naB $1 \mathrm{st}$.

Also sind rrele Wortverbindungen einzig und aliein durch die grammatisohe und die semantische Kongruenz begrandet. D1es bedeutet: Solange die Wortverbindung eine sinnvolle grammatiache struktur (nicht, "das Aprel rallen". sondern "der Aprel fallt) und einen neiteren bedeutungsmaB1gen Sinn (nicht: "d1e Kuh 1Bt", sondern "d1e Kuh rriBt"17) hat, braucht sie keine welteren Bedingungen zu erfalien, wie etwe 1rgendwelche rerbindungsberest1gende Bestimmungen. Im

\section{Le1s1. S. $68 \mathrm{ff}$. Andere Ausnahmen sind in Le1818} Behandlung der "direkten Hetaphern" zu finden. 
Gegentell sogar, sle 1st, wle gesagt, Innerhalb inres grammat1sch-semant1schen Rahmens voll1g frel und unfest.

Aufgrund der zwel Grenzen (der gramatisohen und der semant1schen), die bel der sinnvolien Sprachgestaltung gezogen werden, ware es theoretisoh mbglich, verf remdende Wortverbindungen $z u$ konstruleren, Indem destrukt1ve Versuche gerade mit der grammat1schen und semant1schen struktur gemacht werden konnen. Da es aber nie Brechts Absicht gewosen 1st, gramatisoh und semantisch unsinnige Wortverbindungen zu bauen, lasse $1 \mathrm{ch}$ dleses mbgl1ch verfremdbare Geblet in Ruhe.

Was glbt es denn sonst in der Sprache, was so verfremdber 1st und was Brecht zu verfremden versucht? Denn d1e sohopfung neuer verfremdender Formullemangen setzt voraus, daB 1rgendetwas (etwas Ungewohnliohes) in der Sprache vorkommt. Und etwas Ungewohnllohes setzt wiederum roraus, dab bestimmte Gewohnhelten in der dblichen Sprachgeataltung vorllegen, die durohbrochen werden. Also: wenn es nioht die Grenzen betrifft, die notwendig fur die Bewirkung einer grammatischen und semantischen Verstandigung sind, muB dieses Etwas andere Sprachgrenzen tbersohrelten.

Diese Grenzen sind auBerst unklar und lassen sich sohlecht unbestritten festaetzen. Elne dieser Grenzen betrifft die st111st1k, oln fast untbersiohtliches Thema und eine Untersuchung ftor sich. Die andere Grenze, die uns hier interessiert ist genau so unfestlegbar und betrifft einen 
noch untbersehbareren Gegenstand, namlioh die Gewohnhelt in Denken.

Offensichtlioh 1 st es Innerhalb des Rahmens dieser Untersuchung ausgeschlossen, diese belden Geblete in Ihrem Umfang zu behandeln. Deswegen 1st es notwend18, diese Untersuchung der verf remdenden sprachgestaltung Bertolt Brechts auf die Heupterschelnungen beztigl1ch der zwel erwhnten Themen elnzusohranken, und dies auf el ne solohe Welse, daB far das Verstandnis dieses Tells der Arbelt nur eln Minlmum an wissenschartliohem Elngehen in die Sache orforderlich 1st. Ea lat sowieso fraglich, inwlewelt elne tlefere wissensohaftliche Forsohung dieser zwel Geblete dazu beltragen warde, die Absichten dieser Untersuchung zu real1sieren. Melnes Erachtens gentgt es für unsere Zwecke vollkommen, zu wissen, wo und wann Verfremdung dle belden Berelche berthrt. Es lst ohnehin elne sehr vage Angelegenne1t, Grenzen zu setzen, die markieren sollen, wo st1list1sohe oder sedankliche Gewohnhelten gestort werden, da eine unzahlbare Menge von bestimmenden Faktoren im Spiel sind, w1e z.B. Alter, B1ldung, Beruf, Klassenschioht des Horer/ Lesers und sogar die Landsohaft, in der er aufgewachsen 1st, um eln paar allgemelne zu nennen, ganz von unterschledlichen Individuellen EInflassen Innerhalb dieser Lebensbereiche zu sohwelgen.

Also bleibt mir in dieser Untersuchung nichts Anderes ubrig, als elnerselts von der sogenannten "Intuition" aus- 
zugehen, das bedeutet im Hinblick auf die Verf remoung, eine Gewohnhe1tsstorung da festzulegen, wo me1n "Sprachgefthl" es mir diktiert, und andererselts von meinem Brecht-Verstandn1s auszugehen, Insorelt ich imstande bin, elne Realisierung selner W1rkungsintentionen in seiner Sprachgestaltung zu erkennen.

M1t diesen Gedanken als Unterlage mbohte 1ch jetzt mit der E1ntellung dieser Kategorie beginnen und diese mit Beispielen unterstatzen.

Scheinbar unangemessene Verbindungen zweler Begriffsfelder. Jeder Mensch denkt in Begriffen, die er auf seine Welse (melst sloh dessen nloht beruBt) bewertet und kategor1siert. Versuche, die worter best1mmten "Feldern" zuzuordnen, werden in der modernen Sprachwissenschart intensiv erortert. Wie diese Begriffe genau klassifiziert werden konnen und wo die doutlichen Grenzen zwischen elnem und elnem anderen Feld liegen, ist eln nooh ungelostes Problem der Semantik, und elne zufriedenstellende Losung zu der sehr unstrittenen Frage, ob es uberhaupt moglich 1st. Begriffe zu kategorlsieren, 1st wahrschelnlich noch nlcht gegeben worden. DaB es Begriffe tberhaupt gibt, 1st aber, sowe1t 1ch informlert bin, noch nioht abgestritten worden. Und ob man eine ausrelchende Kategorislerungsmethode findet oder nicht, dies verandert nicht die Tatsache, daB die allgeme1ne Blohtungsilnte dieser Versuohe schon zu vielen rlohtigen Ergebnissen gefuhrt hat. 
Wenn 1ch also den Terminus "Begriffsfeld" von dleser Abtellung der Sprachwissensohart mit elnigen Bedenken tbernohme, 1st dies keln Hinwels darauf, daB loh damit bestimnte spez1f1sche sprachw1ssenschaftliche Theorien vertrete, sondern lediglich elne Bezelchnung fur eine theoretisoh mogl1che, allgemelne Begriffsgruppe, wie z.B. elne Positir-Negat1v-Neutral-Grupplerung .

Brecht erzeugt oft verfremdende Wortkombinationen, 1ndem or zwel mitelnander ansoheinend unvereinbare Begriffsfolder in einen Zusammenhang stellt. Dies macht or mit der Abs1cht, gewisse "Wahrhe1ten" oder "Real1tuten" In der Gesellsohaft zu entlarven ${ }^{118}$, d.h. gewisse mltelnander unverelnbare, widersprtichliche Verhaltnisse zu zelgen, die normalerweise nioht in der Sprache zum Ausdruck kommen. Schon auf Selte 62f. dieser Untersuohung habe 1ch ein passendes Belsplel dieser Gruppe angefuhrt, namlion die Frage Shul Tas, "Wle soll man sich von allen Schwlchen frelmachen, vor allem von der todlichsten, der Llebe?" Da $1 \mathrm{ch}$ diese Verbindung dort schon erlautert habe, brauche $1 \mathrm{ch}$ es hler nicht mehr zu tun. Elnen ahnlichen Fall gibt es aber 1m Kaukas1sohen Kre1dekre1s. Der Palast des Gouverneurs 1st aberfallen, zerstort und im Tumult verlassen worden. Aber das Kind der Gouverneursfamille hat man vergessen. D1e Magd Grusche blelbt trotz der Gefahr bel dem K1nd zu-

118 Hauptmenn, XIX, 326. Brecht sohre1bt, "Real1st1sch he1Bt, den gesellschaftlichen Kausalkomplex aufdekkend / die herrschenden Gosichtspunkte als die Gesichtspunkte der Herrschenden entlarvend..." 
raok und wartet bis jemand kommt, es abzuholen. Aber wahrend der ganzen Nacht erscheint niemand. In der Ze1t whohst Ihr das Kind lmmer mehr ans Herz. Sie holt Milch fur es und hallt es warm in einen Brokatmantel eln. Der sunger, der dies sohlldert und der die Gefthle der Grusohe verfolgt, sagt dann, "Schreoklich 1st die Verfunrung zur Gatel"119 DaB der Begriff "Gtite", der in der gewohnliohen Denkhaltung durchaus ein Positivum 1st, plotzlioh in der Sprache als "Verfuhrung" auftaucht, verfrendet den Horer/Leser, der das Wort "Verfuhrung" zu dem Berelch des Negativen zuhlt. Dieses Zusammenzlohen ron zwel sohel nbar gegensutzlichen Bewertungsberelohen widerspricht der gewohnliohen Denkwelse und damit auch der gewohnlichen wortzusammenstellung. Doch w1ll Brecht gerade die Widersprtchl1ohkelt elner "Heal1 tat" zeigen, und zwar elne doppelte Widersprtichliohke1t. Erstens 1st die S1tuation insofern an sich widersprichlich, als Glte tatskohlioh unter bestimmten gesellsohaftlichen Verhaltn1ssen "sohreckl1ch" und gefahrlich seln kann, und zweltens 1st es eln Widerapruch, daB diese Verhaltnisse aberhaupt ex1stieren können, wenn sie der gewönnlichen Denkweise nicht entsprechen. W1r halten es in unserem Denken ftur ungewohnlioh, Gtte als Negativum gebraucht zu horen, doch nach Brecht llegt dlese Begriffsverblndung in der Realltat berelts auf der Hand. Fast auf dieselbe Welse wird dies 119 Eauptmann, V, 2025, G1sela Debiel welst auf dieses Belspiel hin (S. $45 f^{\circ}$ ). 
1in Guten Mensohen mit einer von Shen Tes Anreden ans Pub$11 \mathrm{kum}$ ausgedrackt, "Welohe Verfahrung, zu schenken I"120

E1n anderes Be1spiel 1m Kaukag1sohen Kreldekre1s gibt auch diesmal der sunger, der die Praxis des Rlchters Azdak in worte raBt. Der Riohter dreht alle Gesetze um, so daB des, was vorher als gesetzmabig galt, verurtellt wird, und was vorher Unrecht war, zum Recht wird. Auf diese Weise baut or eine Art Gerechtigke1t ith die Armen auf. Der Sanger: "Und so brach er die Gesetze wie ein Brot, daB es sie letze..."121 Eine in der gewohnliohen Denkwelse als Negativum bewertete Thigke1t des Gesetzbrechens wird mit dem ursprtinglich ohristlichen Begriff des "Brotbrechens" In elner Formullerung glelchgesetzt. Brecht w1ll auf den widersprtohliohen Gesellsohaftskomplex hinweisen, In dem diese sloh im gewbhnlichen Denken berindende, w1derspreohende Begriffskonstellation eine Realitut 1st.

Im Guten Kengchen sagt der 1 , Gott zu dem verzwe1felnden Wang, "Die Lluse und die Zwelfel haben dioh halb aufgefressen, scheint es."122 Hier stellt Brecht "Lause" und "Zwelfel" In eine zumindest seltene Partnorschaft. DaB diese belden Begriffe diegelbe Funktion (nlmiloh das "Auffressen" von Wang) erfullen, verfremdet uns insofern, als

120 Hauptmann, IV, 1570.

121 Ebenda, V, 2086. G1sela Deblel welat auf diesea Belsplel hin (s. $49 f$.$) .$

122 Ebenda, IV, 1564. 
s10 in unserem gewohnlichen Denken gar nichts miteinander zu tun haben. Belde Begriffe sind zwar als negative in unserem Denken elngeordnet, aber der zwelfel gehort zum gelst1g-psychisohen Berelch, whrend "Lluse-Haben" elnon physischen Zustand kennzelchnet, der tberdies nur bel den Armen vorgefunden wind, Diese Zusammensetzung soll den Horer/Leser dazu anregen, sloh zu fragen, warum diese zwe1 schelnbar unangemessenen Berelche nebenelnander gertokt werden. Wang fungt gerade deswegen an, zu zwelfeln, well seln restes Glauben an das Eingreifen der Gotter sich nloht bewahrheltet hat und die materielien Verhultnisse nloht verandert hat. D1e Armen haben trotz ihrer Horfnung auf die gottliche Hilfe lmmer nooh sozusagen die Lause, die die Gotter wohl selbst sehen, jedoch in elgentlichen sinne nicht aur diese Tatsache elngehen, denn sie beschartigen aloh ja nlcht mit dem materielion Problem.

Als Shen Te tberlegt, ob sle es lelsten kann, den Gottern Unterkunft zu geben, halt Wang ihr vor, daB man in so olnem Augenbliok nioht rechnen durfe. Daraufhin sagt s1e1 "Ich welB nloht, der Magen knurrt lelder auch, wenn der Kalser Goburtatag hat."123 Be1m Horen oder Lesen dieges Satzes befindet man sich in der Lage, die ungewbhn11 che Verbindung zw1schen dem knurrenden Magen und dem Geburtstag des Kaisers nachvoliziohen zu mussen. Hier 1st wleder elne scheinbar unangemessene Verknupfung von zwe1

123 Hauptmann, IV, 1494. 
Begriffaberelchen gegeben, die auf ein reales D1lemme in der Gesellschaft hinfuhren soll. Erstens stellt dieser Satz die 1deal1sierte Erhabenhe1t der Gotter und des Ka1sers In Fragel denn trotz dieser 1st die materielle situation der Armen so, daB Hunger herrscht. Deutlich wird der widerspruch, daB manche hungern, whrend andere sich den Luxus einer extravaganten Geburtatagareler leisten kbnnen. Zweitens deutet diese Begriffererbindung an, dab ein solcher Luxus sogar gerade deswegen moglich 1 st, well die Mkgen anderer knurron. Die Polaritat zwisohen den Armen und den Relohen wird duroh elne solche Formullerung hervorgehoben, um die Ungerechtigkelten elnes geselischaftlich-okonomlsohen Systems zu zelgen, in der es elne herrschende relche Minderhe1t und eine rerknechtete arme Mehrhelt gibt.

Ein anderes Belspiel rattert Shul Ta auf drelfache We1se herunter, wenn er aber shen Te sagt, "Man hat a1e besohuldigt, gehungert zu habenl Es 1st notorisoh, dab s1e in Armut lebte. Inr Leumund lat der allersohlechtester es ging Inr elend / 124 DaB hungern zu massen ein verbrechen 1st, daB Armut in Bekanntsein (m1t negativem Belgesohmack) herbelrurt, und daB Elend elnen schlechten, sogar den "allerschlechtesten" Leumund kennzelchnet, sohelnt uns Hörern/ Lesern eine Absurditat zu seln. Sprachlich kommen diese Berelohe in log1scher Verkntprung verfremdend ror. Aber dam1t w1Il Breoht sagen, daB, obwohl diese Konstellation im 124 Hauptmann, IV, 1516. 
Gerohnlichen abstoBend emprunden wr, es in der Realitat, in Handeln der Menschen anders aussieht.

E1ne andere Art Verfremdung, die auch noch zu dieser Gruppe gohort, sind die schelnbar unangemessenen Verbindungen zw1schen Person- und Sachwelt. Zum Belspiel, Der 1. Gott sagt, als er Wangs doppelbodigen MaBbecher entdeokt und damit auch Wangs Nicht-Gut-Sein erfahrt, "Schon, er fallt weg. Aber was 1 st das schon, wenn einer angefault $18 \mathrm{fl}^{125}$ In der gewohnlichen sprachgestaltung verwenden wir das Verb "anfaulen" melst in bezug auf Sachen, zwar organische Sachen wie Apfel z.B., aber nie auf Menschen. Dies erinnert an Mutter Courage, wo in Titel der ersten Szene der folgende Satz erscheint, "Der Marketenderin Anna Flerling, bekannt unter den Namen Mutter Courage, kommt ein Sohn abhanden." 126 Solche metaphorlsohen Wendungen werden von Brecht benutzt, um auf die Bkonomisohe Unterlage des Gesellschaftssystems hinzurelsen, und um die widernattrlichkelt elner solchen Gesellschaft aufzuzelgen, in der d1e Menschen nicht mehr als Menschen betrachtet und behandelt werden, sondern als Sachen, als Waren. So sohr uns solche Formulierungen ungewohnlich vorkommen, splegeln sie nach Brecht elne widersprachliohe gesellschaftliche Real1tat wider. 127

125 Hauptmann, IV, 1492 .

126 Ebenda, S. 1349.

127 Diese Okonomische Basis der Gesellschart wird 
Verbindungen zweier direkt gegengatzlicher Begriffe. Verfremdungen in dieser Gruppe hneln denen in der letzten Insorern, als in elner Verbindung zwel sich soheinbar gegenselt1g widersprechende Begriffe oder Begriffsfelder vorhanden sind, die dazu dienen sollen, durch eine Gewohnheltsstorung in der Denkwel se entsprechende Widersprache in der Gesellschaft aufudecken. Aber hier in dieser Gruppe llegen die belden Pole der Verbindung nooh welter auseinander, und zwar sind sie nicht nur soheinbar unangemessene Verknupfungen, sondern schelnber direkt gegensatzliche, gegenseltig ausschlieBende Begrifre.

Im Vorspruch des Sttckes D1e Ausnahme und die Regel warnen die Spleler das Fublikum davor, die Geschehnisse in Stlok nioht natirlioh zu finden,

denn nichts werde naturlioh genannt In soloher Zeit blutiger Verwirrung Verordneter Unordnung, planmaBiger W11lktr Entmensohter Mensohheft damit niohts Unveranderlich gelte, 128

Im Stilwortschatz nennt sich elne solohe Formulierung wie

von Brecht auch da dargestellt, wo Wang versucht, die Herren zum Aufnohmen der Gotter zu tberreden. Selne Sprache 1st ausdroklich die elnes StraBenverkdurers, wenn or sagt: "Gehen sie nicht welter! Jberzeugen S1e sich selberl Ein Blick gentgti Grelfen Sie um Gottes willen zul Es ist elne elnmalige Gelegenhelt! Bitten Sle die Gotter zuerst unter Ihr Dach, bevor sle Ihnen jemand wegschnappt, sle rerden zusagen." (Hauptmann, IV, 1493.) Die Gotter werdon prakt1soh als Marktartikel verkaurt.

128 Hauptmann, II, 793. G1sela Doblel welst auf dieses Belspiel hin (S. 62). 
"verordnete Unordnung" elne "contradictio in adjecto", in welcher die im Belwort ausgesagte Bescharfenhelt in W1derspruch zu 1hrem Hauptbegriff steht.129 H1er w11l Brecht zelgen, daB es nur die herrsohende Klasse 1st, die 1hr Gesellachartssystem als in ordnung bezelchnen marde, daB aber In der Real1tut diese "Ordnung" gegen jede naturliche ordnuns steht und elgentlich deswegen Unordnung 1st, die bloB den Schein von Ordnung besitzt. Annlich verhalt es sich bel "plarmaB1ger W1Ilkur". D1e W1Llkar der herrsohenden Klasse gegen die Ausgebeuteten wird ron den Ausbeutern naoh Plan betrieben und sogar gerechtfertigt, 130 Die Bedeutung ron "entmenschter Menschhe1t" 18t aus sloh selbst zu erschlleBen.

Der Sunger 1m Kaukag1sohen Kre1dekre1s stellt die folgenden zwol Fragen!

Die stadt llegt stille, aber warum gibt es Bewaffnete? Der Palast des Gouverneurs llegt frledl1ch

Aber varum ist or eine Festung 131

Die Vorstellung elner stilien stadt und elnes frledilohen

129

$$
\text { w11pert. S. } 542 \text {. }
$$

130 Hauptmann, II, $807 f$. Der Kaurmann in DIe Ausnahme und die Regel singt, "Der kranke Mann stirbt und der starke Mann ficht/ Und das 1st gut so./ Dem Starken wird geholfen, dem Sohwachen hilft man nicht/ Und das 1st gut so... / Und der Gott der Dinge, wie sie sind, schur Herr und Knecht I/ Und das war gut so./ Und wom's gut geht, der 1 st gut, und wen's schleoht geht, der 1st schleoht/ Und das 1st gut so."

131 Hauptmann, V, 2013. G. Deblel welst auf dieses Belspiel hin (s. 51). 
Palasts wird in verf remdender Welse dem B1ld elner bewaffneten stadt und elner Festung gegentbergestellt. Hier sind die elnander ausschlieBenden Worter streng genommen nicht In Form der "contradictio in adjecto" gestaltet, aber sie berirken durch die paradoren Zuge der Zusammenstellung das glelche verfremdende Resultat. Durch die Verbindung scheinbar gegensatzlicher Beschrelbungen werden die stille und der Frieden als betrigerisch aufgedeckt, elne krlegerisohe Situation wird angedeutet.

Verfreadende Verbindungen zreler sich direkt unterstatzender Besriffe. Den Verfremdungen der letzten Gruppe stehen die Verbindungen dieser gegentber. Was in dieser Gruppe ungewbhnlich erscheint, 1st die Wlederholung, die st111st1sch gesehen elnen stutzig wacht. Melstens bestehen diese Formulierungen aus zwe1 atamglelohen wortern, bel denen das Verb und sein "Inneres Subjekt" oder das Verb und seln "Inneres objekt"132 zum Ausdruck kommen. In der Drelsroschenoper steht als erste Beschrelbung des Buhnenortes: "D10 Bettier betteln. die Dlebe stohlen, die Huren huren. E1n Morttatenalinger alngt eine Moritat."133 In den ersten dre1 Verbindungen betrifft die wiederholung das verb und sein inneres subjekt. Das Subjekt, das schon praktisoh in Verb selbst impliziert 1st, ohne daB es zum

132 Volker Klotz, Bertolt Brecht: Verwoh aber das Werk (Bad Homburg V.D.H.. Berlin, Ztrich, Verlag Gehlen, 1967), S. 107f. Auch Deblel, S. 58fr.

133 Hauptmann, II, 395. 
Ausdruck kommen muB, wird trotzdem versprechlicht, was zur Folge hat, daB der Horer/Leser eine storung emprindet. D1e storung kommt zunllohst durch das Aussprechen ron etras, was schon $\nabla 011$ ig selbstverstandlioh ist, zustande. Aber gerade diese Selbstreratundlichkelt zusammen mit bestlmmten st111stischen Vorstellungen entzieht uns in der gewobnliohen Sprachgestaltung die volle Gegenwlitigke1t der Bedeutung des Ausdrucks. Um unser St1lgefthl zu befrledigen, versuchen wir melstens, solche wiederholungen zu vermelden, indem w1r Umschreibungen konstruleren, wie das folgende Be1opiel, das Gisela Debiel anfuhrt, "Die Bettler bitten um Almosen."134 Aber sobald elne solche Formulierung rorkommt, bekommt das Ganze elne vollig andere Perspektive. "Um AImosen b1tten" ruft die Vorstellung von elnem Gnadenakt herbe1. der Brecht gerade duroh selne Pormullerung vorbeugt. Der Horer/Leser w1rd durch d1e Quas1-W1ederholung gezmungen, bel der Bedeutung zu verwellen, dan1t der reale, unwardige gesellsohaftliche Zustand elne Chanoe hat, sein wahres Geslcht zu ze1gen. Der Bettler b1ttet nach Brecht nicht um Almoseni der Bettler bettelt. Bel der Formulierung, "eln Moritatensinger singt eine Moritat" sind alle dre1, Verb, Inneres Subjekt und Inneres objekt bete111gt. Im Badener Lehrstuck rom E1nverstundnis sagt der gelernte Chor zu den drel gesturzten Fliegerm.

$$
134 \text { Deblel, s. } 58 \text {. }
$$


Erhebt Euch

Sterbend euren Tod w1e

Ihr gearbe1tet habt eure Arbe1t

Umulizend eine Umullzung. T35

Diese sind alle Belspiele des rersprachlichten inneren Objekts.

Andere Beispiele dieser Art befinden sich im Guten Menachen. Shen To LuBert sich Her Suni "E1n Flieger muB rllegen."136 sie sagt an Ende des sttickes zu den Gottern.

Verdammt mich, alles, was $1 \mathrm{ch}$ verbrach Tat 1ch, melnen Nachbarn zu helfen Melnen Geliebten zu lieben und Melnen kloinen Sohn vor dem Mangel zu retten. 137

In Suns "Lled rom Sankt Nimmerle1nstag beschrelbt or, we es an diesem Tag sein warde. In der Aufzahlung komm diese Zelle vor' "Und Verdienst und Verdienen, die machen gute Hienen. 138 Hier ersohelnt elgentiloh das Verb ("verdienen") als Substant1v; aber d1e Funktion 1st dieselbe wie die des Verbes. Alle Verfremdungen dieser Gruppe betonen auf thre Welse und in Verbindung mit ihren spezifiachen Fabeln die Grandbedeutung elnes Begriffs, un deutlicher die damit verbundene Realitat in inrer gesellsohartilohen Relevanz zu sch1ldern.

D1alekt1sohe Darstellungen elnes elnzigen Besriffe.

Brecht varilert das dialekt1sohe Verfahren in selner Sprach-

135 Hauptmann, II, 610 .

136 Ebenda, IV, 1538.

137 Ebenda, s. 1604 .

138 Ebenda, S. 1562 . 
gestaltung, Indem or stattdessen, dab or zwel getrennte Worter oder Begriffsfelder elnander gegentberatellt, einen elnzigen Begriff an rerschiedenon Stelien mit gegensltzlichen Auffassungen versieht. Er tut dies, wle 1ch schon oft in dieser Untersuchung ausgedrlokt habe, um die gesellachartliche Wahrhelt an den Tag zu bringen. In diesem Fall werden die falsohen, sohlelerhaften Gebrache dor Worter durch den fur Breoht "rioht1gen" Gebrauch mit Hilfe der Fabel ausgeschaltet.

Um dies zu lllustrieren, welse $1 \mathrm{ch}$ nooh olnmal auf die Stelle im Guten Mengohen hin, wo Frau Yang ron 1hres Sohnes Besserung erzahlt, wle er "aus einem yerkommenen Mensohen in elnen natzlichen verwandelt rurde." Der Gebrauch des Wortes "verkommen" besagt hier, Yang Sun sel vor seiner Aufnahme in die Fabrik verkommen geresen und danach nloht mehr, Q.h. naohdem der selnen Aufstieg in der kap1talist1schen Hierarch1e begonnen habe. Sohlagart1g dagegen ortont die auf Yang Sun bezogen Anklage des Arbeltslosen 1m Gerichtslokal, "Er 1st der sohlimmste Antrelber, den es je gegeben hat. Er 1st ganz zerkommen. "139 Hierduroh wird es klar, wenn nicht schon an frtherer 3telle, daB Yans Suns "ehrllche Arbelt" selne Verkommenhelt nlcht aufgehoben hat, sondern inm lediglich eln moht1geres Mittel fur den Ausdruck solner Verkommenhelt segeben hat. In Grunde sind os wahrschelnlioh nicht zwel verschledene vorstellungen von 
"Verkommenhelt", die gezelgt werden, sondern zwel sloh gegenseltig widersprechende Vorstellungen von "N1cht-Verkommen-Sein", von denen nur elne rlohtig 1st, namlioh die letztere. Aber letztlich hat der Zuschauer sich zu fragen, warum Yang Sun Uberhaupt "verkommen" 1st. Nach wie vor se1ner Verwandlung 1st er durch die gesellschartlichen Verhaltnisse fast gezwungen, schlecht zu sein.

Da im Guten Menschen das Sttlck s1ch wesentlich mit dem Begriff "Gate" beschuftigt, 1st es deswegen n1cht therraschend, daB auch dieser in elner widersprtichlichen Mehrdeutigkeit auftritt. Er ersoheint sogar so oft, daB eine ganzliche Aufzahlung hier maBig ware. Es lohnt sich aber, elnige Stellen zu erlutern.

Auf der Selte der herrschenden Klasse treten die folgenden zitierten Satze auf (Polizist) "Sie sind sehr gtig. Herr Shu1 Ta."I (Frau Yang) "Tausend Dank, Herr Shui Tal sie sind unendlich gt18..."I (Frau Yang ther Shu1 Ta) "Be1nahe ohne jedes Zutun, aber mit Strenge und Welshelt hat er alles Gute herausgeholt, was in Sun steckte."I (Shul Ta zu Yang Sun beztiglich der Motivation, warum er Sun in die Firma aufgenommen hat) "Um dich zu bessern! Um dich zu bessern!" Bel diesen Belsplelen fallt auf, daB der Begriff "Glte" der Vorstellung eines respektablen kapitalistischen Bargers entspricht, der etwa den standpunkt des Kaufmanns in Dle Ausnahme und die Begel vertritt, "Wem's gut geht, der 1st gut; und wem's schlecht geht, der 1st sohlecht."140 
Diesem Gebrauch gegentber stehen andere Best1mmungen des Gut-Seins, we im folgenden Vers ron Shen Ter "Keinen verderben zu lassen, auch nicht sioh selber/ Jeden mit Gluok zu erftzlen, auch s1ch, das 1 st gut."141 D1ese Quas1-Der1nition des Gut-Seins, we Brecht os in diesem Stbok elgent$11 \mathrm{ch}$ melnt, versucht Shen Te zu realisieren, Obwohl es $1 \mathrm{hr}$ schlecht geht ("Es geht mir nicht gut, und wenn 1 ch bis morgen frah melne Mlete nicht zusammen habe, werde 1 ch hinausgeworfen."), nimat sie die Gotter zu 1 hrem finanziellen Verlust auf. Danaoh bestutigt Wang Shen Tes Gut-Sein. "sie 1 st der beste Mensch von Sezuan." Aber diese Rlchtung, die Shen To als Ideal vertreten soll, wird ron den Herrschenden unmbglich gemecht. Shen To, die "nicht nein sagen kann". acheltert gerade an der christlichen Nachstenllebe ("Du sollst delnen Nahsten I1eben, wie dioh selbst." Lukas 10, 27). Der Charakter dieses 31 oh-Gegenseltig-AusschlieBens der dialekt1schen Ersohelnung geht in diesem Sttck sogar so we1t, daB, um zu leben, Shen Te slch in zwe1 Figuren spelten muB. D1es 1st aber nloht als psycholog1soher Zw1espalt zu verstehen, sondern als sozialer. D1e Figur Shen Te/shul Ta 1st ein Gleichnis fur die Gesellschaft, in der das eine, falsohe "Gute" (Kap1talismus) das andere, richt1ge "Gute" nicht vertragt und deswegen ausrottet. Wenn man sioh der

\footnotetext{
140 Hauptmann, II, 808 .

141 Ebenda, IV, 1553.
} 
w1dersprtchllchen Mehrdeut1gkelt dieses Begriffs bewuBt wird, konnen solche sprliche wie der folgende von den Gottern, die selbst nicht die Problemat1k dieser W1dersprilch11chke1t erkennen, nur als Absurditaten und Banalitaten auftreten. "Sel nur gut und alles wird gut gehen." 142 Sogar der relche Herr Shu Fu drlckt, obwohl unreflektiert und oberflachl1ch, den Unterschled aus, wenn er in seiner tbergchwanglichen Art ther Shen Te sagt, "...der Zauber Fralule1n Shen $\mathrm{Te}^{\prime} \mathrm{s}$ besteht kaum in der Gute 1hres Ladens, sondern in der Gtte Ihres Herzens." 143

In diesem letzten Tell dieser Untersuchung habe $1 \mathrm{ch}$ versucht, Brechts Prinzip der Verfremdung in selner Sprachgestaltung in Ausgang ron seinen Stflcken (hauptsachlich von dem Guten Menschen von Sezuan) aufugliedern und durch Be1spiele zu verdeutlichen. Wie immer, basiert die Verfremdung, so wie Breoht sie zur Geltung bringt, auch in der Sprache auf elner storung einer selbstrerstandlichke1t. ob die Selbstrerstandlichkelt sich in einer festgepragten sprachlichen Formulierung oder in festen Denkmustern man1festiert, die bestimmte Gewohnheitsgrenzen in einer sonst "frelen" Sprachgestaltung setzen, hat sie sozusagen elne doppelte Perspektive, die uns fur die Zwecke dieser Untersuchung interessiert hat, elnmal von Brecht ausgehend die

142 Hauptmann, IV, 1605.

143 Ebenda, S. 1547. 
Notwend1gke1t, diese Selbstrerstandlichke1t zu verfremden, zum anderen d1e Kogliohkelt, dieses Verfremden in der sprachgestaltung zu voliziehen. Die erste selte wurde ausfthrilah genug zu Beginn es zwelten Telles (Verfrendung) als AlIgemelnes behandelt, so daB in sprachlichen Te1l nur HInwe1se auf dieae seselischaftliche flohtung notig waren. D10 zwelte Perspext1ve interessierte uns spezirischer im dr1tten Te11, um zumindest Im Grundprinz1p zeigen zu konnen, wie Breoht versuoht hat, in selner spraohgestaltung gelne geselisohaftilohen Intentionen zu verwirliohen. 
Agricola, Erhard. Die deutsohe Sprecher Klelne Enzyklopedie in zwe1 Bunden. Hrsg. Erhard AGr1001a, Wolfgang Fle1scher und Helmut Protze. Lelpzig, VEB B1bliograph1sches Inst1 tut, 1969.

Ar1stoteles. Poet1k. Hrsg. Olof G1gon. Stuttgart, Ph1l1pp Reolem Jun.. 1969.

Arntzen, Helmut. "Kombdie und eploches Theater." Deutgohunterricht, Jg. 21, Hert 3 (Jul1 1969), s, 67-77.

Bentley, Er10. "D1e Theaterkunst Breohts," Sinn und Form. 2. Sonderhoft. Berlini Rttten und Loening verlag, 1957. S. 159-177.

Bondz10, W1 theim. Die deutgche Spreche! Klelne Enzrklopldie In zuet Bunden. Hrsg. Erhara Agricola, Wolfgane Plo1soher und Helmut Protze. Leipz1g, VEB B1bllograph1sches Institut, 1969.

Bralut 1gam, Kurt. Bertozt Breont, Der gute Mensen ron Sezuan. Whohen: $\mathrm{B}$. Oldenbourg Vorlag, 1968.

Deblel. Gisela. "Das Prinzip der Verfremdung in der Sprachgestaltung Bertolt Breohta." D1seertation Bonn, 1960.

Froud, Anna. Slgmund Breud: Gesemmeite Werke. London, Imago Publ1 shing Co., Ltd., 1955. XIV, 438-439.

Hamburger, Kate. D1e Logik der D1chtung. Stuttgart, Ernst Klett Verlag, 1968.

Hartmann, N1colal. Aesthetik. Berlin, Walter De Gruyter \& Co., 1953.

Hauptmann, El1sabeth. Bertolt Brocht, Geasmelte Werke in 20 Banden. Werkausgabe EdItion. Frankrurt, suhrkamp Verlag, 1967.

Hultberg. Helge. Die Asthetigohen Ansoheuungen Bertolt Breohta. Munksgend und Kopenhagen! Munksgand International Booksellers and Publishers, Ltd., 1962.

Jagor, Gert. Die deutgohe Sprache: Kleine Enzrklopadie in zuel Bunden. Hrag. Erhard Agrioola, Wolfgang Fle1- 
scher und Helmut Protze. Lelpzig, VEB B1bliographisohes Inst1tut, 1969.

Jusowsk1, Josef. "Bertolt Breont und sein 'Guter Mensoh."" Sinn und Form. 2. Sonderheft. Berlins Bttten und Loening Vorlag, 1957. S. 204-213.

Kesting, Marianne. Das epische Theater. Stuttgart, W. Kohlhamer Verlag, 1969.

Klotz, Volker. Bertolt Brecht, Versuch Uber das Werk. 3. Aurl. Bad Homburg V.D.H. Beriln, Zuriohı Verlag Gohlen, 1967.

Klotz, Volker. "Interpretation des 'Guten Mensohen ron Sezuan." Materlalien zu Brechte "Der sute Mensoh von Sezuan." Hr\$8. Wermer Heoht. Ed1 tion Suhrkamp 247. 2. Auf 1. Frankfurt, Suhrkamp Verlag. 1969.

Le1s1, Ernst. Dex Wortinhalt, Selne Struktur 1m Deutsohen und Englisohen. 3. Aur 1. HeldeIberg, Quelle und Heyer, 1967 .

Porz1g, Walter. "Wesenhafte Bedeutungsbezlehungen." PBB, 58 (1934).

Porz1g, Walter. Wunder der Spreche. 4. Aur 1. Bern: Francke Verlag, 1957 .

Ralloke-Weiler, Kathe, Die Dramaturgle Breohts. Berlin! Eenschelverlag, 1966.

Wander, Karl Frledrioh Wilhelm. Deutsohes SpriohworterLexikon. 5 Bde. Leipzigi F,A. Brockhaus Verlag. 1867.

W11pert, Gero von. Saghworterbuoh der L1teratur. Stuttgart, Aifred Kroner Verlag, 1969. 